\title{
SVX4 User's Manual
}

L. Christofek, K. Hanagaki, J. Hoff, B. Kreiger, P. Rapidis, M. Garcia-Sciveres, M. Utes, M. Weber, R. Yarema, T. Zimmerman

Abstract: We present and describe the operation of the SVX4 chip. 


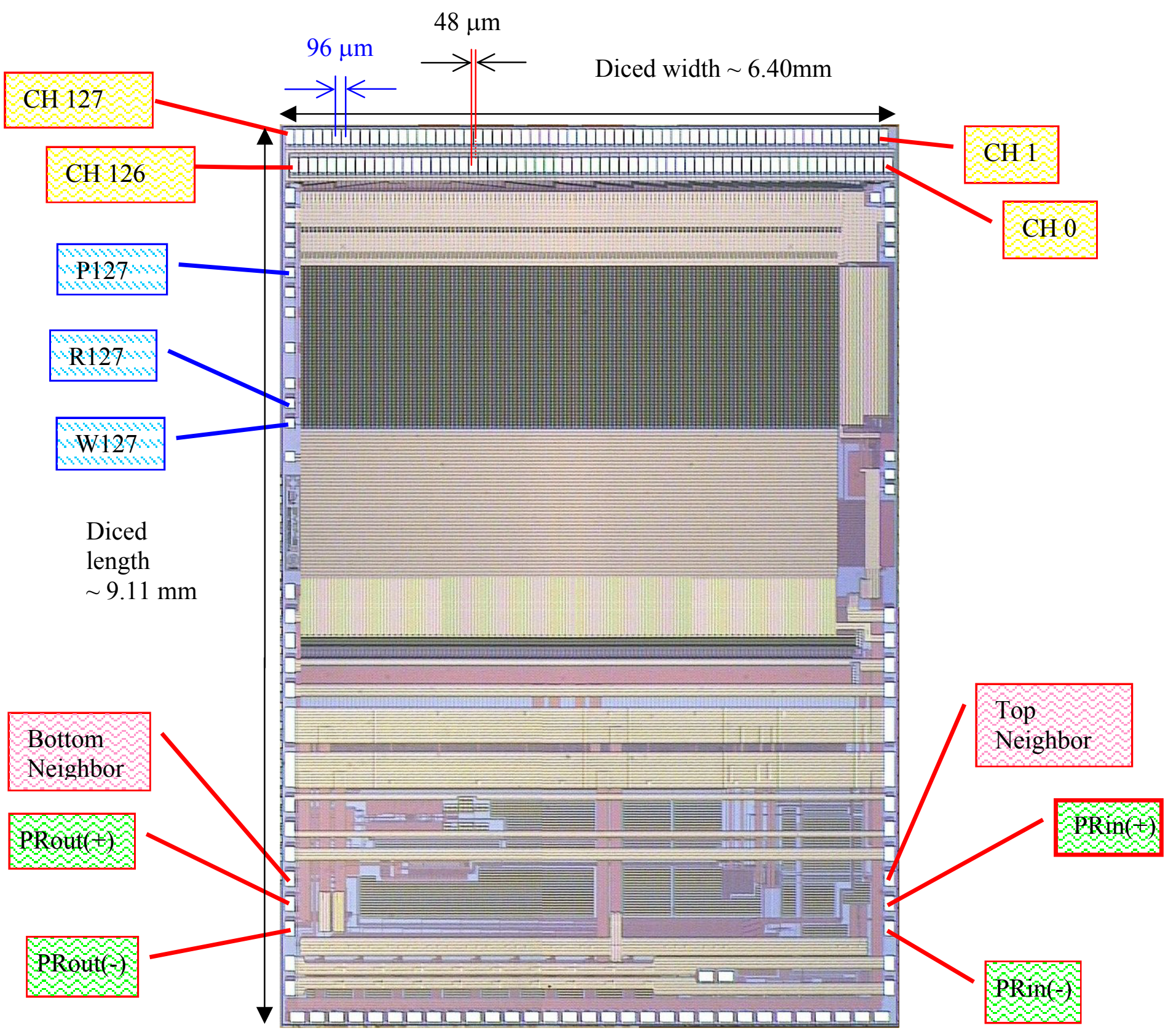

Figure 1 An actual picture of the SVX4 chip. The bottom of the picture is the back-end of the chip and the upper half is the front-end. The 128 input pads can be seen at the top of the picture. The Priority in/out, and Top/Bottom Neighbor are indicated at the bottom of the picture. The three buffered diagnostic analog probe points of the last channel (127) are also shown on the left. This chip is fabricated with the 0.25 micron TSMC process on $300-m i c r o n$ thick silicon. 


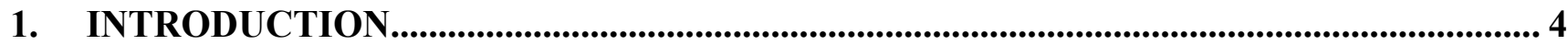

1.1 SILICON STRIP DETECTORS FOR CDF AND D0 FOR RUN IIB ........................................... 4

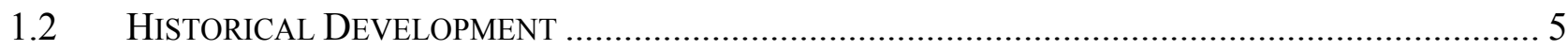

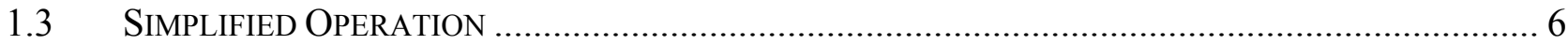

2 FUNCTIONAL DESCRIPTION ............................................................................................. 10

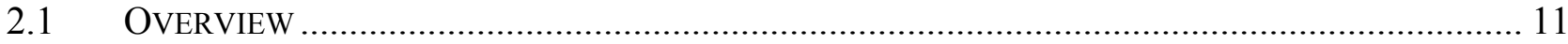

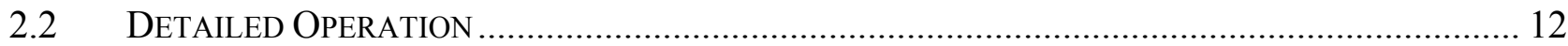

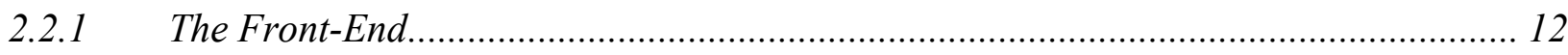

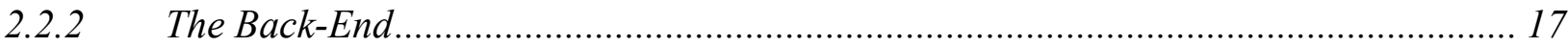

3 THE DIFFERENCE BETWEEN DO AND CDF MODES ..................................................... 21

4 INITIALIZATION BIT STREAM ...................................................................................... 23

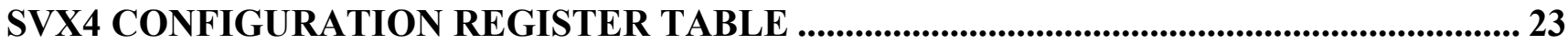

4.1 SVX4 CONFIGURATION REGISTER EXPLANATION ........................................................... 24

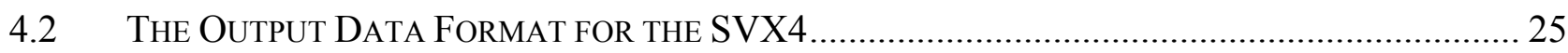

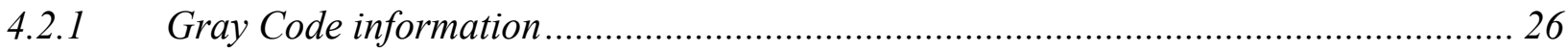

5 MECHANICAL AND ELECTRICAL SPECIFICATIONS …................................................ 28

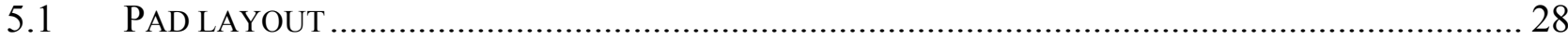

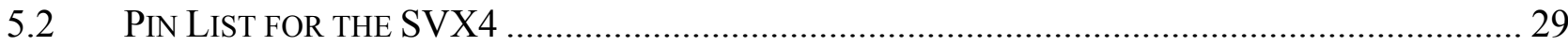

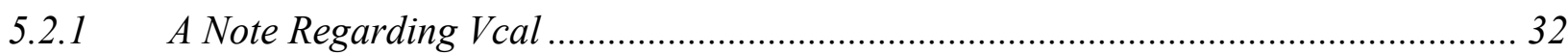

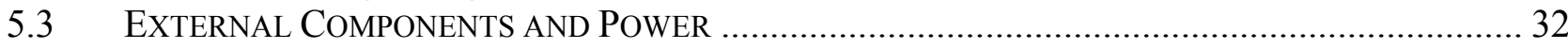

6 OPERATING THE CHIP ........................................................................................................ 34

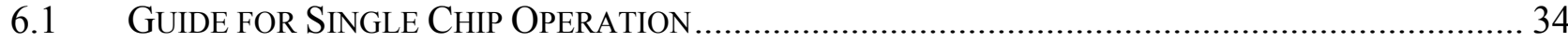

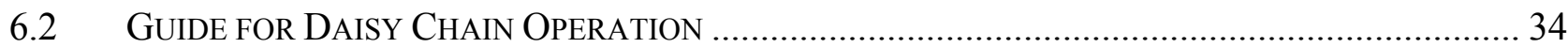

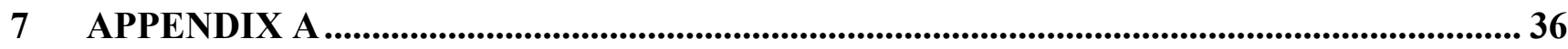

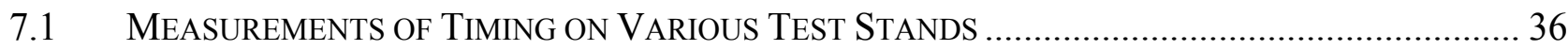

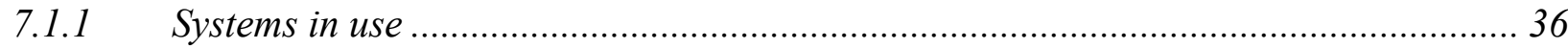

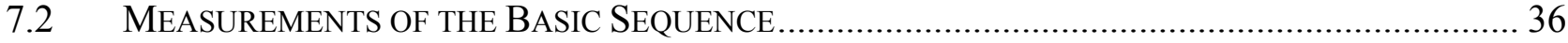

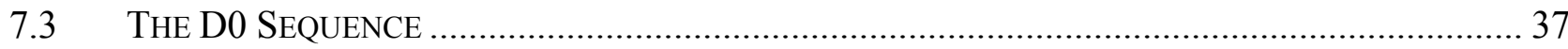

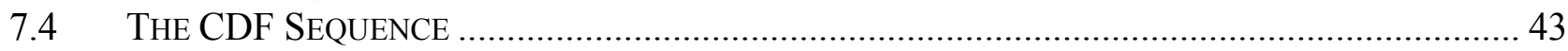

8 APPENDIX B -THE SVX4 SPECIFICATIONS (ORIGINAL LIST) .................................... 48

9 APPENDIX C - DECIMAL/GRAY TABLES.............................................................................. 53 


\section{Introduction}

The SVX4 is a custom 128-channel analog to digital converter chip used by D $\varnothing$ and CDF in Run IIb to read out their respective silicon strip detectors. Each channel consists of an integrator (Front-End device, or FE) and a digitize/readout section (Back-End device, or BE). The input to each channel is sampled and temporarily stored in its own storage capacitor. Upon receiving a trigger signal, the relevant pipeline cell is reserved. Subsequent signals cause reserved cells to be digitized by a 128 parallel channel Wilkinson type 8-bit ADC, and then readout in byte-serial mode with optional zero suppression (sparsification). Salient features include (1) operation in either D $\varnothing$ mode or CDF mode (CDF mode features "dead timeless operation" or continued acquisition during digitization and readout) with an additional mixed mode of operation, (2) adjustable, loadable control parameters, including the integrator bandwidth and ADC polarity (only one input charge polarity will be used for Run IIb, but this feature remains for diagnostic purposes), (3) sparsified readout with nearest neighbor logic, (4) built-in charge injection with the ability for external voltage overriding for testing and calibration, and (5) a channel mask that is used for either charge injection or for masking of channels with excessive DC current input during chip operation. This document is meant to familiarize the user with the functionality of the SVX4 and goes on to include specifications, pin outs, timings and electrical information. Additional information on the SVX4 can be found in Ref [1].

\subsection{Silicon Strip Detectors for CDF and DO for Run IIb}

Both D $\varnothing$ and CDF for Run IIb have opted to use silicon strip detectors for their vertex detectors that are (1) single sided p-implant on n-type material, (2) 300 micrometers thick, (3) have typically a strip pitch of $\sim 50$ micrometers, (4) are resistively biased with bias resistors that are on the detector itself ( $\mathrm{R}$ bias $\sim 2$ megaohms), (5) are capacitively coupled to the readout chips with on board capacitors (that are formed by a dielectric/aluminum strip structure deposited on top of the p-implant strips, Ccoupling $\sim 25 \mathrm{pF}$ ), and (6) are biased to full depletion by applying a positive potential to the $\mathrm{n}$-side (ohmic, backplane) side of the detector and thus have the p-implant (junction) side at ground potential.

A simplified schematic is shown in Figure 2. Ionizing particles traversing the depletion region of this backwards biased diode liberate electron-hole pairs and thus cause a short burst of charge to appear at the input capacitor. The first stage of the SVX4 chip is an integrating amplifier that collects the charge during a well defined period of time, which in the case of the two Tevatron experiments is roughly equal to the time between beam bunch crossings, and presents this integrated charge to a charge storing pipeline and ultimately to the digitizing circuit for digitization. 


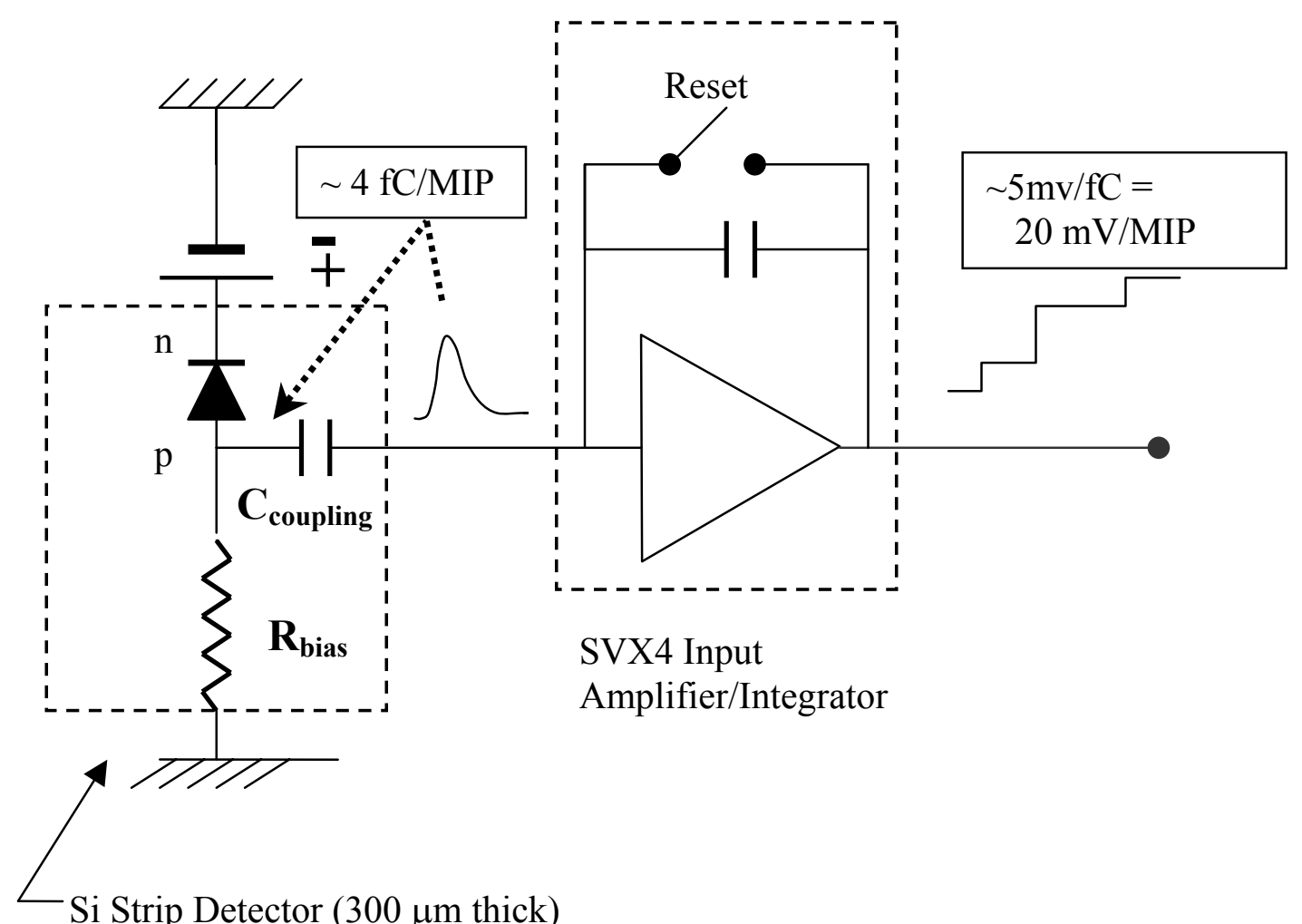

Figure 2 Block diagram of a Silicon Strip Detector and the front end of the SVX4 chip. The biasing scheme and polarities of the signals for the Run IIb vertex detectors of both CDF and D0 are indicated.

For completeness, it should be noted that for normal incidence of a Minimum Ionizing Particle (MIP) for a silicon detector thickness of 300 microns the expected signal consists approximately of 22000 electron-hole pairs, which corresponds to $3.5 \mathrm{fC}$. (Note that $1 \mathrm{fC}=6241 \mathrm{e}$ 's, $1 \mathrm{e}=1.602 \times 10^{-4} \mathrm{fC}$ ).

\subsection{Historical Development}

In the late 1980's, several versions of a fully custom chip called the SVX were built and tested [2,3]. As part of the Run IIa upgrade for D $\varnothing$ the SVX2 and for CDF the SVX3 were designed to meet the needs of the experiments by a collaboration of engineers at Fermilab and Lawrence Berkeley Laboratory $[4,5,6,7]$. Requirements dictated that the devices should be capable of operating at an interaction rate as fast as $132 \mathrm{nsec}$, that it have optimal performance for detector capacitances between 10 and $35 \mathrm{pF}$, and that it have an analog pipeline with a maximum delay of about $4 \mu$ sec to allow time to form a trigger signal. 
For the SVX2 chip used by D0, when a trigger signal is received, data acquisition stops until the chip is completely read out. As plans for Run IIa evolved, dead time because an issue for CDF and the development of the SVX3 with a dead timeless feature ensued. The SVX4, the successor design to the SVX31, is a chip that can be used in either dead timeless mode (CDF) or in an arrested mode $(\mathrm{D} \varnothing)$. A large effort and several iterations proved necessary to overcome digital-analog coupling issues seen in dead timeless operation for previous versions of the SVX4 prototype.

The main features and specifications of the SVX4 are given below:

1. 128 channels per chip,

2. Maximum interaction rate equal to $132 \mathrm{nsecs}$,

3. Optimized for capacitive loads from 10-35 pF,

4. Channel mask with dual functionality: used for either charge injection or masking channels with excessive DC current from the detector,

5. Choice of operation in either $\mathrm{D} \varnothing$ or CDF mode using an external pad as selector,

6. Selectable input bandwidth,

7. Double correlated sampling (see section 2.2.1),

8. Large dynamic range on input integrator to minimize dead time due to pre-amplifier resets,

9. Programmable analog pipeline ( 47 cells, 42 cells maximum depth for pipeline, 4 cells for trigger buffer, 1 cell for write amplifier pedestal),

10. Digitization of analog signals with up to 8 bits of resolution using a modified Wilkinson type ADC,

11. Dynamic (real time) pedestal subtraction,

12. Data sparsification (zero suppression),

13. Neighboring channel readout selection (cluster readout),

14. Low noise $(\mathrm{S} / \mathrm{N}=10: 1$ to $20: 1$ for input capacitances from $35 \mathrm{pF}$ to $10 \mathrm{pF}$ for an input charge equivalent to $1 \mathrm{MIP}=4 \mathrm{fC})$,

15. Low power consumption (approximately $3 \mathrm{~mW} /$ channel) to minimize the cooling requirements,

16. Operation with a single voltage source (even though two separate decoupled supplies for the analog and the digital portions of the chip may be required),

17. Operation compatible with single-sided AC coupled silicon strip detectors,

18. Ability to inject charge for testing and calibration in each channel,

19. Daisy chain operation capability,

20. Parallel bus data readout,

21. Integral Data Valid strobe signal in the data bus (OBDV),

22. Can be implemented in the TSMC (Taiwan Semiconductor Manufacturing Company) 0.25 micron process that is inherently a radiation hard process.

This document is arranged as follows: Section 2 gives a detailed description of the chip's operation, including timing diagrams, Section 3 defines the initialization bits in detail. The electrical specifications are given in Section 4 and Section 5 describes how to connect and mount chips. The appendices compare settings and measurements of the chip on several different test stands with those from the prototype DAQ system.

\subsection{Simplified Operation}

The SVX4 is comprised of 128 channels of identical electronics along with additional circuitry that is common to all channels. Figure 3 shows a simplified diagram of one of the identical channels of electronics and some of the common circuitry. Charge is received from the silicon strip detector via 
the input bond wire and integrated on a $220 \mathrm{fF}$ feedback capacitor, $\mathrm{Cf}$, which sets the DC gain of the input amplifier to be $5 \mathrm{mV} / \mathrm{fC}$. In addition to the detector input, a separate $25 \mathrm{fF}$ test input capacitor, $\mathrm{Ct}$, is connected to each integrator via a programmable switch. The capacitor allows each channel to be pulsed independently (synchronously with with a common control pulse) to study channel operation or provide simulated events to the SVX4 in the data acquisition system. The AC response of the front end electronics is determined primarily by the integrator response (there is no shaper). For different interaction times and input capacitances, the bandwidth of the preamplifier is adjusted by means of control registers to provide the optimal preamplifier output rise time and hence minimum noise.

The output of the preamplifier (integrator) feeds the analog pipeline which has a length of 46 cells, a number determined by the minimum interaction time and maximum required time delay. The pipeline has a fixed voltage gain of three determined by the ratio of the value of the input coupling capacitor, $\mathrm{Cc}$, and the storage capacitors, $\mathrm{Cn}$. The effective depth of the pipeline is the same for all channels and can be set via digital control to have any value from 1 to 42 samples. The pipeline operates by sequentially sampling the output of the preamplifier on one of 46 storage capacitors. After each interaction period, switch Sd in the pipeline resets the next sampling capacitor causing the output of the preamplifier to be stored on the coupling capacitor, $\mathrm{Cc}$, and thus performing a double correlated sample on the preamplifier output (this way the integrator baseline does not matter, only the change in level during a given beam crossing is stored in the pipeline). The integrator output is allowed to build until it can be reset by switch $\mathrm{Sa}$ at a convenient time as shown in Figure 4. 


\section{Preamplifier Analog Pipeline Comparator Digital Functions}

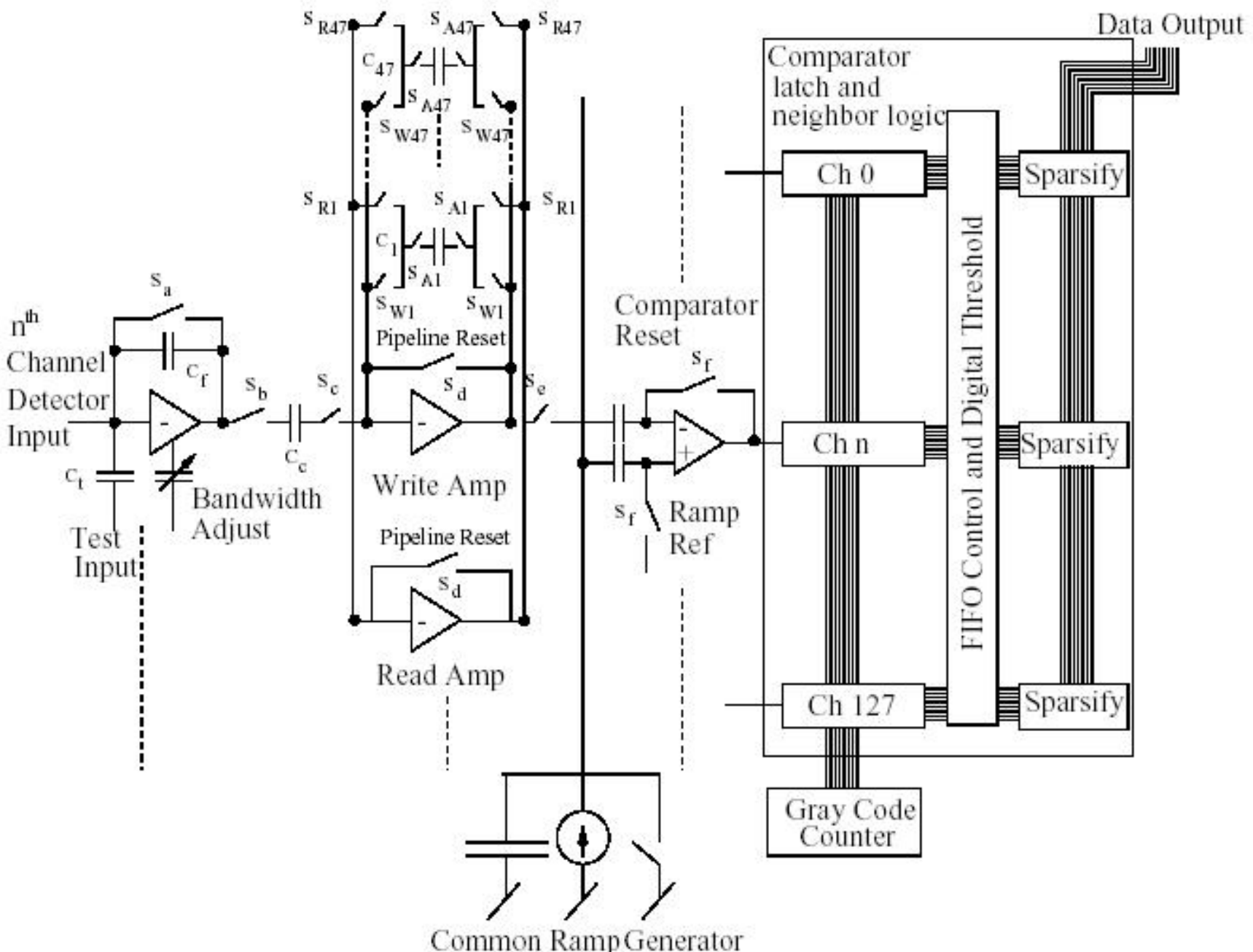

Figure 3 Simplified single channel block diagram.

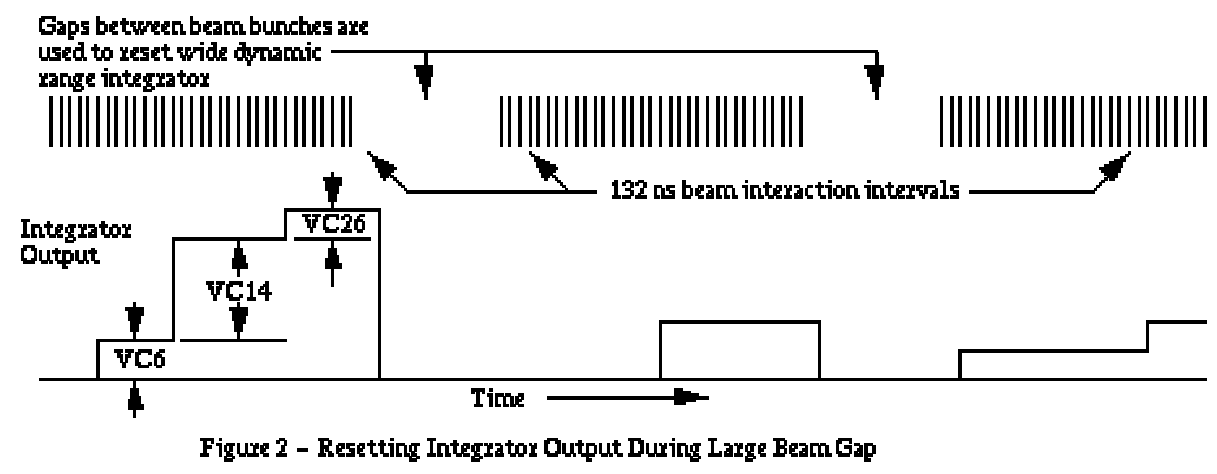

Figure 4 Resetting integrator output during large beam gaps. 
The voltage change indicated by VC6, VC14, and VC26 (which is indicative of charge injected at the input of the SVX at these three times) is stored on a sample capacitor for subsequent readout. Charge injection from opening $\mathrm{Sd}$ and the sample switches is stored on the sample capacitor along with the desired signal. These charge injection effects are compensated during the pipeline readout using a $47^{\text {th }}$ pipeline cell reserved exclusively for this purpose. Resetting a storage capacitor can be done in $20 \mathrm{~ns}$. However, resetting the preamplifier requires a settling time of the order of $200 \mathrm{~ns}$ and is therefore reset during the major beam gaps in the main ring beam structure or at other times that do not interfere with normal data taking. The dynamic range of the preamplifier is $200 \mathrm{fC}$.

Readout of the SVX4 begins when a Level 1 Accept control signal (derived from the system trigger) is sent to the chip. Depending on which mode the chip is configured, two things can occur: 1) in D $\varnothing$ mode, pipeline acquisition should stop and pipeline readout of the appropriate storage capacitor should begin and 2) in CDF mode, pipeline acquisition continues and the appropriate pipeline is stored in a secondary pipeline where it awaits the readout and digitization process.

When the proper control signals are sent to the chip for pipeline read out, a pedestal correction is performed on the stored signal in the pipeline to correct for variations in switch charge injection and other errors; this is accomplished by subtracting the value stored on the $47^{\text {th }}$ pipeline cell from the stored signal. The output of each pipeline feeds a Wilkinson type 8 bit ADC. The ADC is formed by a separate analog comparator, analog delay (which is used for dynamical pedestal subtraction), a counter latch for each channel and common ramp generator and Gray Code counter which is used for all of the channels. Digital conversion is initiated by activating the analog comparator for each channel and then starting the ramp generator and Gray Code counter. The ramp is applied to the analog comparator along with the input signal to be digitized. When the comparator output changes, the counter latch is set (after passing through the analog delay) which stores the output of the Gray Code counter for that channel. The number stored in the digital latch is a measure of the amount of charge that was integrated by the preamplifier from a given interaction. With data sparsification on, when the number in the Gray Code latch exceeds a programmed threshold setting, that channel is considered to have a hit and it is tagged for readout.

The SVX4 is designed to work with AC coupled single sided detectors, and is optimized for current pulses of the polarity shown in Figure 2, which we call positive polarity. It is able to accept negative polarity input signals but with a much more limited input range (see Ref [8]). The functionality discussed below is only used for diagnostic purposes. Several signal inversions take place inside the SVX4 chip. The output of the preamplifier is inverted as shown in Figure 1 (e.g. the output signal level is negative going for positive input current and positive going for negative input current). The pipeline inverts the preamplifier signal. The technique used to read out the pipeline causes a third signal inversion to occur. Thus during pipeline readout which occurs prior to digitization, the signal level to the analog comparator is negative going for positive input current or positive going for negative input current.

For proper operation, externally programmed polarity signals are used to choose either positive or negative input operation for the chip. Three bits (Pipeline Readout Order, Ramp Polarity, and Comparator Polarity) are provided for maximum flexibility to set levels inside the chip and establish the proper operation. The polarity signals perform three different functions inside the SVX4 chip. First, Pipeline Readout Order selects whether the signal presented to the ADC is formed by either subtracting the pedestal (stored on pipeline cell 47) from the signal (if the bit set to 1) or by subtracting the signal from the pedestal (bit set to 0). Second, the Ramp Polarity bit controls the 
direction (positive (0) or negative (1)) of the ramp generator to correspond to the polarity of the input signal. The third polarity bit, Comparator Polarity, is used to either pass (0) or invert (1) the comparator output so that the signal delivered to the following logic has the same meaning for both positive and negative current input signals. The usual operation for both CDF and D $\varnothing$ has all three polarity bits set to 0 . See Figure 10 in this document.

As shown in Figure 1, the analog comparator feeds a latch and neighbor hit logic. The SVX4 data readout can take one of three different forms depending on the status of two control bits called Read Neighbors and Read All in the neighbor hit logic. If both of these bits are low, the channels to be read out are only those channels (i.e. hit channels) whose digitized outputs exceed the threshold level that was digitally downloaded. If the Read Neighbor bit is set high, then hit channels and the channel immediately on each side of the hit channel are also read out. When chips are daisy chained together, neighbor information is passed from one chip to another so that if an end channel is hit, a neighbor channel on the adjacent chip is read out. The readout of neighbor channel amplitudes allows for interpolation to obtain higher hit location accuracy. Under some situations such as testing, all channels on a chip can be read out regardless of signal level by setting the Read All bit high.

The hit threshold level for an SVX4 chip is set digitally and is the same for all channels on that chip. Normally the threshold is set at some fraction of a MIP that results in a relatively coarse threshold resolution (e. g. 2000 e). To overcome this problem, control of the A/D ramp start voltage is provided which allows fine tuning of the noise hit rate. An internal adjustment of the ramp start voltage (RAMP-PED) effectively allows the threshold to be adjusted with 400 e resolution.

The output of the neighbor logic circuit from all the channels forms an ordered array of the channels to be read out. Before the chip is read out, the address and data for each channel to be read is stacked in a FIFO that uses a token passing system for readout. When readout does begin, channels are read out sequentially beginning with the lowest address channel. The geographical location of channel 0 is indicated on Figure 1. Since this token passing scheme takes some time, readout of a chip in sparse mode with only a few channels at the high end (i.e. near channel 127) having a valid signal may fail for the anticipated readout rate giving a double readout [9]; for that reason a Read Channel 63 bit may be set to force readout at this intermediate point and allow for the token ring passing to 'catch-up'. A Read Channel 127 bit is also included for diagnostic purposes.

Control of the SVX4 and data readout is handled by digital and bias pads in the I/O section of the right hand side of the chip. There are three pads called FEMODE, BEMODE, and CHMODE that are used to select one of the four possible operating cycles (Initialize, Acquire, Digitize, and Readout) for the SVX4 when in CDF mode. Sixteen pads, called BUS0-7 and BUS0-7 bar, are used to output address and data information from the SVXII during the Readout Mode. The same sixteen pads are used for real time control of internal switches in the other three operating modes (only for $\mathrm{D} \varnothing$ operation, CDF operation was described earlier). For these three modes, the last information on the pads prior to a mode change is held on internal latches before switching to the next mode. Two other pins, Bottom Neighbor and Top Neighbor, are used to communicate with adjacent chips on neighbor readout. Priority In and Priority Out are used to communicate with adjacent chips. These two Priority pins carry different information for each of the four different operating modes.

\section{Functional Description}

In this Section, the function of the SVX4 is described. Section 2.1 gives a brief overview, Section 2.2 describes the operation of the chip in detail, and Section 2.3 gives detailed timing diagrams. Items in 
the initialization bit stream are described as they relate to operation; a concise list is deferred to Section 3. Section 2.4 gives the physical layout of the chip, including tables of all the input and output pads. Section 2.5 describes the format for the data output and explains the Gray coding scheme used.

\subsection{Overview}

The SVX4 consists of 128 identical channels. Each channel has two parts, a Front-End and a BackEnd. The Front-End contains the integrator and storage pipeline. The Back-End contains the ADC for digitization and the readout logic and drivers. The major cycles of operation for these parts are Initialization (both), Acquisition (Front-End), Digitization (Back-End), and Readout (Back-End). The initialization cycle usually is performed once, followed by repeated data acquisition, digitization, and readout cycles. The Acquisition cycle occurs simultaneously with the Digitize and Readout cycles in CDF mode, but operates exclusively in $\mathrm{D} \varnothing$ mode. Three input signals, FEMODE, BEMODE, and CHMODE are used to change the modes as summarized in Table 1.

\begin{tabular}{|c|c|c|c|}
\hline FE Mode & BE Mode & $\begin{array}{c}\text { chip state } \\
\text { when in CDF mode }\end{array}$ & $\begin{array}{c}\text { chip state } \\
\text { when in D0 mode }\end{array}$ \\
\hline 0 & 0 & Initialize & Initialize \\
\hline 0 & 1 & -- & Acquire \\
\hline 1 & 0 & Acquire \& Readout & Readout \\
\hline 1 & 1 & Acquire \& Digitize & Digitize \\
\hline
\end{tabular}

Table 1 Table showing the various states of the chip in either CDF mode or $D \varnothing$ mode depending on the various conditions of the mode levels (note that these mode levels appear as either inputs on dedicated pads (in CDF mode) or on Bus lines (in D0mode)).

To change the state of the front or back-end, the mode bits are changed, and then the CHMODE pad is pulsed high to complete the transition to the new state of operation. To provide noise immunity and stable operation, mode signals are internally latched on a transparent latch controlled by CHMODE. Thus, if CHMODE is low, changes in the mode signals do not change the internal state of the chip; if CHMODE is high, the internal state of the chip is determined by the mode signals. Timing specifications and the appropriate levels for FEMODE and BEMODE for each of the three cycles (initialization, acquire, and readout) are given in Section 2.3.

It is also important to realize that depending on which mode (D0 or CDF) has been configured, the bus lines will operate as control for the ADC only (CDF mode) or will operate as control lines for operation in acquire or digitize (D $\varnothing$ mode). The function of the control lines is shown in Table 2 . Depending on the mode of the chip, the bus lines could have different functions. 


\begin{tabular}{|c|c|c|}
\hline $\begin{array}{c}\text { Pin name, } \\
\text { Readout } \\
\text { mode } \\
\text { function }\end{array}$ & $\begin{array}{c}\text { Digitize mode } \\
\text { function }\end{array}$ & $\begin{array}{c}\text { Acquire } \\
\text { mode } \\
\text { function in } \\
\text { Do mode } \\
\text { only }\end{array}$ \\
\hline Bus0 & Comp_rst & -- \\
\hline Bus1 & Ramp_rst & -- \\
\hline Bus2 & -- & PRD2 \\
\hline Bus3 & Rref_sel & -- \\
\hline Bus4 & -- & PARST \\
\hline Bus5 & -- & L1A \\
\hline Bus6 & -- & PRD1 \\
\hline Bus7 & -- & CalSR \\
\hline
\end{tabular}

Table 2 Bus pin multiplexing table. CALSR is ORed with the CALSR pad in the Acquire cycle and ORed with WrSEU in the Initialize cycle.

\subsection{Detailed Operation}

\subsubsection{The Front-End}

The SVX4 front end was designed at Fermilab and mates with the SVX4 back end, designed at LBL, to produce a complete SVX4 128 channel silicon detector readout chip. The front end contains 128 identical channels of integrating charge preamp and a 46 cell analog pipeline that is cycled by the beam crossing clock. Hit cells are temporarily removed from the pipeline for readout to the back end, where the data is digitized, sparsified, and read out. SVX4 is "dead timeless," so that front end signal acquisition can continue uninterrupted while back end digitization and readout is occurring. Operation of the front end requires only a $2.5 \mathrm{~V}$ supply, a front end clock, and a few digital control lines. The front end has two modes of operation: Initialize and Acquire.

In Initialize mode, the front end clock signal (FEClk) is routed to control a 148 bit shift register, which is downloaded with program bits. 20 of these bits set programmable parameters such as trigger delay, bandwidth, bias current, etc. The remaining 128 bits form a mask register that is used to selectively enable or disable reception of a calibration test charge to each of the 128 preamp inputs. The serial program bit stream line, Srin, actually comes from the back end chip which also has a programmable register and is a copy of the level presented on PRIN. The serial data is clocked into the registers on the rising edge of FEClk. After downloading of the shift register is complete, application of a strobe pulse (via the CalStrobe control line) transfers the 20 programmable parameter bits to an SEU tolerant shadow register. The strobe also resets the pipeline cell position 0 (i.e. to Cell0). Initialization must be performed after power up and before acquisition begins. Although theoretically not necessary, it may be desirable to periodically repeat initialization to insure that the chip remains in a known operating condition. 


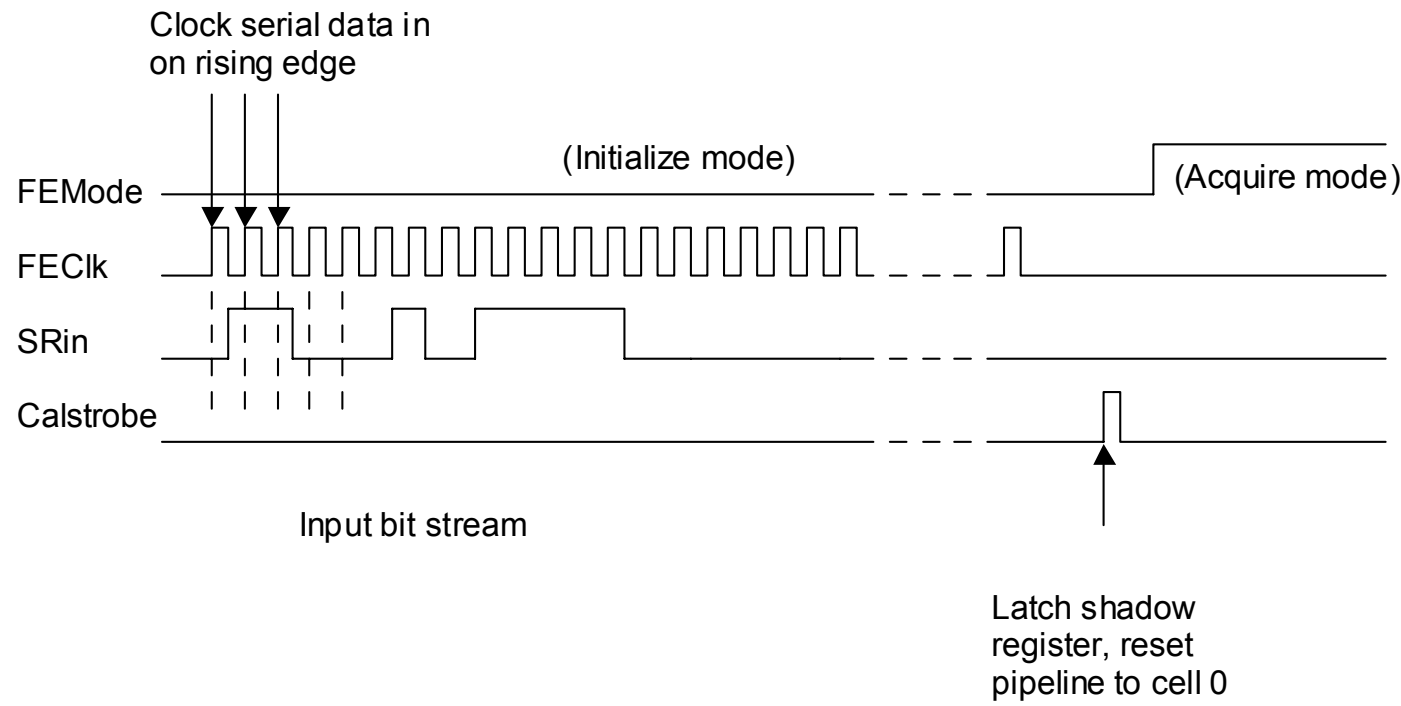

Figure 5 Timing diagram for the initialization of the SVX4.

In Acquire mode (see Fig. 5), the front end clock (FEClk) is routed to the analog pipeline and is used to advance the 46 cells in round robin (i.e. circular) fashion at the beam crossing rate. At each of the 128 channel inputs, an integrating charge preamp accepts a positive input charge from the detector, and the preamp output feeds the pipeline. The system charge transfer gain is $15 \mathrm{mV} / \mathrm{fC}$ (this is the transfer from input to the SVX4 to the input to the ADC). As the pipeline cells are advanced with the front end clock, they perform correlated double samples on the preamp output, as will be described. A given cell is reset while the front end clock is high, takes a first sample of the preamp output when the clock goes low, and takes the second sample when the clock goes back high, which also advances the pipeline to the next cell. The voltage difference between the two samples, representing the preamp charge integrated during that time, is thus stored in the cell. The duty cycle of the clock obviously controls the amount of time spent resetting and acquiring on a cell. Typically, the front end clock should have a low duty cycle so that only a small portion of the clock cycle time (minimum 20 ns) is spent resetting, and most is used for acquiring the preamp output. This is desirable since the slower the preamp risetime, the lower is its series noise. We show an example of the risetime as a function of the bandwidth setting for two different values of the external capacitors in Table 3 .

\section{Measured PreAmp risetimes (in ns, $10 \%$ to $90 \%$ ) for several BW settings vs. Load Capacitance}

\begin{tabular}{|c||c|c|c|c|c|c|c|c|c|}
\hline BW & 0 & 1 & 2 & 3 & 4 & 7 & 8 & 11 & 15 \\
\hline $10 \mathrm{pF}$ & 20 & 23 & 27 & 32 & 35 & 46 & 48 & 58 & 64 \\
\hline $33 \mathrm{pF}$ & 38 & 45 & 53 & & 65 & & 82 & & \\
\hline
\end{tabular}

Table 3 The risetime as a function of the bandwidth setting for two different values of the external capacitance. 
The dynamic range of the preamp (200 fC) is larger than the dynamic range of the pipeline (40 fC), so that many signal charges can be integrated and sampled without saturating the preamp. However, the preamp must periodically be reset via an external control line (PreampReset) in order to prevent eventual saturation. PreampReset is active high, with a minimum required width of $80 \mathrm{~ns}$ to achieve complete reset. It is typically performed during beam gaps in order to avoid incurring any deadtime. The timing of PreampReset is not critical, but after reset, one beam crossing time ( 132 nsec) should be allowed for the preamps to settle before inputs can be accurately acquired.

The Level 1 Accept (L1A) control input is used to remove a "hit" cell from the pipeline, with a delay from 1 to 42 beam crossings, and temporarily stores it in a FIFO so that it is queued for readout to the back end. The delay is determined by the value programmed in the shift register during Initialize mode. L1A is normally high during acquisition, and pulsed low to store a cell. L1A must go low and return high between front end clocks, i.e., while FEClk is low. Up to four cells can be stored in the FIFO and queued for readout. If four cells are stored, additional L1As are simply ignored.

A special pipeline cell, the "pedestal cell," is reserved for acquiring a pedestal only. It is used during readout along with a stored cell. The back end essentially digitizes the difference between the hit cell and the pedestal cell. The pedestal cell is not part of the normal round robin of acquisition cells, and must be explicitly refreshed periodically. This is one of the functions of the PR2 control line. If PR2 is high when FEClk transitions from low to high, then normal acquisition is inhibited for that clock cycle. The normally intended pipeline acquisition cell is skipped over and the pedestal cell instead is placed in the pipeline for acquisition of the pedestal. Thus one cycle of dead time is incurred by refreshing the pedestal cell. If this is done during a beam gap, dead time can be avoided. The timing diagram for data acquisition and preamp reset is shown in Figure 6.

Operation of SVX4 is "dead timeless," so that the readout and digitization process can occur in parallel with normal acquisition. Front end cell readout is accomplished by asserting the PR1 control line in conjunction with FEClk (which continues to control normal acquisition). If PR1 is high at the low to high transition of FEClk (PR1 should then subsequently be lowered), the pedestal cell readout is then initiated. The read amp is reset during the first clock cycle, then the pedestal cell is held in the read amp at the start of the second clock cycle. The read amp output feeds the back end, which uses the pedestal voltage to autozero the ADC. When PR1 is raised a second time, the next FEClk low to high transition removes the pedestal cap from the read amp and initiates readout of the stored hit cell, which is read out in a manner similar to the pedestal cell. The hit cell voltage can then be digitized by the back end. If desired, the effective signal polarity which is digitized can be reversed by setting to 1 the Pipeline Readout Order parameter bit in Initialize mode. This reverses the readout order to (signal - pedestal) instead of (pedestal - signal). After digitization is complete, the readout cell needs to be removed from the FIFO and placed back into the pipeline. This is accomplished by doing a PR2, which has the dual function of digitally restoring the cell to the pipeline and of retaking the analog pedestal on the pedestal cell capacitor. The timing diagram for pipeline readout is shown in Figure 7. 


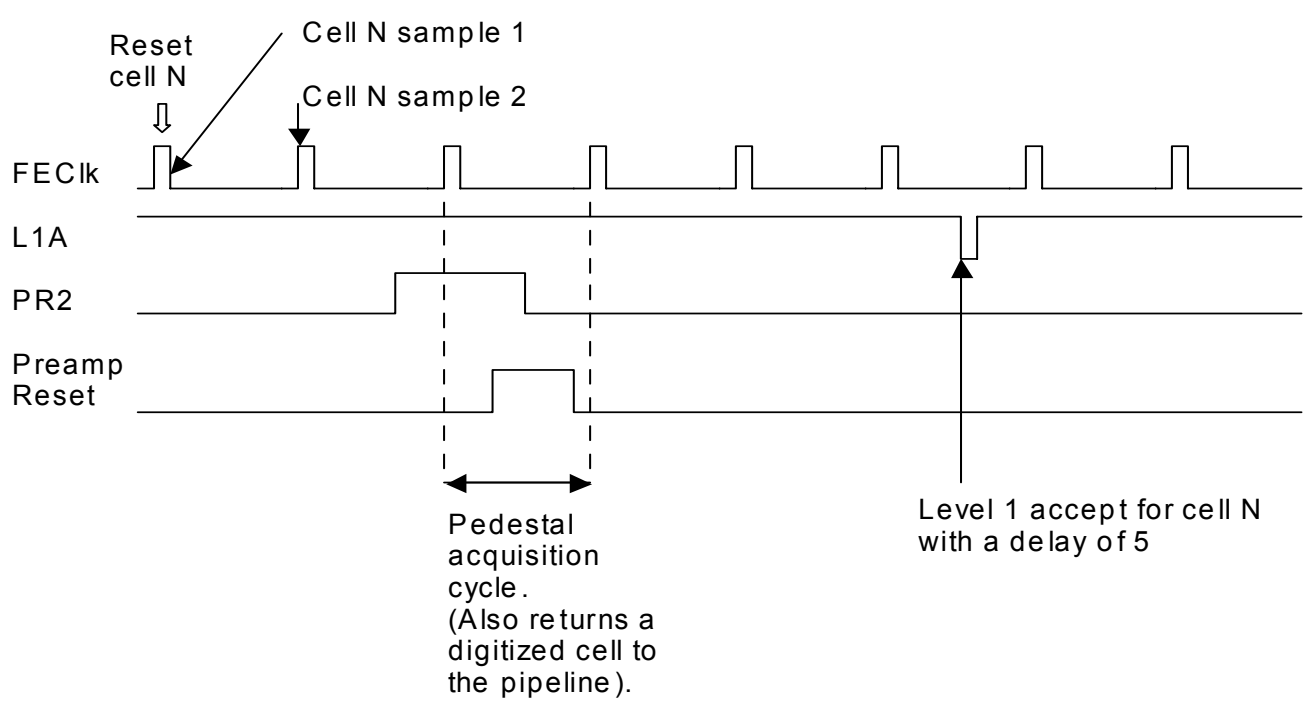

PreampReset shown during pedestal acquisition cycle, but this can occur a ny time.

Figure 6 Timing diagram for the acquire cycle of the SVX4.

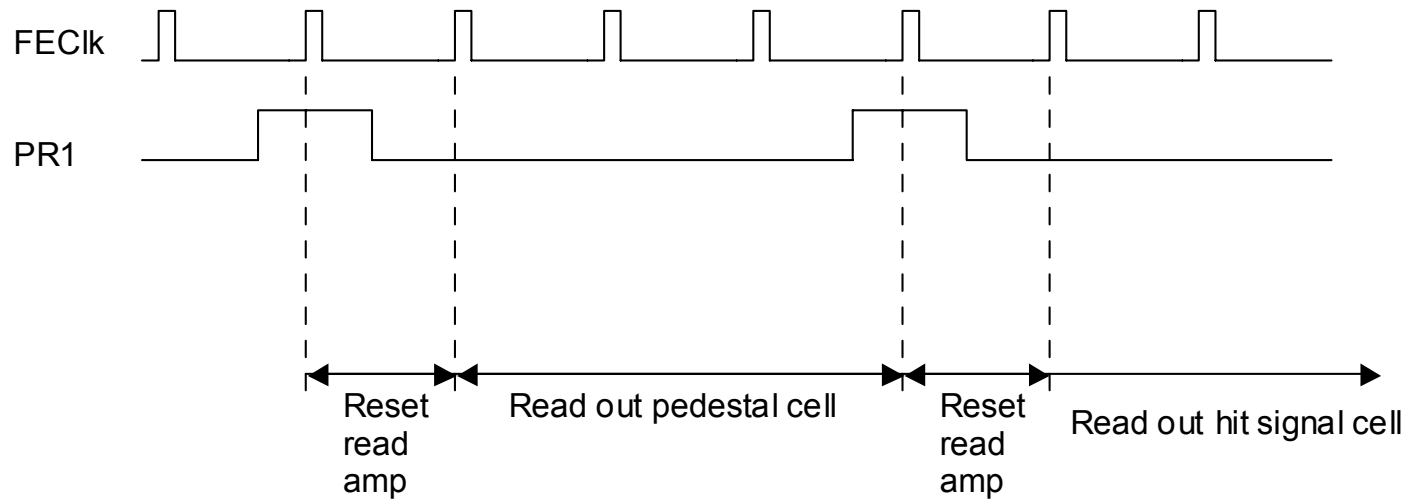

Figure 7 Timing diagram for the pipeline readout of the SVX4.

In order to facilitate testing, a small charge injection capacitor ( $25 \mathrm{fF}$ ) can be switched in from each preamp input to a common bus line. A 128 bit programmable channel register (downloaded in the Initialize mode) can function as a mask register, and determines whether or not an injection capacitor is switched in for each channel. When in the Acquire mode, the common bus voltage is determined by the state of the CalStrobe control line. When CalStrobe is low, the common bus is grounded. When CalStrobe goes high, the common bus is connected to the VCAL pad. Thus, raising CalStrobe injects a charge of magnitude $(\mathrm{VCAL})(25 \mathrm{fF})$ to each channel that has a mask setting of 0 . 
Usually it is desirable for all channels on a chip to be functional. However, sometimes "black hole" effects (usually due to pin-hole shorts of the coupling capacitors of the silicon strip detectors) are present in detectors, which result in a DC current being applied to a preamp input. This can affect neighboring channels by turning on input diode protection circuits, which can activate parasitic current paths. Therefore, a provision has been included which allows a selected channel's preamp reset to be held high, which harmlessly sinks any positive input current to ground without affecting any other channels. Setting the programmable Mask/Disable bit during Initialize enables this feature. If Mask/Enable is high, then the 128 bit channel register is used not as a charge injection mask register, but as a channel disable register. Any channel that has its mask bit set high will have its preamp reset held always high.

Powering the SVX4 Front End is very straightforward. An analog power supply, AVDD, of 2.5V must be provided for the preamp and the analog sections of the pipeline. This supply is bypassed on chip with an integrated $0.012 \mathrm{uF}$ capacitor. Best dead timeless performance is obtained if an external $0.1 \mathrm{uF}$ bypass capacitor is added close to the chip (within an inch or so). The front end analog ground is NOT supplied through a pad, but through the low resistance back side of the die. Thus, the die must be connectively attached to a ground plane. A digital supply (DVDD and DGND) is required to drive the pipeline digital logic. This supply is not derived from front-end pads, but is routed in from the back end chip digital supply. For optimal dead timeless operation, AVDD and DVDD should come from two physically separate power supplies. If, however, front end acquisition will not be occurring simultaneously with back end digitization and readout, it may be possible to derive AVDD and DVDD from the same external power supply.

There is an internal master bias circuit on the front-end chip, which supplies the bias reference for both preamp and pipeline. Preamp and pipeline bias currents can be adjusted via programmable shift register bits. The on-chip bias reference voltage is connected to the Bias pad. Under normal conditions, no external bias current reference needs to be provided. Since the bias circuit is referenced to AVDD, an on-chip Bias to AVDD bypass capacitor is included. An external bypass capacitor from Bias to ground can be provided in order to improve the integrator Power Supply Rejection Ratio (PSSR). The optimal value of this bypass will depend on the value of the integrator input capacitance to ground (not to neighbor channels).

Two preamp diagnostic bias pads are included on the prototype so that they can be forced if necessary. Ncas supplies an internal preamp cascode voltage, and Vrset controls the placement of the DC reset point of the preamp. Under normal conditions, no connection to these pads is necessary.

Several other diagnostic pads are available for chip testing, including (P127), (R127), and (W127). These are buffered versions of the Ch. 127 preamp output, pipeline read amp output, and pipeline write amp output (see Figure 1 for locations). The buffers are simple PMOS followers that require external bias (a pull-up resistor of $1000 \mathrm{ohms}$ with a $6 \mathrm{~V}$ voltage has proven adequate). Without an external pull-up, the buffer will be inactive. 


\subsubsection{The Back-End}

The SVX4 back end was designed at LBL and Padova, Italy and mates with the SVX4 front end, designed at Fermilab, to produce a complete SVX4 128 channel silicon detector readout chip. The back end contains 128 identical channels of comparators followed by an analog delay and latch, a common ramp generator for all 128 channels, a specially designed comparator common to all 128 channels used for common mode noise discrimination (also called dynamical pedestal subtraction (DPS), and shown in Figure 8), and a FIFO with control logic for nearest neighbor logic, digital thresholds and sparsification. After a trigger signal or a Level 1 Accept (L1A) is received, every channel is digitized simultaneously, passed through the FIFO control logic, and then read out.

Depending of the mode of the chip, two different actions occur. For the $\mathrm{D} \varnothing$ mode, the front end signal acquisition stops while the back end digitizes and readout is completed. For the CDF mode, front end signal acquisition continues simultaneously while digitization and readout is occurring. This "dead timeless operation" therefore requires the use of two independent clocks (FEClk and $\mathrm{BEClk})$ while for $\mathrm{D} \varnothing$ mode only one clock is needed $(\mathrm{FEClk}=\mathrm{BEClk}=\mathrm{Clk})$ that changes frequency accordingly. Operation of the back end requires a $2.5 \mathrm{~V}$ supply, a back end clock, and a few digital control lines.

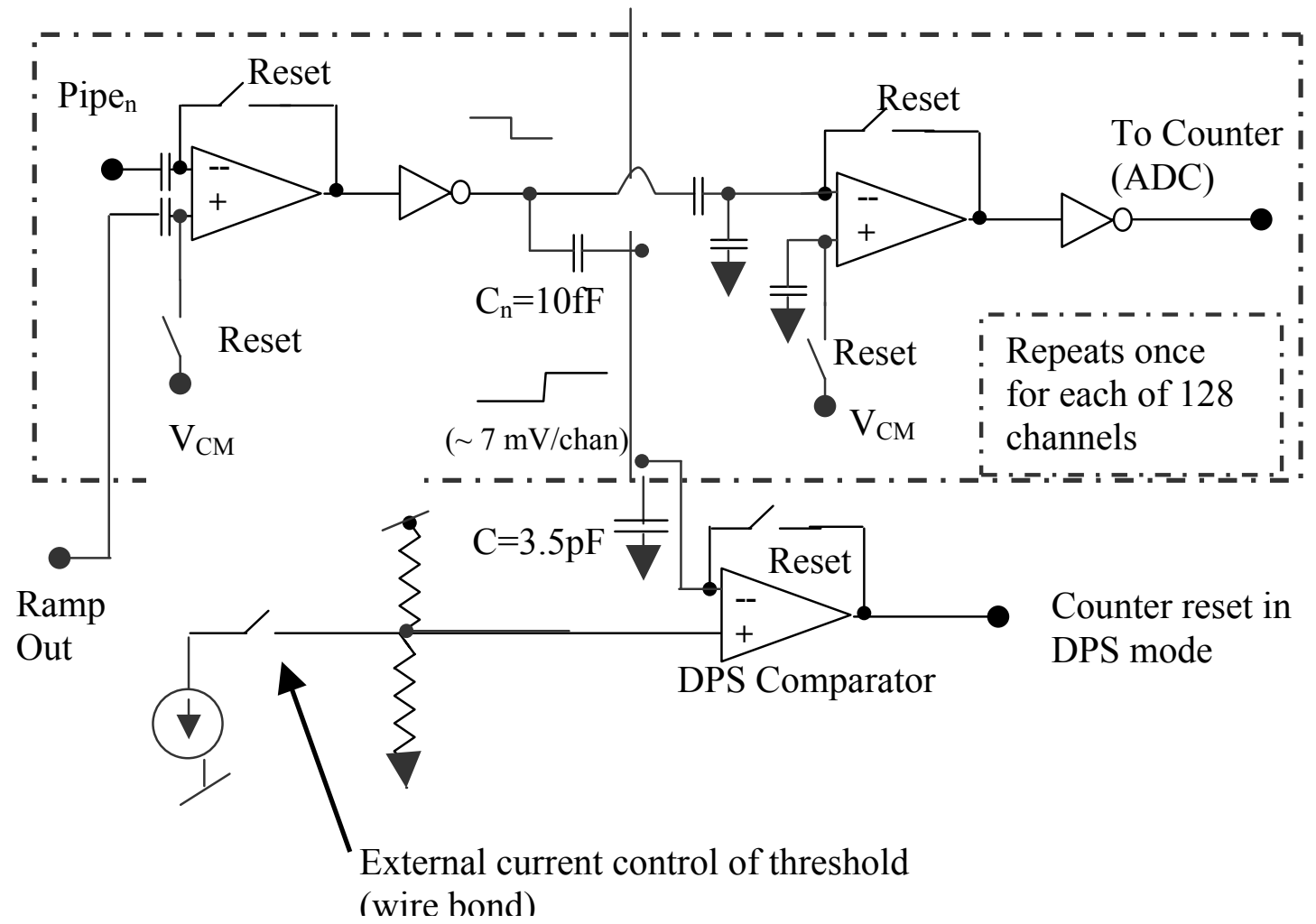

Figure 8 Dynamic (Real Time) Pedestal Subtraction / ADC Block diagram - For each channel the selected pipeline cell is compared with the generated ramp which is common to all channels. The output of the first comparator feeds a second comparator that serves only as a delay circuit. The output of the second comparator is used to latch the Gray counter value and thus provides a measure of the charge on the pipeline capacitor. In addition, the output of the first comparator for all channels is added at the summing junction of the common DPS comparator. When enough channel comparators have fired, the DPS comparator fires in turn and releases the Gray counter which until then is continuously reset when the DPS feature is enabled. The number of channels required to fire the DPS comparator can either be determined by an externally supplied bias or via an internal resistor network. 
For completeness, while the chip is in the Initialize cycle, the front end clock signal is routed to clock a 46 bit shift register, which is downloaded with program bits from the PRin line. Seven of those bits set the chip ID, 3 bits are used for Vcal switching, 1 bit controls the DPS mode, 4 bits set the digital function of the FIFO, 25 bits set the operation parameters of the ADC, and 5 bits set the driver currents for the SVX4 for OBDV and readout data lines. These bits are transferred to an SEU (Single Event Upset due to the passage of an ionizing particle) tolerant shadow register.

The back end has two cycles of operation: Digitization and Readout. During the Digitize cycle, the back end clock (BEClk) is routed to a counter (nominally during Digitize $\mathrm{f}=53 \mathrm{MHz}$, but since both edges of the clock are used the effective digitization rate is $106 \mathrm{MHz}$ ). Proper operation of the ADC is preceded by the manipulation of the ADC control lines.

( Note: for D0 mode, the control lines are multiplexed over the bus lines while for CDF mode these control lines are independent of the bus lines.) We show the proper timing of the control lines in Figure 9.

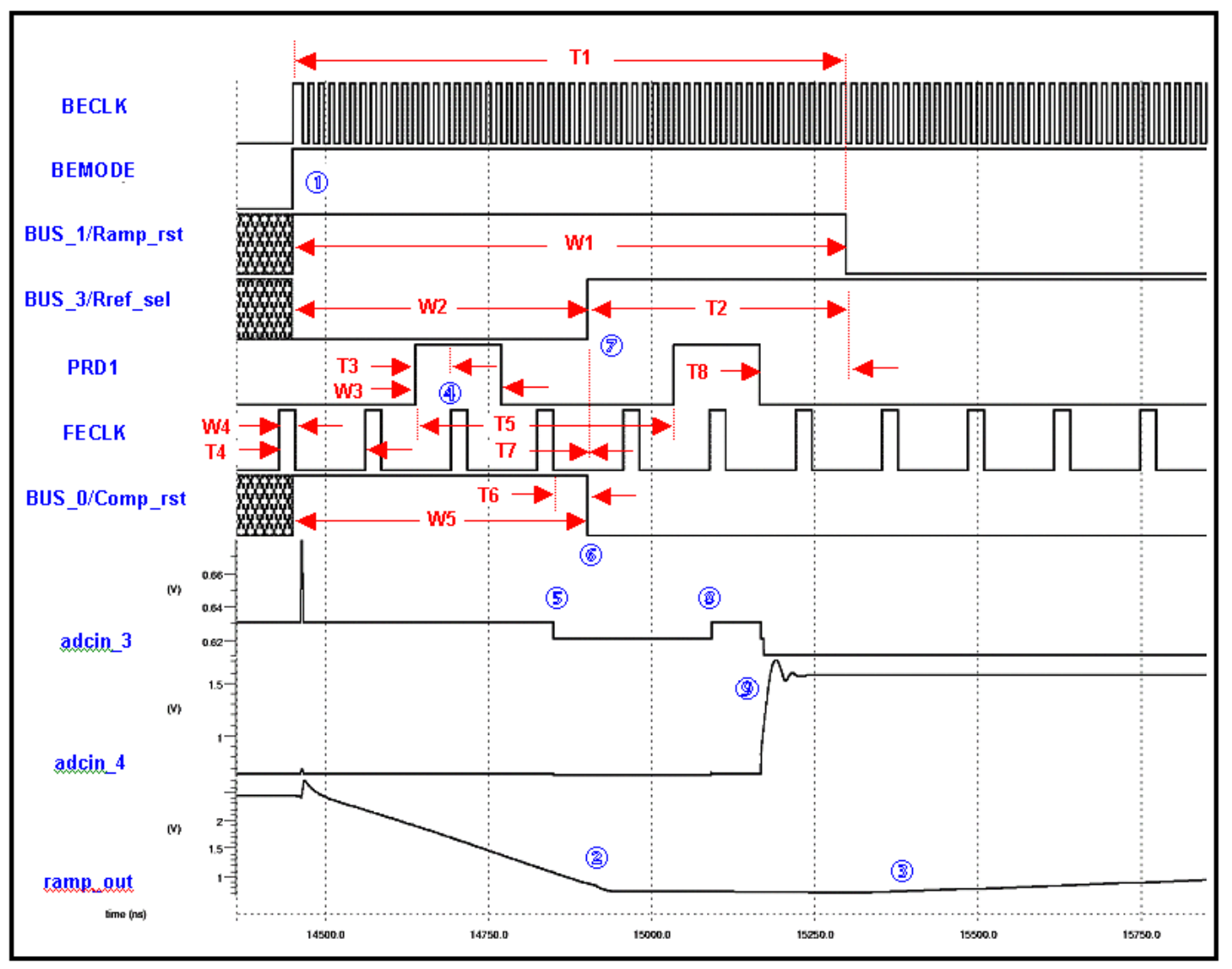

Figure 9 Timing diagram for pipeline read and ADC operations.

The pipeline read/ADC timing consists of two sets of interdependent signal sequences. One is the ADC Ramp Setup (BECLK/Ramp_rst/Rref_sel), and the other Pipeline Read (PRD1/FECLK/Comp_rst). The ADC Ramp Setup controls the ADC ramp generator, the selection of the ADC ramp reference voltage (fixed) or the ADC ramp pedestal (programmable), and start of the ADC counter when not in Real-Time Pedestal Subtraction mode (RTPS). The Pipeline Read sequence controls the signal/pedestal CDS of the pipeline read amplifier on one terminal of the ADC input comparator, as 
well as the ADC ramp sampling on the other. Significant features of the sequences are described below and use references to Figure 9 and Figure 10.

\section{ADC Ramp Setup}

1) Pipeline read and signal digitization is initiated by entering DIGITIZE mode, that is, by asserting (FEMODE $=1+\mathrm{BEMODE}=1$ ) under $\mathbf{C H M O D E}=1$ [1]]. If CHMODE $=0$, DIGITIZE will instead be entered at $\uparrow$ CHMODE when (FEMODE $=1+$ BEMODE $=1)$. This behavior is the result of the MODE pins being processed through a transparent D-latch, which is controlled by CHMODE. MODE changes must occur on or about $\uparrow$ BECLK, or while BECLK $=0$. Upon entering DIGITIZE, the I/O pins BUS_0, BUS_1, BUS_3, change function to Comp_rst, Ramp_rst, RRef_sel, respectively.

2) Asserting RRef_sel $=0$ while Ramp_rst $=1$ resets the ADC ramp to the fixed ADC ramp reference voltage level [(2], which is above the programmable ramp pedestal voltage level. RRef_sel=0 while Ramp_rst $=1$ must then be asserted in concert with Comp_rst, as described below, in order to subtract the programmed pedestal value from the ramp reference voltage. Asserting Ramp_rst=1 also asserts the internal counter reset signal Cntr_Rst when RTPS mode is off.

3) When Ramp_rst is de-asserted the ADC ramp commences in the programmed direction [3]. If RTPS mode is off, the internal signal Cntr_Rst will also be de-asserted. If RTPS mode is selected, Cntr_Rst is controlled by the dynamic threshold comparator circuit. In this case, the counter will be held in reset until the dynamic threshold comparator fires, sometime after the ADC ramp is initiated.

\section{Pipeline Read}

4) The pipeline signal/pedestal sampling sequence is initiated by $\uparrow F E C L K$ under PRD1=1. The relationship of PRD1 and FECLK is fixed in terms of the state of PRD1 during the phases of FECLK, as specified above. The $1^{\text {st }} \uparrow$ FECLK under PRD1 begins the cycle [4]. In the above example, the programmed order ("PB" config bit) is pedestal/signal.

5) During time adc.T6, the pipeline pedestal values are sampled onto the ADC input capacitors while the ADC comparator inputs (the other terminal of the input capacitors) are reset to a fixed internal reference level. Concurrently, during time adc.W5 the ramp reference level is being sampled onto identical comparator input capacitors on the other comparator input terminal. It can be seen on the internal signal adcin_3 (no-hit channel) that the pipeline pedestal value is available to the ADC at the $2^{\text {nd }} \downarrow$ FECLK [5] $]$.

6) When Comp_rst is de-asserted the ADC comparators are un-reset [6]. The ADC input sampling capacitors are now pre-charged to the pipeline pedestal values. From this time on, the ADC input is reading the difference of the pipeline output and the sampled pipeline pedestal, thus the CDS cycle is complete. However, the correct pipeline signal value will not be applied until the $2^{\text {nd }} \downarrow$ PRD1.

7) $\uparrow$ RRef_sel applies the desired offset to the other ADC comparator input capacitor []. This action must take place after $\downarrow$ Comp_rst (adc.T7) in order to achieve the desired CDS operation (rampref-rampped) on the ramp. Note that the effect of this CDS operation is to pre-charge a small offset across the ramp capacitor, which the ramp must "make-up" before it achieves zerocrossing of the original ramp reference level value. The purpose of this process is to allow the ramp to slew for a small period into its linear region, so that the ADC comparators will fire within the linear region of the ramp for small input signals. This is especially important for accurate noise measurement.

8) On the $3^{\text {rd }} \uparrow$ FECLK, under the $2^{\text {nd }}$ PRD1=1, the pipeline pedestal value is de-asserted by the pipeline read amp [8]. On the $2^{\text {nd }} \downarrow$ PRD1 the signal values are asserted by the pipeline read 
amp [(9) $\{$ I am not sure if this is correct-the simulation shows this is the case, but Tom's measurements on the pipeline test chip indicate that it comes on falling FECLK just like the pedestal values_Brad\}. This can be clearly seen on the internal signal adcin_4 (hit channel) above. Time adc.T8 is required to allow the pipeline read amp to settle (and hence the signalpedestal value at the ADC input) before starting the conversion.

\begin{tabular}{|c|c|c|c|c|}
\hline $\begin{array}{l}\text { Timing } \\
\text { Spec }\end{array}$ & Description & Min & Nom & Max \\
\hline adc.T1 & $1^{\text {st }} \uparrow$ BECLK to $\downarrow$ Ramp_rst & 4* BECLK & $900 \mathrm{nS}$ & -- \\
\hline adc.W1 & Width of Ramp_rst in DIGITIZE mode & $600 \mathrm{nS}$ & $900 \mathrm{nS}$ & -- \\
\hline adc.W2 & Width of RRef_sel low under Ramp_rst & $500 \mathrm{nS}$ & $600 \mathrm{nS}$ & -- \\
\hline adc.T2 & $\uparrow$ RRef_sel to $\downarrow$ Ramp_rst & $200 \mathrm{nS}$ & $300 \mathrm{nS}$ & -- \\
\hline adc.T3 & $\uparrow P R D 1$ to $1^{\text {st }} \uparrow F E C L K$ of pipeline read & $5 \mathrm{nS}$ & $54 \mathrm{nS}$ & -- \\
\hline adc.W3 & Width of a PRD1 & $30 \mathrm{nS}$ & $1 *$ FECLK & -- \\
\hline adc.W4 & Width of FECLK high & $20 \mathrm{nS}$ & $25 \mathrm{nS}$ & -- \\
\hline adc.T4 & Period of FECLK & $65 \mathrm{nS}$ & $132 \mathrm{nS}$ & -- \\
\hline adc.T5 & Time between two $\uparrow$ PRD1 for pipeline read & 3* FECLK & 4* FECLK & -- \\
\hline adc.T6 & $2^{\text {nd }} \downarrow$ FECLK of pipeline read to $\downarrow$ Comp_rst & $55 \mathrm{nS}$ & $132 \mathrm{nS}$ & -- \\
\hline adc.T7 & $\downarrow$ Comp_rst to $\uparrow R R e f \_s e l$ & $50 \mathrm{nS}$ & $50 \mathrm{nS}$ & -- \\
\hline adc.W5 & Width of Comp_rst & $100 \mathrm{nS}$ & $455 \mathrm{nS}$ & -- \\
\hline adc.T8 & $2^{\text {nd }} \downarrow$ PRD1 to $\downarrow$ Ramp_rst & $100 \mathrm{nS}$ & $132 \mathrm{nS}$ & -- \\
\hline
\end{tabular}

Table 4 Timing for the various signals for pipeline readout and ADC setup. Items marked by ** are critical for proper operation of the SVX4.

9) The slope of the ramp is determined by the value of the external resistor connected to the ISLOPE pad and the values of the RampRng control variable. For the nominal external resistor of $36 \mathrm{kOhm}$ and with RampRng $=0$ this is $0.5 \mathrm{mV} / \mathrm{nsec}$. This sets the sensitivity of the ADC, i.e. number of ADC counts per electron. Though the operating frequency of the back end clock during digitization is set to $106 \mathrm{MHz}$, this frequency can be altered and therefore the number of ADC counts per electron is altered as well. Approximately, the conversion is for read all mode is given by:

$$
\begin{aligned}
\mathrm{ADC} \text { counts }= & \text { ADC delay } / 2 * \mathrm{f}(\mathrm{BEClk})+\text { pedestal } / 2 * \mathrm{f}(\mathrm{BEClk}) \\
& +(\mathrm{Q} * 15 \mathrm{mV} / \mathrm{fC}) / \text { Ramp Rate }(\text { Rext }) / 2 * \mathrm{f}(\text { BEClk })
\end{aligned}
$$

and the conversion for DPS mode is given by:

$$
\mathrm{ADC} \text { counts }=\mathrm{ADC} \text { delay } / 2 * \mathrm{f}(\mathrm{BEClk})+(\text { Num of e's } / \mathrm{C}) / \text { Ramp Rate }(\mathrm{R} \mathrm{ext}) / 2 * \mathrm{f}(\mathrm{BEClk}) .
$$




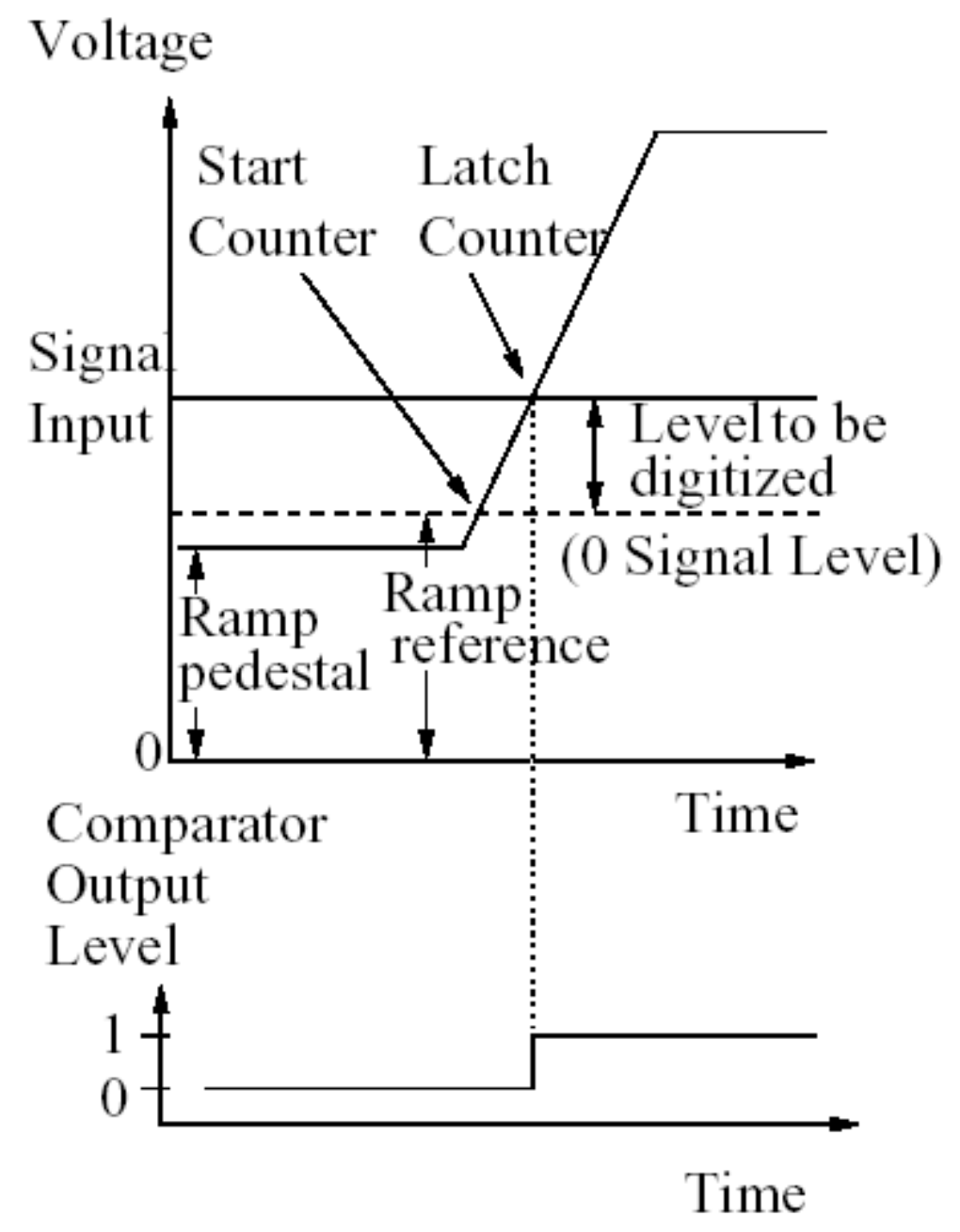

Figure 10 Comparator operation for the default polarity (000). Ramp reference is set either externally or via an internal voltage source.

If sparsification is on, the digital threshold that is downloaded to the chip is used as a semaphore to tag the channels for readout.

In Readout Cycle, the FIFO drops a token that is systematically passed to each individual channel sequentially. A channel is flagged for readout if the value stored during Digitize is over the threshold value, or Read Neighbor is set and the neighbor value is over the digital threshold, or Read All is set or Read Channel 63 or Read Channel 127 are set. Timing considerations as outlined in section 1.3 force us to read channel 63 at all times

\section{The Difference Between D0 and CDF Modes}

The SVX4 has the ability to operate in two modes: D0 mode and CDF mode. What does that mean? 
Physically, there is an external pad on the chip that must be wirebonded to AVDD/DVDD or GND. If this pad is not bonded correctly, there is no guarantee the chip will function properly (even though the level is pulled weakly to GND - i.e. to CDF mode). If the chip is bonded to AVDD/DVDD the chip will operate in D0 mode. When the chip is in D0 mode, it does not have the ability to acquire data while digitizing or readout. Internal to the chip, this means that the front end clock is not gated when the chip is in readout mode. It is necessary only to drive the front end clock during initialize and acquire. During digitize only the back end clock is needed and similarly for readout. All 46 pipeline cells are available, but there is no secondary pipeline to store events. D0 necessarily wirebonds the front end and back end clocks together meaning there is only one clock in the system.

If this pad is grounded, then the chip is in CDF mode. This means that the chip can acquire data and digitize simultaneously. In order to have this functionality requires the use of two independent clocks. Physically, this means the back end clock maybe operating while the front end clock is running at a different frequency. The pattern for setting up the ADC is similar, but the control signals are transmitted differently.

In D0 mode, the control lines for PRD1, PRD2, L1A, CALSR, RMPRST, RREFSEL, and COMPRST are multiplexed over the differential bus lines. In CDF mode, the control signals PRD1, PRD2, L1A, and CALSR are transmitted through independent control lines, while the RMPRST, RREFSEL, and COMPRST are multiplexed over the bus lines identical to D0 mode. By correctly wirebonding the D0 mode pad, a latch inside the chip is set that determines where the control signals will be taken from.

It should be noted that the chip has been operated successfully in a hybrid mode - i.e. in a two buffer mode. In such a case the D0/CDF wire bond pad was controlled externally, i.e. the chip could be put in either mode on the fly. Both front end and back end clocks were provided. The chip was set to CDF mode and data was acquired in the usual fashion. by the front end clock. When a trigger occurred, the external sequencer switched to D0 mode, issued L1 accept, and two PRD1 signals to set up the chip to readout the first capacitor and then switched back to CDF mode. Digitize and readout worked as they did during normal operation. If another L1 trigger occurred during digitize and read out, the front end clock halted to preserve the L1 data and raise L1busy to stop additional triggers. After completing readout, the sequencer switched back to D0 mode and issued a PRD2. If there was a pending trigger, it would follow with 2 PRD1's to set up to digitize the signal. 


\section{Initialization Bit Stream}

In Table 5 and Table 6, we list the bit assignment for the SVX4 chip.

SVX4 Configuration Register Table

\begin{tabular}{|c|c|c|c|c|}
\hline $\begin{array}{c}\text { Bit } \\
\text { Number }\end{array}$ & Bit Name & Description & Values & $\begin{array}{l}\text { Nominal } \\
\text { Setting }\end{array}$ \\
\hline \multicolumn{5}{|c|}{ — Front end Bit Assignments - } \\
\hline $0: 127$ & $\begin{array}{l}\text { Mask } \\
{[127: 0]}\end{array}$ & $\begin{array}{c}\text { Cal mask or channel disable } \\
\text { register (see bit } 130 \text { for } \\
\text { assignment) }\end{array}$ & $\begin{array}{c}0=\text { mask } / \text { enable } \\
1=\text { unmask } / \text { disable }\end{array}$ & $0 \ldots 0$ \\
\hline 128 & spare & spare & $\mathrm{X}$ & $\mathrm{X}$ \\
\hline 129 & VCAL & $\begin{array}{l}\text { Connects the VCAL pad to the } \\
\text { internal voltage divider }\end{array}$ & $\begin{array}{c}0=\text { not connected to pad } \\
1=\text { connected to pad }\end{array}$ & 1 \\
\hline 130 & Disable & $\begin{array}{c}\text { Select whether mask reg acts as a } \\
\text { channel disable reg or a cal mask } \\
\text { reg }\end{array}$ & $\begin{array}{c}\quad 0=\text { cal mask } \\
1=\text { channel disable }\end{array}$ & 0 \\
\hline $131: 134$ & $\begin{array}{l}\mathrm{BW} \\
{[0: 3]}\end{array}$ & $\begin{array}{l}\text { Preamp rise time adjustment } \\
\text { (depends on input capacitance), } \\
\text { binary weighted }\end{array}$ & $\begin{array}{c}\text { For } \operatorname{Cin}=10 \mathrm{pF}: \\
\mathrm{Tr} \approx 25 \mathrm{nS}+(\mathrm{BW} * 4 \mathrm{nS}) \\
\text { For } \operatorname{Cin}=50 \mathrm{pF}: \\
\mathrm{Tr} \approx 60 \mathrm{nS}+(\mathrm{BW} * 10 \mathrm{nS})\end{array}$ & 0010 \\
\hline $135: 138$ & $\begin{array}{c}\text { Isel } \\
{[0: 3]}\end{array}$ & $\begin{array}{l}\text { Preamp input FET bias current } \\
\text { adjustment, binary weighted }\end{array}$ & $\begin{array}{c}\text { Bias current } \approx 164 \mathrm{uA}+(\text { Isel } * \\
32 \mathrm{uA})\end{array}$ & 0010 \\
\hline $139: 140$ & IWsel $[0: 1]$ & $\begin{array}{l}\text { Pipeline write amp bias current } \\
\text { adjustment, NOT binary weighted }\end{array}$ & $\begin{array}{c}\text { Bias current } \approx 26 \mathrm{uA}+(\mathrm{IWsel0} * \\
26 \mathrm{uA})+ \\
(\mathrm{IWsel} 1 * 26 \mathrm{uA})\end{array}$ & 10 \\
\hline $141: 142$ & IRsel $[0: 1]$ & $\begin{array}{c}\text { Pipeline read amp bias current } \\
\text { adjustment, binary weighted }\end{array}$ & $\begin{array}{l}\text { Bias current } \approx 26 \mathrm{uA} \\
+(\text { IRsel } * 13 \mathrm{uA})\end{array}$ & 10 \\
\hline $143: 148$ & $\begin{array}{l}\text { PickDel } \\
{[0: 5]}\end{array}$ & $\begin{array}{c}\text { Trigger latency; select system L1A } \\
\text { delay as a number of FEClk } \\
\text { periods }\end{array}$ & $0 \ldots 42$ & TBD \\
\hline 149 & $\mathrm{~PB}$ & Pipeline readout order & $\begin{array}{r}0=\text { pedestal }, \text { signal } \\
1=\text { signal, pedestal }\end{array}$ & 0 \\
\hline \multicolumn{5}{|c|}{ — Back end Bit Assignments - } \\
\hline $150: 156$ & $\begin{array}{c}\text { ID } \\
{[6: 0]}\end{array}$ & Chip ID assignment & $0 \ldots 127$ & TBD \\
\hline 157 & RTPS & $\begin{array}{c}\text { Real Time Pedestal Subtraction } \\
\text { disable }\end{array}$ & $\begin{array}{l}0=\text { RTPS on } \\
1=\text { RTPS off }\end{array}$ & 0 \\
\hline 158 & $\operatorname{Rd} 127$ & $\begin{array}{c}\text { Always readout channel } 127 \\
\text { regardless of hit status }\end{array}$ & $\begin{array}{l}0=\mathrm{Rd} 127 \text { off } \\
1=\mathrm{Rd} 127 \text { on }\end{array}$ & 0 \\
\hline 159 & Rd63 & $\begin{array}{l}\text { Always readout channel } 63 \\
\text { regardless of hit status }\end{array}$ & $\begin{array}{l}0=\operatorname{Rd} 63 \text { off } \\
1=\operatorname{Rd} 63 \text { on }\end{array}$ & 0 \\
\hline 160 & RdAll & Always readout all channels & $\begin{array}{l}0=\text { RdAll off } \\
1=\text { RdAll on }\end{array}$ & 0 \\
\hline 161 & RdNeigh & $\begin{array}{l}\text { Readout hit channels and their } \\
\text { neighbors }\end{array}$ & $\begin{array}{l}0=\text { RdNeigh off } \\
1=\text { RdNeigh on }\end{array}$ & 1 \\
\hline
\end{tabular}

Table 5 Bit assignment for the SVX4 chip. 


\begin{tabular}{|c|c|c|c|c|}
\hline $\begin{array}{c}\text { Bit } \\
\text { Number }\end{array}$ & Bit Name & Description & Values & $\begin{array}{l}\text { Nominal } \\
\text { Setting }\end{array}$ \\
\hline $162: 165$ & $\begin{array}{c}\text { RampPed } \\
{[0: 3]}\end{array}$ & $\begin{array}{c}\text { ADC ramp pedestal setting, binary } \\
\text { weighted }\end{array}$ & $\begin{array}{c}\text { RampDir }=0: \text { Ped } \approx 480 \mathrm{mV} \\
\text { +(RampPed * } 23 \mathrm{mV}) \\
\text { RampDir }=1: \text { Ped } \approx 1.8 \mathrm{~V} \\
\text {-(RampPed } * 23 \mathrm{mV})\end{array}$ & 0001 \\
\hline 166 & RampDir & $\begin{array}{l}\text { ADC ramp direction, ramp up or } \\
\text { ramp down }\end{array}$ & $\begin{array}{c}0=\text { ramp up } \\
1=\text { ramp down }\end{array}$ & 0 \\
\hline 167 & CompPol & $\begin{array}{l}\text { Comparator polarity; sets } \\
\text { comparator and delay input for } \\
0 \rightarrow 1 \text { or } 1 \rightarrow 0 \text { transition }\end{array}$ & $\begin{array}{l}0=0 \rightarrow 1(\text { for RampDir }=0) \\
1=1 \rightarrow 0(\text { for RampDir }=1)\end{array}$ & 0 \\
\hline $168: 170$ & $\begin{array}{c}\text { RampRng } \\
{[0: 2]}\end{array}$ & $\begin{array}{l}\text { ADC ramp range, adjusts slope of } \\
\text { ramp }\end{array}$ & $\begin{array}{c}\text { Slope } \approx 0.5 \mathrm{mV} / \mathrm{nS} * \\
{[1+(2 * \mathrm{r} 0)+(2 * \mathrm{r} 1)+(1 * \mathrm{r} 2)]^{-1}}\end{array}$ & 000 \\
\hline $171: 178$ & $\begin{array}{c}\text { Thresh } \\
{[7: 0]}\end{array}$ & $\begin{array}{l}\text { ADC digital threshold setting, } \\
\text { Gray code }\end{array}$ & $0 . .255$ & TBD \\
\hline $179: 186$ & $\begin{array}{l}\text { CntrMod } \\
\quad[7: 0]\end{array}$ & $\begin{array}{l}\text { Counter Modulo, sets counter } \\
\text { value at which overflow occurs, } \\
\text { Gray code }\end{array}$ & $0 . .255$ & TBD \\
\hline 187 & $\mathrm{FC}$ & $\begin{array}{l}\text { First Chip flag; enables the first } \\
\text { chip to drive OBDV before } \\
\text { readout begins }\end{array}$ & $1=$ this is the first chip & 0 \\
\hline 188 & $\mathrm{LC}$ & $\begin{array}{c}\text { Last Chip flag; enables the last } \\
\text { chip to drive OBDV after readout } \\
\text { ends }\end{array}$ & $1=$ this is the last chip & 0 \\
\hline 189:191 & $\begin{array}{c}\text { DriverI } \\
{[2: 0]}\end{array}$ & $\begin{array}{l}\text { Output driver current select; } \\
\text { selects output series resistance; the } \\
\text { resistance selected appears in } \\
\text { series on EACH output pin (plus } \\
\text { and minus) }\end{array}$ & $\begin{array}{c}\mathrm{R} \approx[(\mathrm{d} 2 / 43)+ \\
(\mathrm{d} 1 / 86)+(\mathrm{d} 0 / 172)]^{-1} \\
\text { Drivers off if DriverI }=0\end{array}$ & 111 \\
\hline
\end{tabular}

Table 6 Bit assignment for the SVX4 chip.

Notes: 1)The correspondence of the bus notation indicies are preserved in the table above from column-to-column, i.e. for "Bit Number 162:165," RampPed [3] corresponds to Bit 165, which corresponds to a "1" in the "Nominal Setting" column. This correspondence explicitly determines whether the LSB or MSB of a bus loads first, since there is no common rule.

2)The "Bit Number" references under "Frontend Configuration Register Bit Assignments" are reversed with respect to the "SVX4 Front End" document, in order to accommodate a contiguous, ascending bit order for the complete configuration register.

3)Bit 0 loads first.

4) $\mathrm{Vped}=$ Vref - (11 - setting) $* 23 \mathrm{mV}$. This actually holds for settings from 0 to 14 . When going from 14 to 15 , the step is 6 times the nominal step, or $138 \mathrm{mV}$.For a positive going ramp, we want Vref to be lower than Vped so that the ramp must go for a while before flipping the comparator, thus we normally use settings less than 11. Settings greater than 11 would be used for opposite polarity operation. 11 is the "zero" setting, and theoretically the measured pedestal for this setting would just give the (comparator delay + analog delay), which nominally in simulation for versB is $120 \mathrm{~ns}$. At $10 \mathrm{~ns} /$ count this would give 12 counts.

\subsection{SVX4 Configuration Register Explanation}

Below, we give an extended discussion of the bits that are downloaded into the configuration register. 
0 : $\mathrm{PB}$ (pipeline readout polarity bit). $0=$ pedestal - signal, $1=$ signal - pedestal .

1-6: Pipeline level 1 trigger delay. Bit 1 is MSB, bit 6 is LSB. Valid range is 1-42.

7-8: IRSel1-0 (pipeline read amp bias current select). Read amp bias current $=13 \mathrm{uA}+$ $($ IRSel0)*(13 uA $)+($ IRSel1 $) *(26 \mathrm{uA})$. Increasing the read amp bias current simply speeds up the risetime. The lowest current is probably acceptable.

9-10: IWSel1-0 (pipeline write amp bias current select). Write amp bias current $=26 \mathrm{uA}+$ $($ IRSel0)*(26 uA $)+($ IRSel1)*(26 uA). Increasing the write amp bias current speeds up the pipeline reset speed and the pipeline risetime. Nominal bias current $=52 \mathrm{uA}$.

11-14: Isel3-0 (preamp input transistor bias current select). Bias current $=164 \mathrm{uA}+$ $(\mathrm{Isel} 3) *(256 \mathrm{uA})+($ Isel2 $) *(128 \mathrm{uA})+($ Isel1 $) *(64 \mathrm{uA})+($ Isel0 $) *(32 \mathrm{uA})$.

15-18: BW3-0 (preamp bandwidth). Used to adjust preamp risetime. Risetime will depend on input capacitance, bias current, and bandwidth setting. The bits are binary weighted: BW0 $=\mathrm{LSB}, \mathrm{BW} 3=\mathrm{MSB}$.

19: Mask/Disable. If Mask/Disable = low, then the 128 bit channel register functions as a mask register for test charge injection (register bit $=$ high to enable charge injection). If Mask/Disable $=$ high, then the 128 bit channel register functions as a channel disable register (register bit $=$ high to disable channel).

20-147: Channel register $<0: 127>$

\subsection{The Output Data Format for the SVX4}

The SVX4 generates an 8-bit (byte) output on the 8 differential data bus lines (numbered as BUS0 to BUS7 or DATA0 to DATA7 and their differential complements, 0 being least significant bit) on every transition of the OBDV, the positive side of the differential Output Data Valid signal level. Thus data is presented on both positive-to-negative and negative-to-positive transitions of OBDV. The edges of the transitions of the BUS lines and OBDV are simultaneous at the output of the SVX4 chip; it is left for the readout electronics to generate the appropriate delay on OBDV and properly strobe the BUS lines. The Chip ID byte appears on the first negative-to-positive OBDV transition. The Pipeline Id appears on the first positive-to-negative transition of OBDV.

The output stream is of variable depth depending on many parameters (e.g. threshold, read all, read neighbor, read 64, etc.) and on the amount of true data (or noise!) seen at the input of the SVX4. As long as valid data is being generated by a chip the PRIOUT is asserted high, and a chip will assert PRIOUT and generate data on the BUS lines and toggle OBDV only if the PRIIN is low; PRIIN is weakly pulled to low via an internal resistor. These assignments allow for the daisy-chained readout described in more detail in Section 5. Finally, it should be noted that OBDV is nothing more but an appropriately regenerated and delayed form of the BECLOCK, which is used to clock the data on the output BUS lines. The SVX4 data output is shown in Table 7. 


\section{Output Stream Format:}

\begin{tabular}{|l|l|l|}
\hline Byte no & Content & Comments \\
\hline 1 & Chip ID & $\begin{array}{l}\text { Has highest bit set to 1, the rest are derived } \\
\text { from the downloaded parameters } \\
\text { (i.e. lowest value in Hex is "80") }\end{array}$ \\
\hline 2 & Pipeline Cell Number & $\begin{array}{l}\text { True (physical) number of cell being digitized } \\
\text { Can be } 1 \text { to } 42 \text { decimal (but not } 0), \text { the two } \\
\text { most significant bits are always } 0 .\end{array}$ \\
\hline 3 & Channel Id & Can be 0 to 7F hex (127 decimal), increasing. \\
\hline 4 & Data for above Channel Id & Gray Coded (0 to 255 decimal) \\
\hline$\ldots$ & $\ldots$ & \\
\hline Last-1 & Channel Id & Can be 0 to FE hex (127 decimal), increasing. \\
\hline Last & Data for Above Channel Id & Gray Coded (0 to 255 decimal) \\
\hline
\end{tabular}

Table 7 Table showing the data output from the SVX4 chip.

Thus the minimal readout is 2 bytes (Chip ID and Pipeline Cell) and no data, presumably due to no channel being above pedestal, and also due to Read All, Read 127, and Read 63 bits being off as well.

\subsubsection{Gray Code information}

In Table 8, we show the algorithm for converting from binary to Gray representations of data and vice versa.

\section{Algorithm for Binary to Gray conversion}

$$
\begin{aligned}
& \text { binary }=\mathbf{B}_{\mathrm{j}} \mathbf{B}_{\mathrm{j}-1} \quad \ldots \quad \mathbf{B}_{1} \mathbf{B}_{0} \\
& \mathbf{B}_{\mathrm{i}} \text { are the binary digits, } \mathrm{i}=0 \text { to } \mathrm{j} \\
& \text { gray }=\mathbf{G}_{\mathrm{j}} \mathbf{G}_{\mathrm{j}-1} \quad \ldots \quad \mathbf{G}_{1} \mathbf{G}_{0} \\
& \mathbf{G}_{\mathrm{j}}=\mathbf{B}_{\mathrm{j}} \text {, and } \mathbf{G}_{\mathrm{i}}=\operatorname{XOR}\left(\mathbf{B}_{\mathrm{i}-1}, \mathbf{B}_{\mathrm{i}}\right)
\end{aligned}
$$

\section{Algorithm for Gray to Binary conversion}

defined in a recursive fashion only

$$
B_{j}=G_{j} \text {, and } B_{i}=\operatorname{XOR}\left(G_{i}, B_{i+1}\right)
$$

Note: $\mathrm{j}$ here is the most significant bit and one works towards the least significant bit

Table 8 Algorithm for converting from the binary representation to the Gray code representation of data and vice versa. 
The embedded MS Excel Spreadsheet in Table 9 allows for an easy translation between the codes, alternatively one can go to the Appendix for the same information.

\section{RED numbers are input}

\section{Use the next four lines to convert between decimal, hex, and binary}

\begin{tabular}{|c|c|c|}
\hline Decimal & Hex & Binary \\
\hline 111 & $6 \mathrm{~F}$ & 1101111 \\
\hline 110 & $6 \mathrm{E}$ & 1101110 \\
\hline 109 & $6 \mathrm{D}$ & 1101101 \\
\hline
\end{tabular}

Now insert the value aobtained above into the appropriate "NORMAL" cells in the lines below

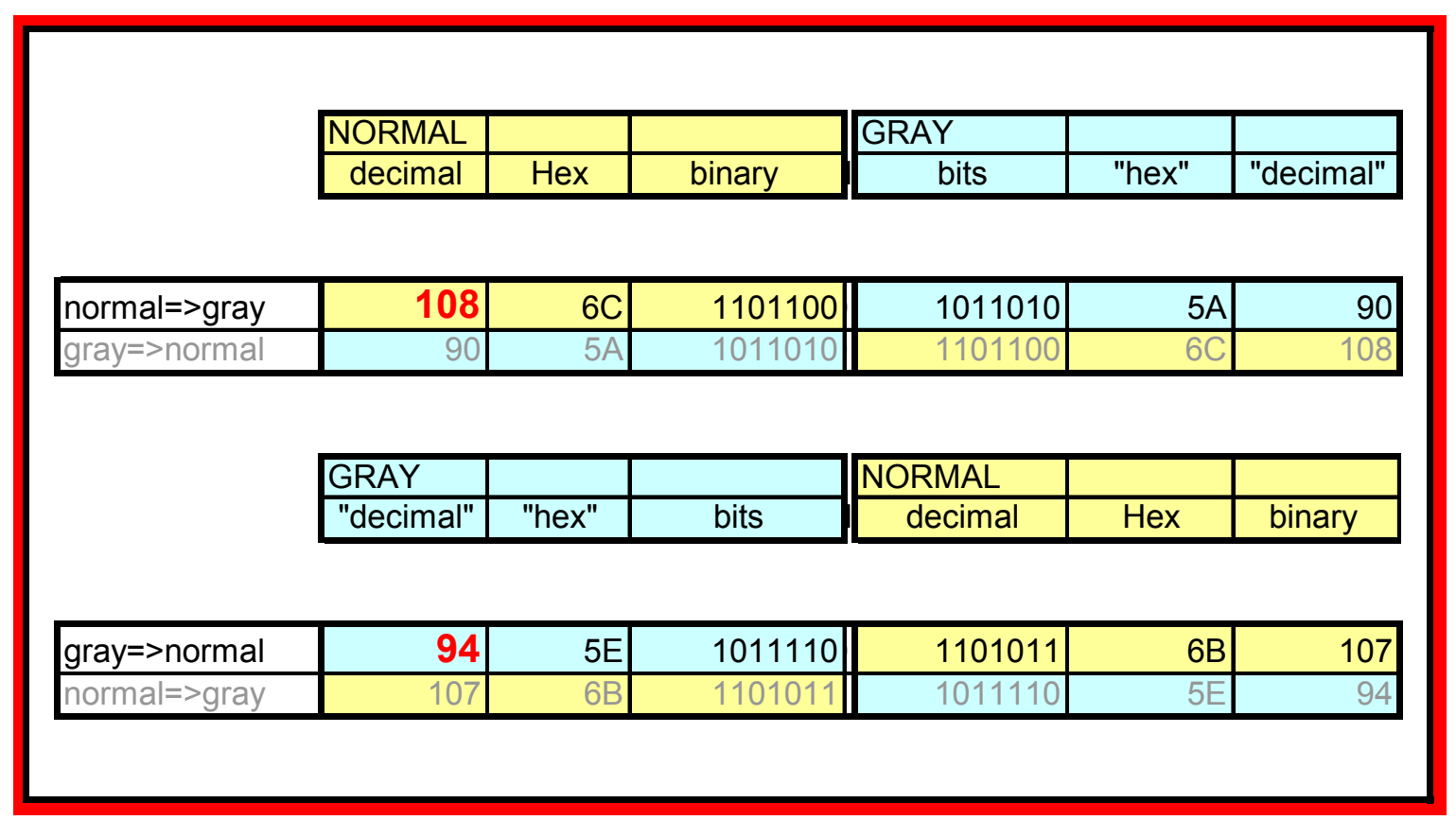

Table 9 Embedded spreadsheet for converting between the binary representation and the Gray code representation. 


\section{Mechanical and Electrical Specifications}

We measured all the mechanical and electrical specifications of the SVX4 chip and they are listed below.

\subsection{Pad layout}

In Figure 11, we show the pad layout for the SVX4 die.

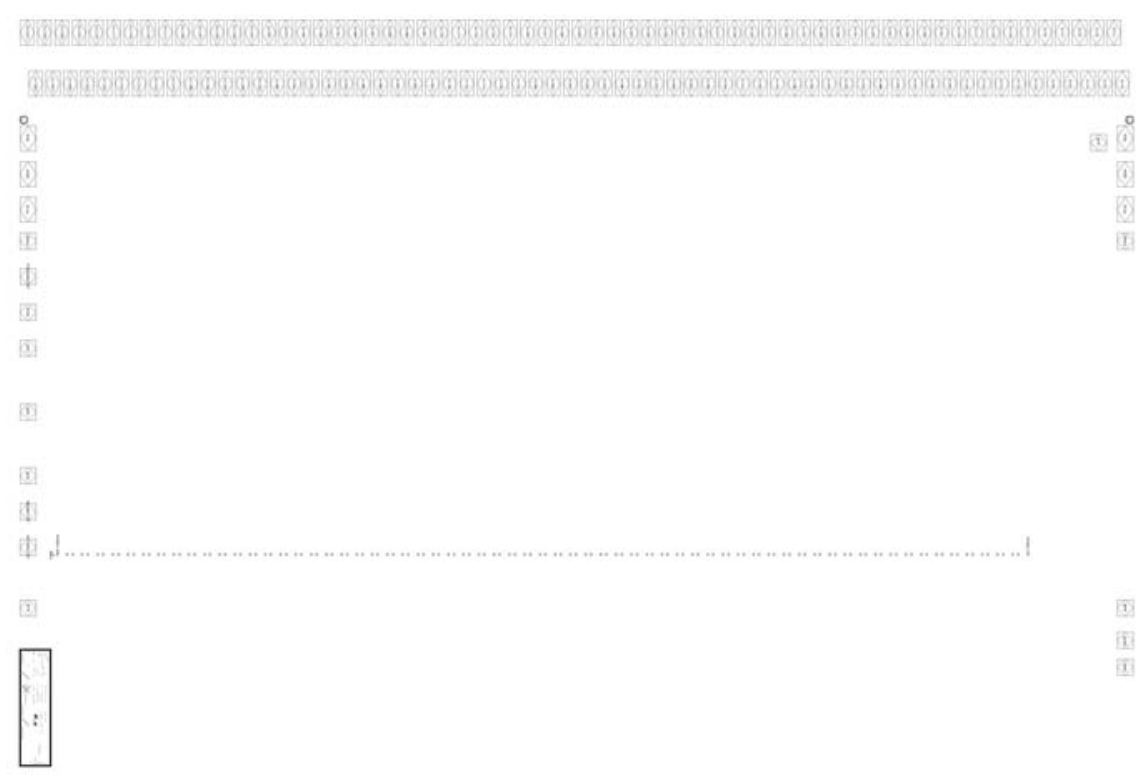

Figure 11 Pad layout on the SVX4

Click here to get a PDF file that you can expand easily 


\subsection{Pin List for the SVX4}

In Table 10 and Table 11, we show the pin list for the SVX4 die.

\begin{tabular}{|c|c|c|c|c|c|c|}
\hline $\begin{array}{l}\text { Pin } \\
\text { Number }\end{array}$ & Pin Name & $\begin{array}{c}\text { Type } \\
\text { Analog } \\
\text { Digital }\end{array}$ & $\begin{array}{c}\text { Type } \\
\text { Input } \\
\text { Output }\end{array}$ & $\begin{array}{l}\text { Nom. } \\
\text { Voltage }\end{array}$ & $\begin{array}{c}\text { Wire } \\
\text { Bonded }\end{array}$ & $\begin{array}{l}\text { Description \& External } \\
\text { Components Required }\end{array}$ \\
\hline 1,79 & VCAL & $\mathrm{A}$ & I & $\begin{array}{l}\text { AVDDfe } \\
\quad \div 4\end{array}$ & Either & Calibration charge setting \\
\hline $\begin{array}{l}2,15,71 \\
78 \\
\end{array}$ & AVDD & A & I & 2.5 & Either & $\begin{array}{l}\text { Analog power supply- } \\
\text { decouple to gnd! } \mathrm{w} / 0.1 \mathrm{uF}\end{array}$ \\
\hline 3,77 & Bias & A & I & 0.8 & Either & $\begin{array}{l}\text { Frontend master bias } \\
\text { reference- decouple to AVDDfe } \\
\text { w/10 nF }\end{array}$ \\
\hline 4,76 & VRset & A & I & 1.0 & No & $\begin{array}{c}\text { Frontend reset level reference } \\
\text { voltage }\end{array}$ \\
\hline 5 & PreampBuf127 & A & $\mathrm{O}$ & -- & No & $\begin{array}{l}\text { Ch127 preamp buffered output; } \\
\text { requires ext resistor }\end{array}$ \\
\hline 6 & Ncas & A & $\mathrm{O}$ & 0.6 & No & \\
\hline $\begin{array}{l}7,8,9,12 \\
13,75 \\
210 \\
\end{array}$ & gnd! & A & I & 0 & No & Analog ground, substrate \\
\hline 10 & ReadBuf127 & A & $\mathrm{O}$ & -- & No & $\begin{array}{l}\text { Ch127 Pipeline Read amplifier } \\
\text { output; requires ext. resistor }\end{array}$ \\
\hline 11 & WriteBuf127 & A & $\mathrm{O}$ & -- & No & $\begin{array}{l}\text { Ch127 Pipeline Write amplifier } \\
\text { output; requires external resistor }\end{array}$ \\
\hline 14,72 & AREF & A & I & 2.5 & Either & $\begin{array}{l}\text { ADC ramp pedestal DAC } \\
\text { reference }\end{array}$ \\
\hline 16,70 & IQUI & A & I & 0.6 & Either & $\begin{array}{c}\mathrm{ADC} \text { and data receiver bias } \\
\text { current setting-7.7k resistor to } \\
\text { AVDDadc }\end{array}$ \\
\hline 17,69 & VTH & A & I & 0.9 & Either & $\begin{array}{l}\text { Dynamic Pedestal Subtraction } \\
\text { threshold voltage setting }\end{array}$ \\
\hline $18,68,74$ & gndd! & $\mathrm{A}$ & I & 0 & $\begin{array}{l}\text { Either } 19 \\
\text { or } 69\end{array}$ & Digital ground \\
\hline $19,67,73$ & vddd! & $\mathrm{A}$ & I & 2.5 & $\begin{array}{l}\text { Either } 20 \\
\text { or } 68\end{array}$ & Digital Vdd \\
\hline 20,66 & D0MODE & $\mathrm{D}$ & I & $0 / 2.5$ & Either & $\begin{array}{l}\text { Connect to vddd! for D0, gndd! } \\
\text { For CDF mode }\end{array}$ \\
\hline 21,65 & USESEU & $\mathrm{D}$ & I & $0 / 2.5$ & Either & $\begin{array}{l}\text { Connect to vddd! to select SEU } \\
\text { register for configuration, or } \\
\text { gndd! for shift register output }\end{array}$ \\
\hline
\end{tabular}

Table 10 Pin listing for the SVX4 chip. 


\begin{tabular}{|c|c|c|c|c|c|c|}
\hline $\begin{array}{c}\text { Pin } \\
\text { Number }\end{array}$ & Pin Name & $\begin{array}{c}\text { Analog } \\
\text { Digital } \\
\text { Diff }\end{array}$ & $\begin{array}{c}\text { Input } \\
\text { Output } \\
\text { I/O }\end{array}$ & $\begin{array}{l}\text { Nom. } \\
\text { Voltage }\end{array}$ & $\begin{array}{c}\text { Wire } \\
\text { Bonded }\end{array}$ & $\begin{array}{l}\text { Description \& External } \\
\text { Components Required }\end{array}$ \\
\hline 22,64 & ISLOPE & A & I & 1.5 & Either & $\begin{array}{l}\text { ADC ramp slope bias-36k } \\
\text { resistor to gnd! }\end{array}$ \\
\hline 23 & BNBR & $\mathrm{D}$ & $\mathrm{I} / \mathrm{O}$ & $0 / 2.5$ & Yes & $\begin{array}{l}\text { Bottom Neighbor; open drain } \\
\text { w/2k internal pull-up }\end{array}$ \\
\hline 24 & PRIOUT & Diff & $\mathrm{O}$ & $0-2.5$ & Yes & Priority Out plus \\
\hline 25 & PRIOUTB & Diff & $\mathrm{O}$ & $0-2.5$ & Yes & Priority Out minus \\
\hline 26,60 & SVDD & $\mathrm{A}$ & $\mathrm{I}$ & 2.5 & Either & Output Driver supply \\
\hline 27,59 & SGND & A & $\mathrm{I}$ & 0 & Either & Output Driver ground \\
\hline 28 & EXTRA & -- & -- & -- & No & Spare pad \\
\hline $\begin{array}{l}29,31,33 \\
35,37,39 \\
\quad 41,43\end{array}$ & $\begin{array}{c}\mathrm{BUSB}<7>, \ldots \\
\mathrm{BUSB}<0>\end{array}$ & Diff & $\mathrm{I} / \mathrm{O}$ & $0-2.5$ & Yes & $\begin{array}{c}\text { Data bus } 7-0 \text { minus (see "Bus } \\
\text { Pin Multiplexing Table" for } \\
\text { secondary pin function by } \\
\text { mode) }\end{array}$ \\
\hline $\begin{array}{l}30,32,34 \\
36,38,40 \\
\quad 42,44\end{array}$ & $\begin{array}{c}\text { BUS }<7>, . . \\
\text { BUS }<0>\end{array}$ & Diff & $\mathrm{I} / \mathrm{O}$ & $0-2.5$ & Yes & $\begin{array}{c}\text { Data bus 7-0 plus (see "Bus } \\
\text { Pin Multiplexing Table" for } \\
\text { secondary pin function by } \\
\text { mode) }\end{array}$ \\
\hline 45 & OBDVB & Diff & $\mathrm{I} / \mathrm{O}$ & $0-2.5$ & Yes & Odd Byte Data Valid minus \\
\hline 46 & OBDV & Diff & $\mathrm{I} / \mathrm{O}$ & $0-2.5$ & Yes & Odd Byte Data Valid plus \\
\hline 47,208 & BECLKB & Diff & $\mathrm{I}$ & $0-2.5$ & Either & Backend Clock minus \\
\hline 48,209 & BECLK & Diff & $\mathrm{I}$ & $0-2.5$ & Either & Backend Clock plus \\
\hline 49 & FECLKB & Diff & $\mathrm{I}$ & $0-2.5$ & Either & Frontend Clock minus \\
\hline 50 & FECLK & Diff & $\mathrm{I}$ & $0-2.5$ & Either & Frontend Clock plus \\
\hline 51 & CHMODE & $\mathrm{D}$ & $\mathrm{I}$ & $0 / 2.5$ & Yes & Change Mode \\
\hline 52 & BEMODE & $\mathrm{D}$ & $\mathrm{I}$ & $0 / 2.5$ & Yes & Backend Mode (Mode 1) \\
\hline 53 & FEMODE & $\mathrm{D}$ & $\mathrm{I}$ & $0 / 2.5$ & Yes & Frontend Mode (Mode 0) \\
\hline 54 & CALSR & $\mathrm{D}$ & I & $0 / 2.5$ & Yes* & $\begin{array}{l}\text { Cal Strobe (Acquire mode) } \\
\text { Write SEU reg (Initialize) }\end{array}$ \\
\hline 55 & L1A & $\mathrm{D}$ & $\mathrm{I}$ & $0 / 2.5$ & Yes* & Level 1 Accept \\
\hline 56 & PIPERD2 & $\mathrm{D}$ & $\mathrm{I}$ & $0 / 2.5$ & Yes* & Pipeline Read 2 \\
\hline 57 & PIPERD1 & $\mathrm{D}$ & $\mathrm{I}$ & $0 / 2.5$ & Yes* & Pipeline Read 1 \\
\hline 58 & PARST & $\mathrm{D}$ & $\mathrm{I}$ & $0 / 2.5$ & Yes* & Preamp Reset \\
\hline 61 & PRIINB & Diff & $\mathrm{O}$ & $0-2.5$ & Yes & Priority In minus \\
\hline 62 & PRIIN & Diff & $\mathrm{O}$ & $0-2.5$ & Yes & Priority In plus \\
\hline 63 & TNBR & $\mathrm{D}$ & $\mathrm{I} / \mathrm{O}$ & $0 / 2.5$ & Yes & $\begin{array}{l}\text { Top Neighbor; open drain } \mathrm{w} / 2 \mathrm{k} \\
\text { internal pull-up }\end{array}$ \\
\hline $80-207$ & $\operatorname{In}<0-127>$ & A & I & 0.45 & Yes & Detector Inputs \\
\hline
\end{tabular}

Table 11 Pin listing for the SVX4 chip. 
The differential signals are LVDS - i.e. they are signals designed to be driven over a differential line of a characteristic impedance of $100 \mathrm{ohms}$ and must be terminated into $100 \mathrm{ohms}$. The voltage differential is nominally $350 \mathrm{mV}$, and the mean voltage is $1.25 \mathrm{~V}$ (i.e. the single ended voltage in the ideal situation should vary from 1.075 volts to 1.425 volts). LVDS signals can tolerate significant common mode noise $(0<$ Vsingle $<2.5 \mathrm{~V})$.

Power consumption is described in detail in Ref [9].

Operating Voltage:

Absolute Max. Voltage:

Operating Current: AVDD:

SVDD:

DVDD:

Operating Frequency:

ESD protection:
2.5 V nom., 2.25 V min., 2.7 Vmax., all supplies $3.5 \mathrm{~V}$ all supplies* $60 \mathrm{~mA}$ 22-160 mA Readout mode $20 \mathrm{~mA}$ Readout mode (readall) or $9.2 \mathrm{~mA}$ Digitize mode, plus 30mA Acquire mode FECLK: 7.6 MHz@20\% duty cycle BECLK: 25 MHz Readout mode, 56 MHz Digitize mode@ 40-50\% duty cycle for detector input pads, diode protection to AVDD/AGND; for all other pads, diode protection to $\mathrm{xVDD} / \mathrm{AGND}$ with active power supply clamps to AGND

* Could cause permanent analog performance degradation. TBD. 


\subsubsection{A Note Regarding Vcal}

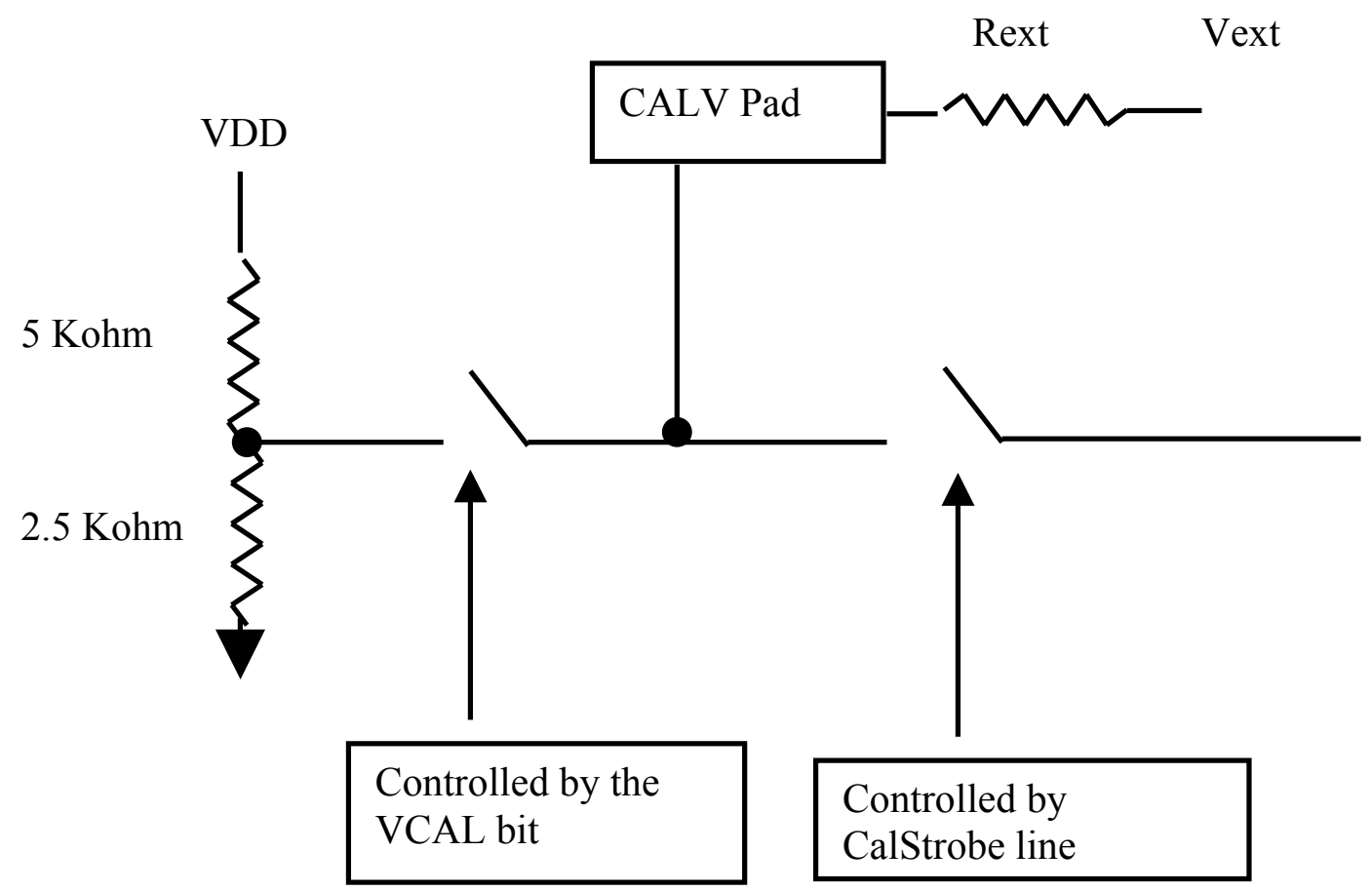

Figure 12 Cartoon schematic for the Vcal circuit in the SVX4 chip.

The voltage used to pulse the Cal Inject capacitors is derived either from the voltage applied to the CALV pad or from an internal voltage divider as shown in Figure 12. This arrangement, in the case of a multiplet of SVX4 chips supplied from a common external voltage via a shared resistor (Rext), allows for a 'poor mans way' of generating a variety of calibration voltages by turning on and off the VCAL bits for some of the SVX 4 chips without the use of a variable external voltage!

\subsection{External Components and Power}

In Figure 13, we show a cartoon schematic for the proper biasing of the SVX4 chip. 


\section{SVX4A EXTERNAL COMPONENTS AND POWER 4/3/02}

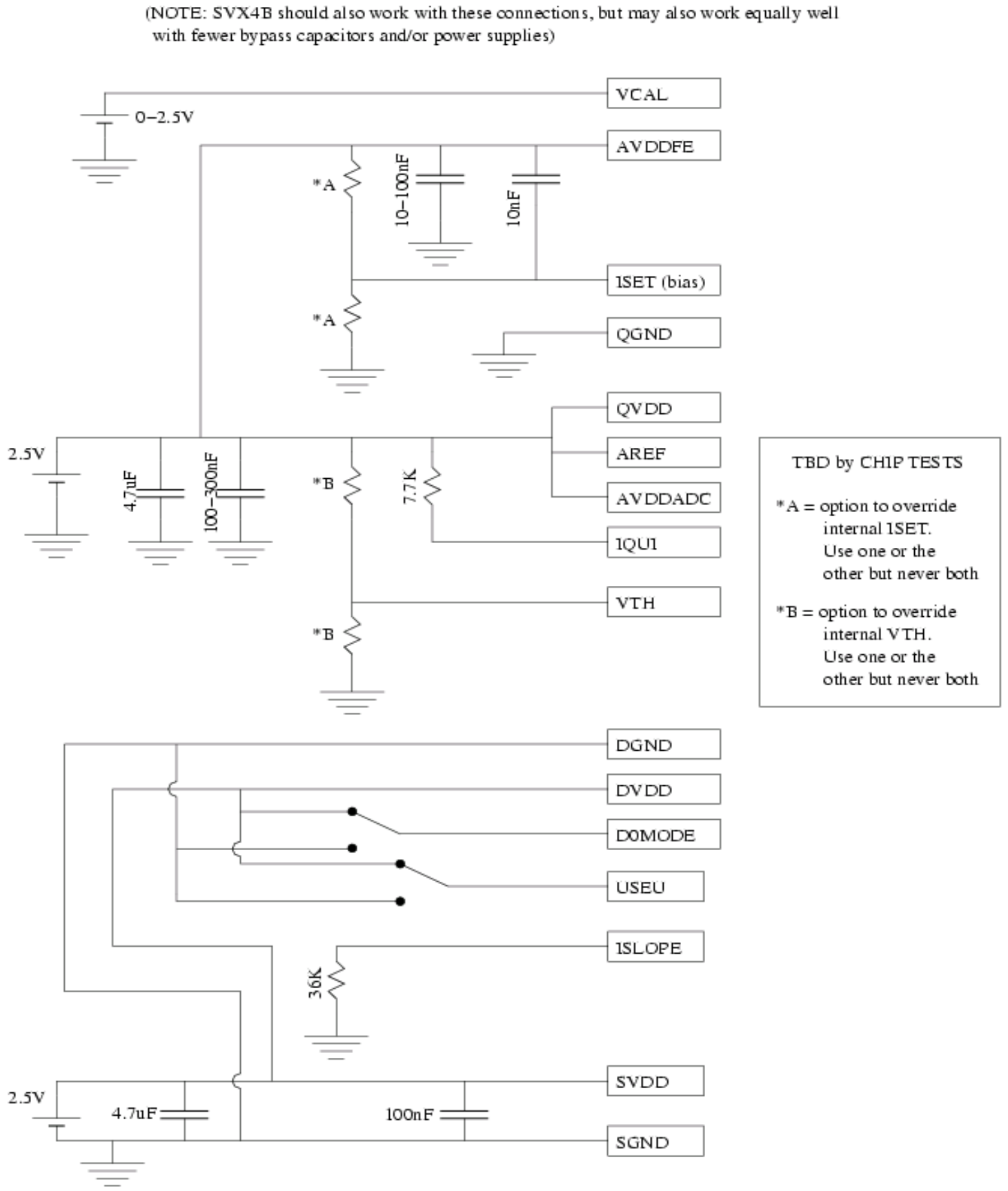

Figure 13 Diagram showing the external components that are needed for proper biasing of the SVX4 chip. 


\section{Operating the Chip}

The SVX4 chip is a monolithic chip incorporating a "front-end" section and a "back-end". In earlier versions these were on separate chips, and it is still common practice to refer to the "front-end" and "back-end" areas. Thus, the following nomenclature is used:

- $\mathrm{FE} \rightarrow$ "FE" is for "front-end". This area has the analog amplifier, pipeline, and deadtimeless skip-logic (or pipeline logic).

- $\mathrm{BE} \rightarrow$ "BE" is for "back-end". This area has the digitization, FIFO and readout logic.

- Hybrid $\rightarrow$ the ceramic circuit layout that holds 2 to 10 SVX4 chips and services either r- $\phi$ side of the r-z side of the silicon sensors.

\subsection{Guide for Single Chip Operation}

- Ideally there should be one very large bypass capacitor (greater the $1 \mu \mathrm{F}$ ) per hybrid for AVDD and DVDD.

- There should be one common ground plane for the chip sets on the hybrid. An exposed section of this ground plane is where the back face of the SVX4 chip rests. The digital power feed should have its own return line, connected to the ground plane at one point per hybrid.

- IQUISCIENT should be biased with a $7.7 \mathrm{k} \Omega$ resistor to AVDD. ISLOPE should be biased with a $\mathrm{k} \Omega$ resistor.

- FECLK and BECLK should be wired bonded together for $\mathrm{D} \varnothing$ mode operation.

\subsection{Guide for Daisy Chain Operation}

The SVX4 is designed for daisy chained operation to minimize the number of bus and control lines required to operate the device. Fewer control lines means less space on the high density interconnect and less mass in the system. A group of daisy chained chips is shown in Figure 14. All the chips share a common communication bus (BUS0-7) and a common differential clock (FE-CLK, BE-CLK). 


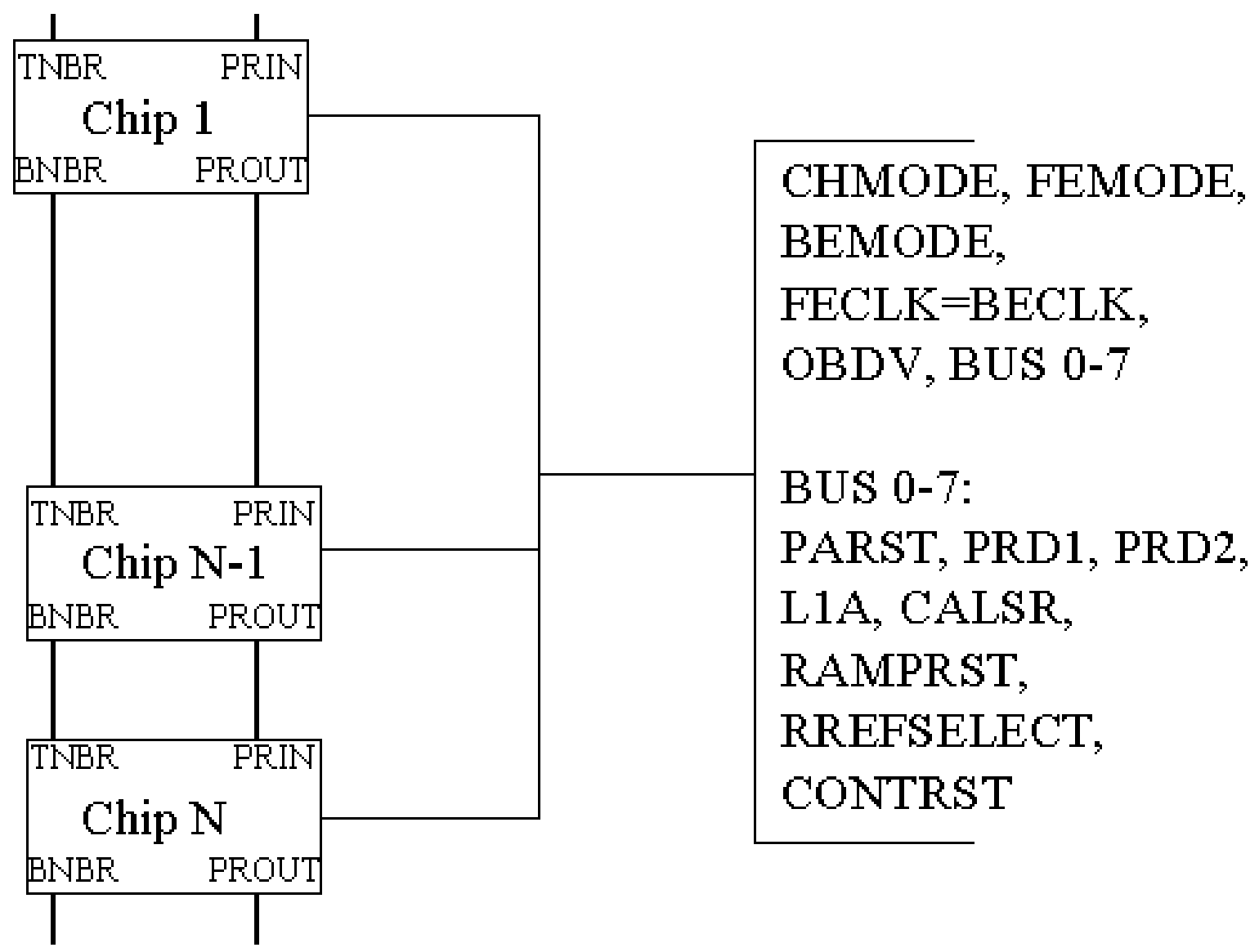

Figure 14 Daisy chained readout chips.

In addition, each chip has two pads call TNBR and BNBR that are used for communication between adjoining chips. After powering up the SVX4, the chip parameters listed in Section 3 must be downloaded before useful operation of the readout chips can begin. For each SVX4 chip, 192 bits must be downloaded into internal registers. In the Initialization Cycle, the signal lines PRIIN and PRIOUT are used as a serial data link to form a very long shift register for downloading parameters to the string of daisy chained SVX4s. Parameters for each chip are loaded in sequential order with the data for chip 1 loaded first via PRIOUT on the last chip of the daisy chain. Data is clocked between cells in the shift register using the common differential clock pads. If there were 10 chips, exactly 1920 bits would have to be downloaded in the Initialize Cycle. Downloaded parameters may be checked by shifting the bits out through PRIIN of the first chip while reloading the chips with the same data. To identify each chip in the daisy chain, a separate chip ID number (bits 150-156) is downloaded into each chip during the Initialize Cycle. The seven bit chip ID number allows chips to be tagged with numbers from 0 to 127.

In Acquire Cycle, the Bus0-7 and clock pads provide simultaneous real time control of all the chips in the daisy chain. PRININ, PRIOUT, TNBR, and BNBR have no function in Acquire Cycle. 
In Digitize Cycle, the Bus0-7 and differential clock pads provide real time control of all the chips in the daisy chain. The TNBR and BNBR pads are used only in READ NEIGHBOR mode to notify the corresponding chip to read out the extremum strip. The pads PRIIN and PRIOUT are used to pass a token in between chips to control when each chip should put data onto the bus and when readout is complete.

In Readout Cycle, the Bus 0-7 lines are changed from input lines to output lines. During readout, data from each SVX4 chip is placed on the common bus beginning with the first chip in the daisy chain and proceeding sequentially through the remaining chips. Information is placed on the bus in 8 bit bytes. First the chip ID and then the pipeline cell is read out. Then the address and data information for that chip is read out beginning with the channel nearest the top of the chip, channel 1, and proceeding downward. Priority for the output is passed from the PRIOUT of the first chip to the PRIIN pad of the next chip after the first chip has been completely readout. The top chip will have PRIIN first since the PRIIN pad is internally pulled weakly low to initiate readout. Information is readout using both the high and low transitions of the differential clock. Thus, the channel readout rate is approximately equal to the BECLK clock frequency.

\section{Appendix A}

\subsection{Measurements of Timing on Various Test Stands}

\subsubsection{Systems in use}

- The Stimulus Test Stand. This system is based on a general pattern generator that is quite expensive and rather delicate to program. It is however an off-the-shelf item. It has not been setup is any triggerable manner.

- The Stand Alone Test Chain. This used the full test chain for $\mathrm{D} \varnothing$.

- The PATT Test Stand. This test stand was used at LBL and the wafer probing station. It uses a text file to download the initialization stream.

\subsection{Measurements of the Basic Sequence}

Analysis of the basic sequence of signals going to and coming from the SVX4 involves studying the Digitization and Readout Cycles while Acquisition is occurring. Items of interest to observers are the changes that occur at each of the cycle boundaries, the acceptance of a trigger and its handling, and the return of the pipeline cell after readout. For the systems that do not run continuous sequences, it is useful to examine the start and end of the sequence chain and to compare the state of the various lines before the start and after the end of a sequence burst.

This suggests the following measurements be made:

- Basic Clock Rate. A measurement of the clock speed used to drive the sequence of signals going to/coming from the chip is useful in each of the systems. Systems may be able to only change states at a one-half their basic clock rates. This has consequences for the rate at which the FECLK or BECLK may be run in comparison to basic clock rate.

- Full Sequence. A snapshot of the full sequence starting with the beginning of the burst of signals going to the SVX4 and ending with the end of the burst gives an overview of which lines make transitions at which points. Detailed timing relationships cannot be easily observed. 
- Preamp Reset. The reset of the preamp should occur between bunches. This is handled differently on various systems and may affect the results they produce. Measurements of where these transitions occur have been made.

- Start of Sequence. The disposition of the various signal lines at the start of the sequence is relevant for systems that do not run continuously.

- Level 1 Accept. The level 1 accept causes the pipeline cell to be put aside.

- Digitization. Resetting of the Wilkinson ramp, the counter, the threshold comparators and the interaction with the PRD1 signals that place the pedestal and then the signal capacitor in place for measurement are critical portions of the SVX4 operation and hence are interesting to measure.

- End of Digitization. The signaling of the end of the digitization and the observation of the operation of the top and bottom neighbor logic in action are interesting to document.

- End of Digitization/Beginning of Readout. The transition from the end of the digitization to the readout, noting the modification of the BEMODE line over this boundary is of interest to document.

- Beginning of Readout. Examination of the data lines and their correlations with data that are eventually seen in computer memory of the device used to store that data are interesting to study.

- End of Readout. The final data words, together with observation of how the data stream to the computer is terminated, are correlated with the data as they appear on the computer.

- End of Sequence. The end of the commands sent to the chip for systems that send only bursts of sequences are useful measurements so that the quiescent state of the lines between bursts can be studied.

\subsection{The DO Sequence}

The following pictures show the simulation waveforms that were used to test the schematic level design of the SVX4. Both D0 and CDF agreed to choose waveforms that would test most of the features of the SVX4 and to be complementary to each other. One waveform tests one set of features and the other waveform tests orthogonal features. The parameters used for the D0 sequence were as follows:

- Injection mask: 125 injected on only

- Mask disable is off

- Bandwidth settings: 0000

- Bias controls: 0010

- Trigger latency: 13

- Pipeline readout order: signal cell first, then pedestal cell 47

- Chip id: 0

- DPS setting: on

- Channel 127 readout: not read out

- Channel 63 readout: read out

- Sparsify mode: on

- Read neighbors: on

- Ramp pedestal: 0000

- Ramp direction, comparator polarity: both 0

- Ramp range: 000

- Threshold: 20 
- Counter modulo: 255

- Note that 46 cycles before the first operation are used to assure that all pipeline cells have valid data (thus the first L1 is issued at the $59^{\text {th }}$ clock pulse (i.e. $46+13$ for trigger latency)

- WARNING : These waveforms were generated and used during the simulation phase of the design of the chip. Similar waveforms have been used for operational testing of the chip but with some significant differences : In the waveforms the CHMODE line is not properly used, for maximum noise immunity this line should be high only during the period that the BEMODE and FEMODE lines are changing. In addition the PRIOUT line during initialization will reflect whatever random state the download register is in after power up (in these simulations that state was set to be the same state as the one we end up in). PRIOUT during initialization will have well defined data only after the chip has been fully loaded, as described earlier in the section where the daisy chaining of chips was discussed.

In Figure 15, we show the full $\mathrm{D} \varnothing$ waveform with the sparsified readout option active. In Figure 16, we show the full $\mathrm{D} \varnothing$ waveform expanded to show the initialization cycle. In Figure 17, we show the full $\mathrm{D} \varnothing$ waveform expanded to see the acquire cycle. In Figure 18 , we show the $\mathrm{D} \varnothing$ waveform expanded to see the digitization and readout cycles. 


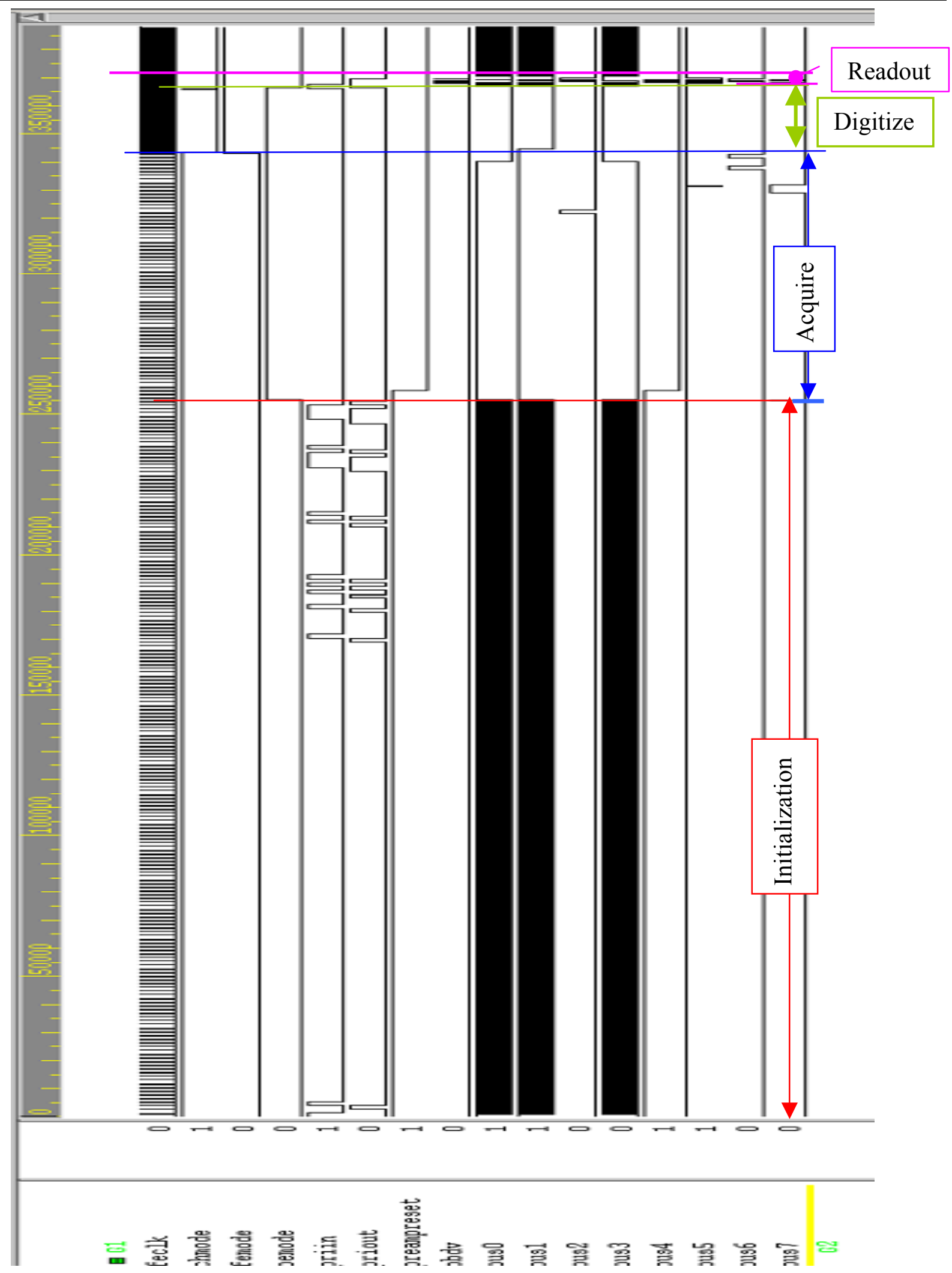

Figure 15 The full D0 waveform using a sparsified setting for readout. 


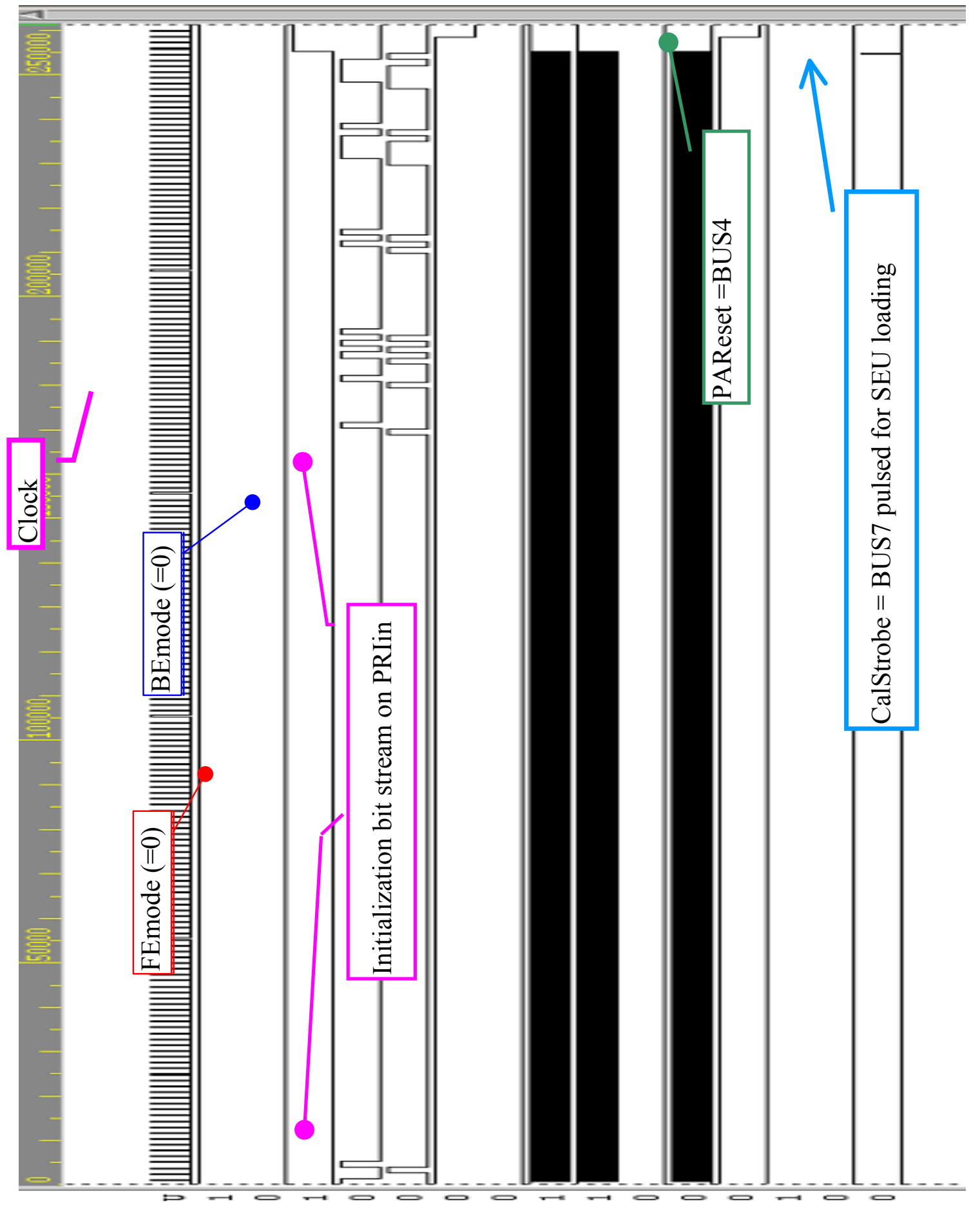

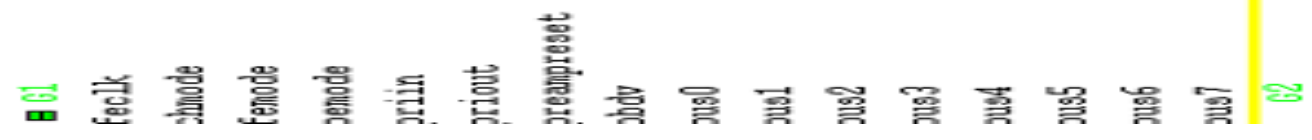

Figure 16 The initialization sequence for D0 mode operation. 


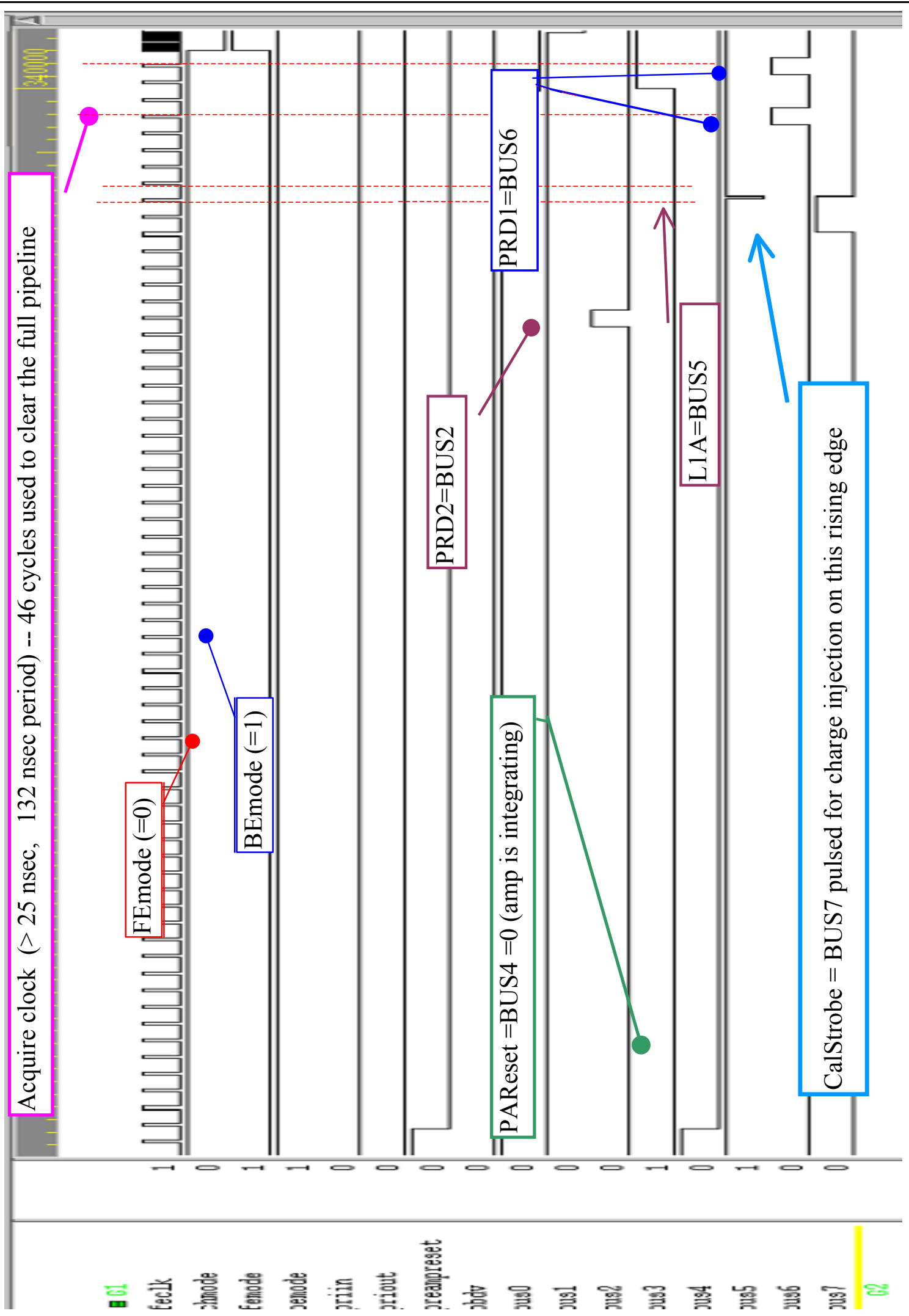

Figure 17 An enlargement of the acquire sequence in the front-end in D0 mode. 


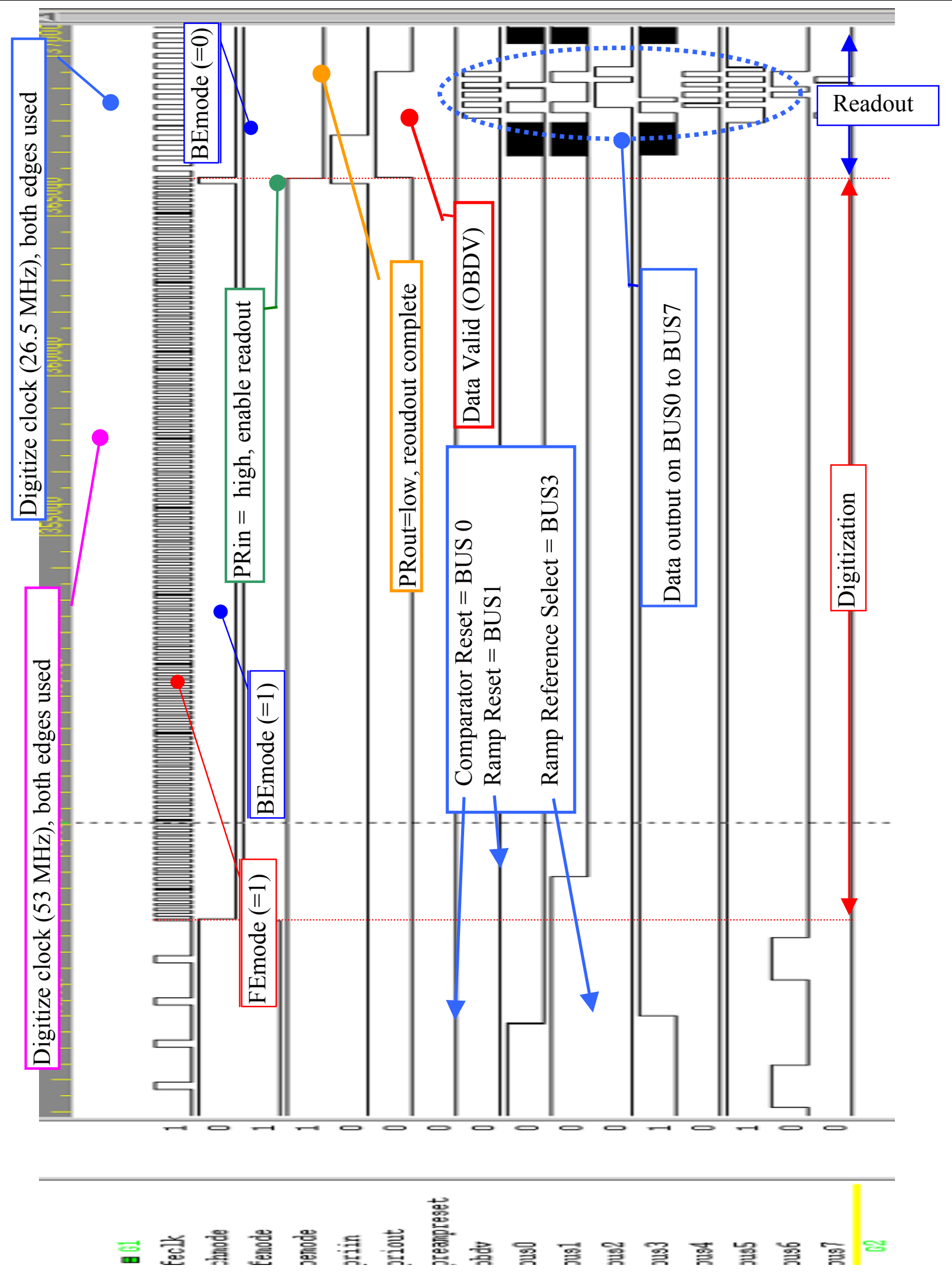

Figure 18 An enlargement of the digitization and readout in with data sparsification on in D0 mode. 


\subsection{The CDF Sequence}

The following pictures show the simulation waveforms that were used to test the schematic level design of the SVX4. Both D0 and CDF agreed to choose waveforms that would test most of the features of the SVX4 and to be complementary to each other. One waveform testing one set of features and the other waveform testing orthogonal features. The parameters used for the D0 sequence were as follows:

- Injection mask : every $8^{\text {th }}$ channel

- Mask disable is off

- Bandwidth settings: 1000

- Bias controls: 0010

- Trigger latency: 5

- Pipeline readout order: signal cell first, then pedestal cell 47

- Chip id: 24

- DPS setting: off

- Channel 127 readout: read out

- Channel 63 readout: not read out

- Sparsify mode: on

- Read neighbors: off

- Ramp pedestal: 1000

- Ramp direction, comparator polarity: both 0

- Ramp range: 000

- Threshold: 150

- Counter modulo: 240

- Note that 46 cycles before the first operation are used to assure that all pipeline cells have valid data (thus the first L1 is issued at the $51^{\text {st }}$ clock pulse (i.e. $46+5$ for trigger latency)

- WARNING : These waveforms were generated and used during the simulation phase of the design of the chip. Similar waveforms have been used for operational testing of the chip but with some significant differences: In the waveforms the CHMODE line is not properly used, for maximum noise immunity this line should be high only during the period that the BEMODE and FEMODE lines are changing. In addition the PRIOUT line during initialization will reflect whatever random state the download register is in after power up (in these simulations that state was set to be the same state as the one we end up in). PRIOUT during initialization will have well defined data only after the chip has been fully loaded, as described earlier in the section where the daisy chaining of chips was discussed.

In Figure 19, we show the full CDF waveform with the sparsified readout option active. In Figure 20, we show the full CDF waveform expanded to show the initialization cycle. In Figure 21, we show the full CDF waveform expanded to see the acquire cycle. In Figure 22, we show the CDF waveform expanded to see the digitization and readout cycles. 


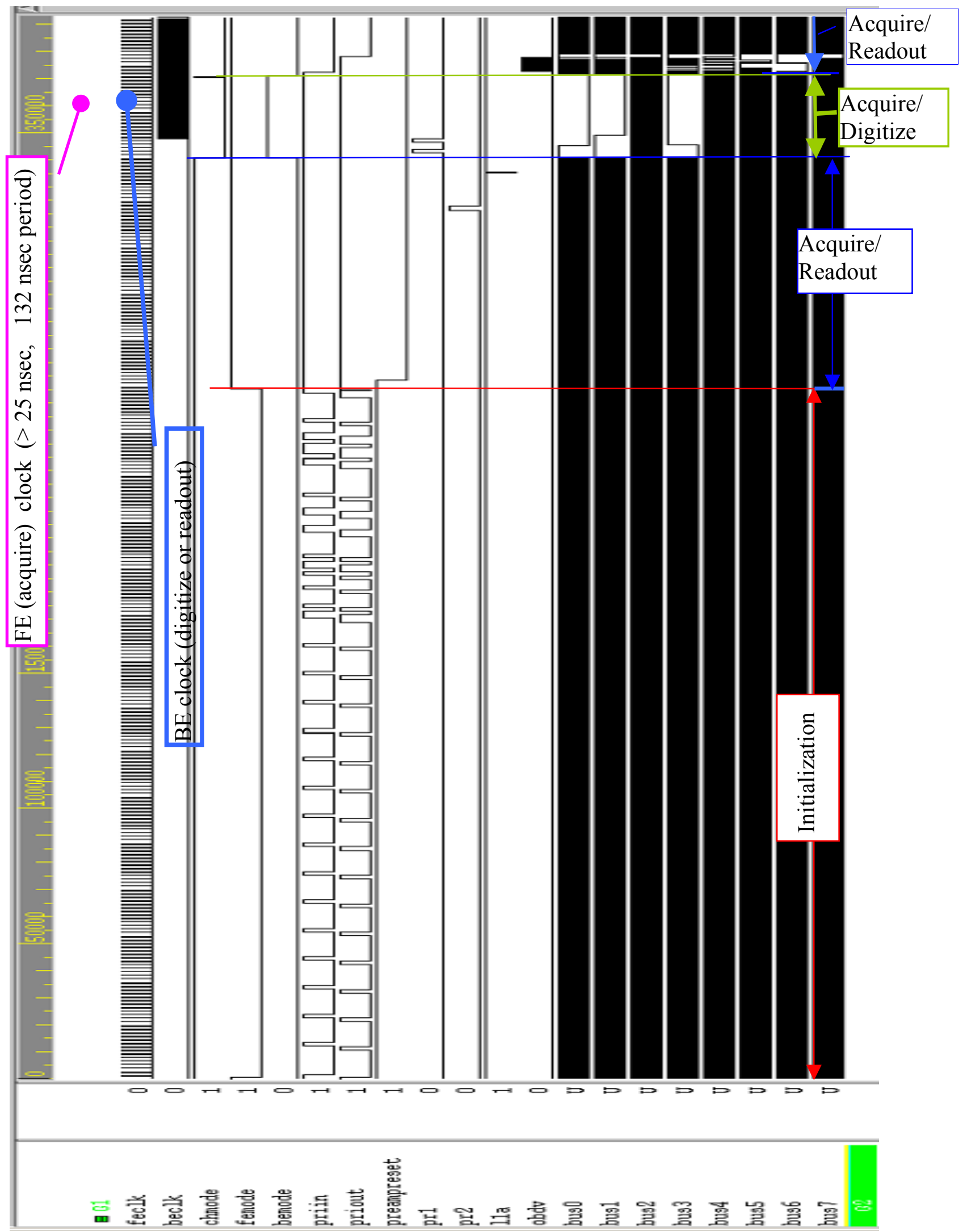

Figure 19 The full CDF sequence with data sparsification on. 


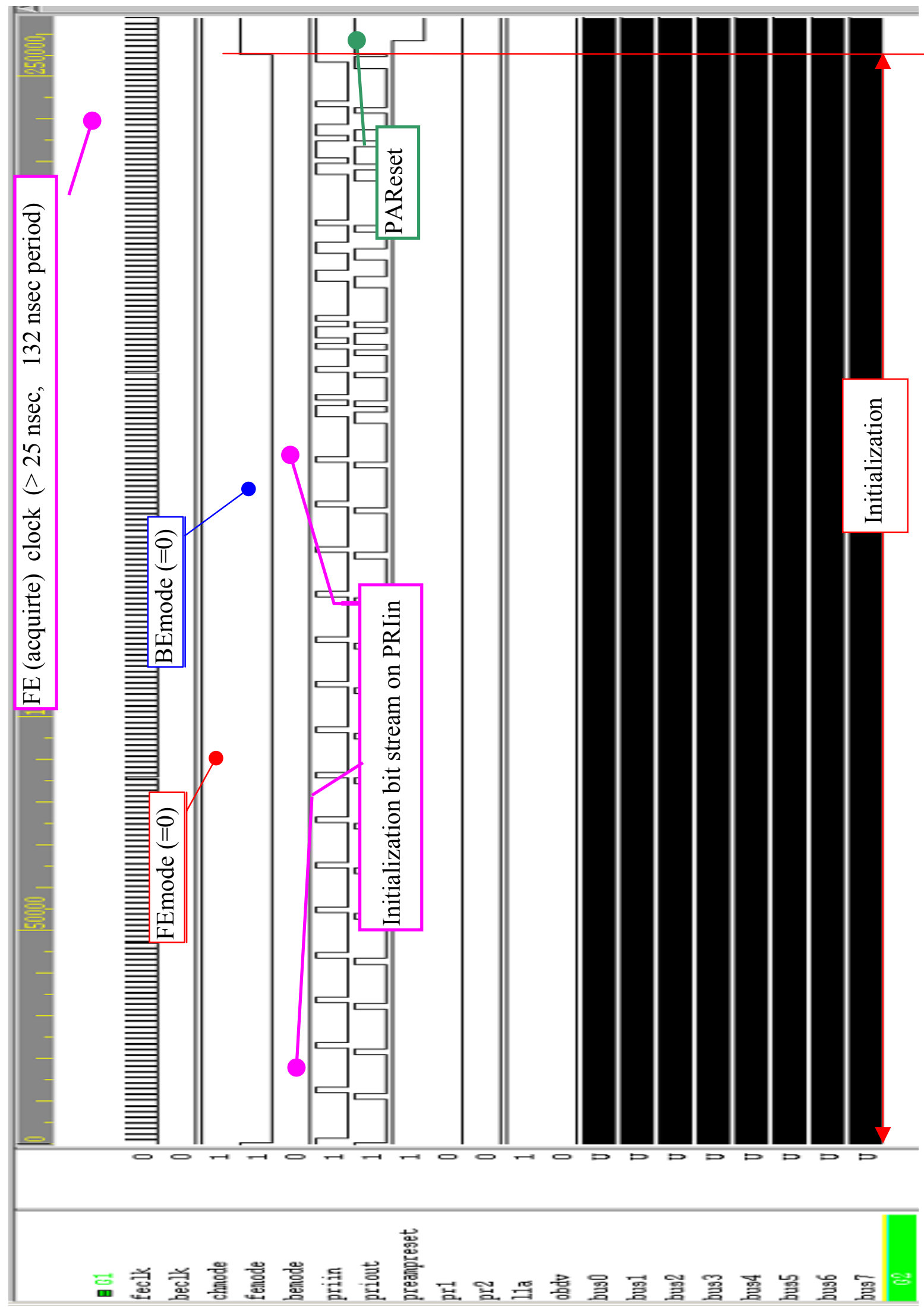

Figure 20 The initialization sequence for CDF mode. 


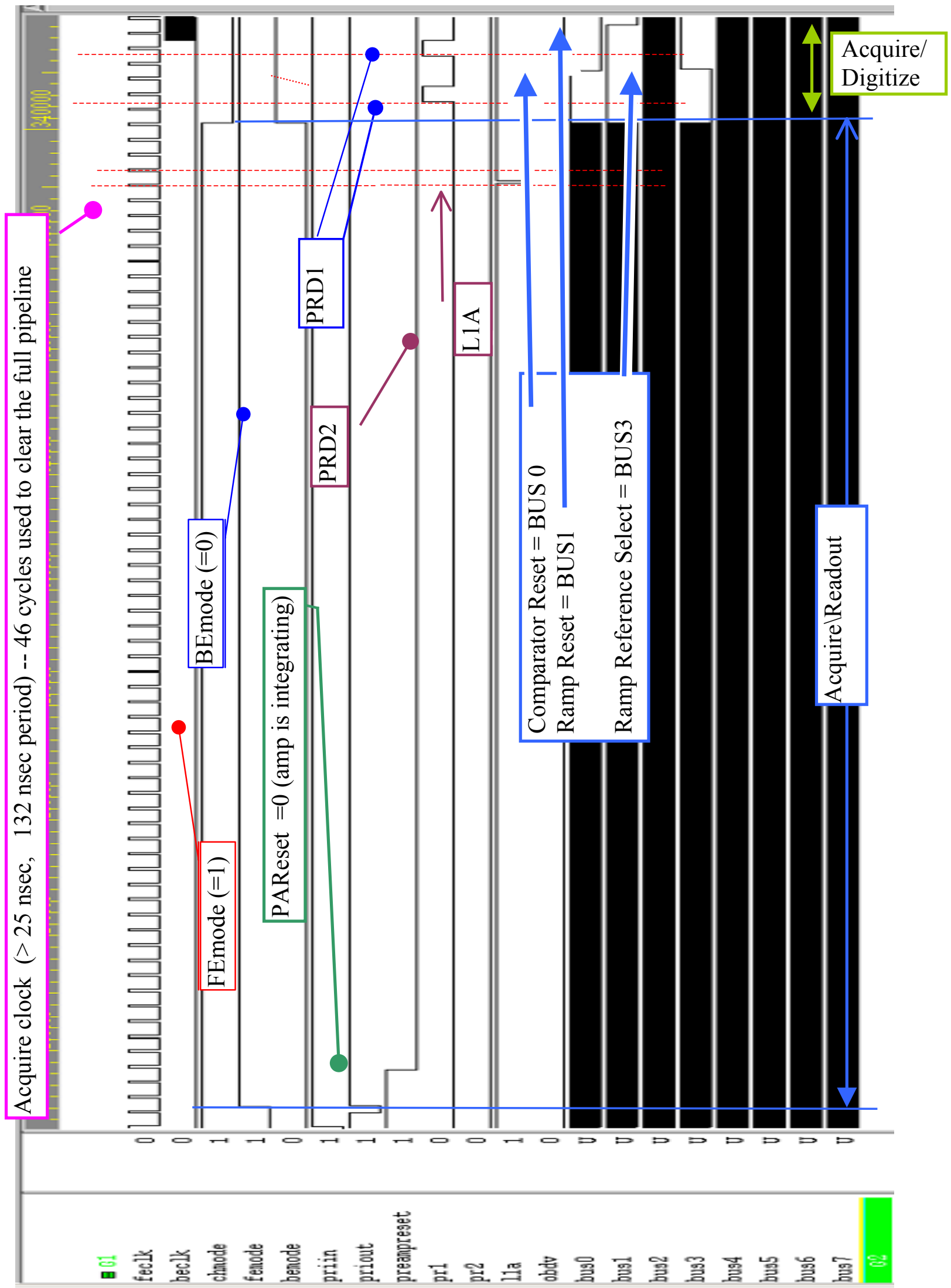

Figure 21 The acquire waveform for the CDF mode. 


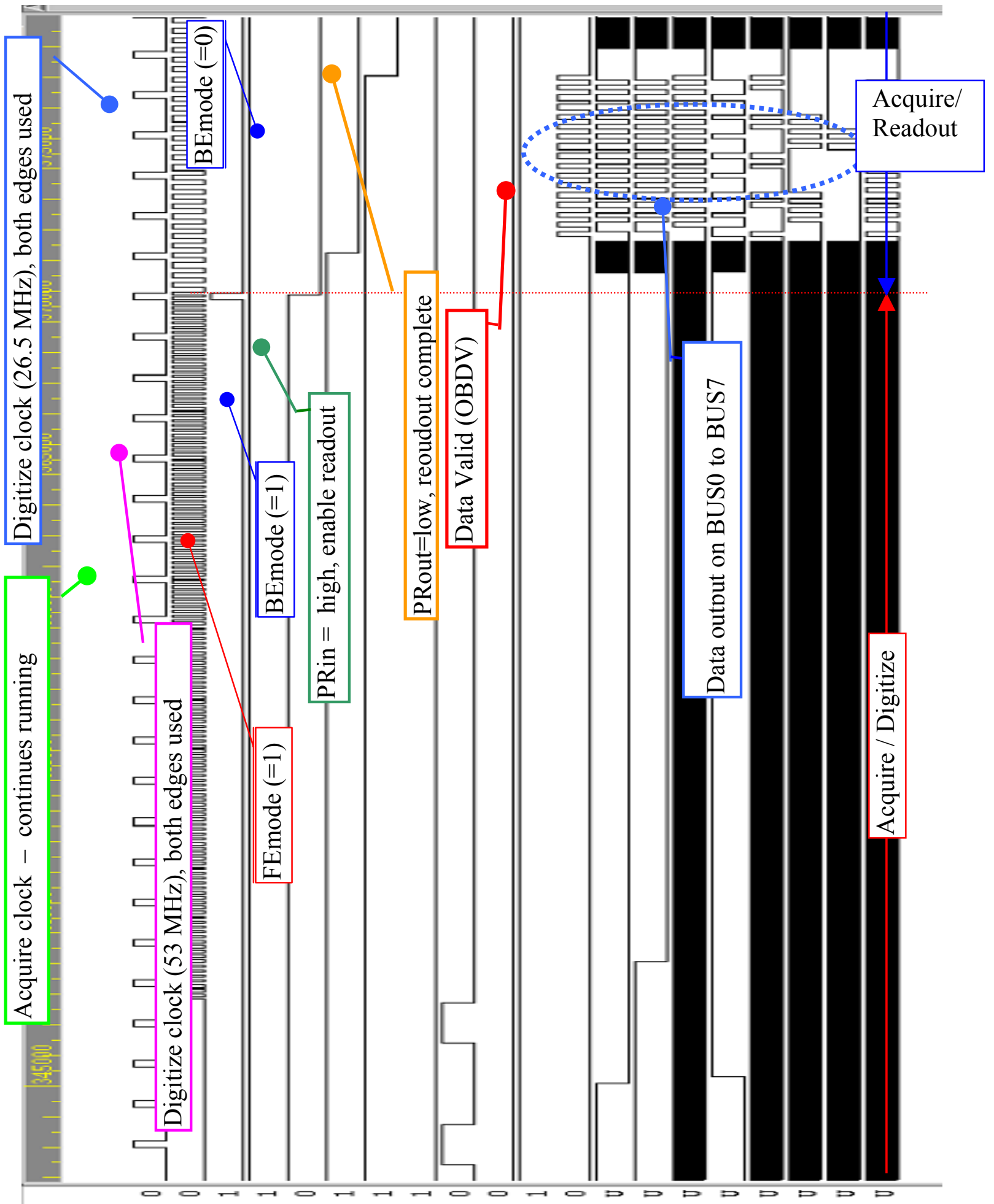

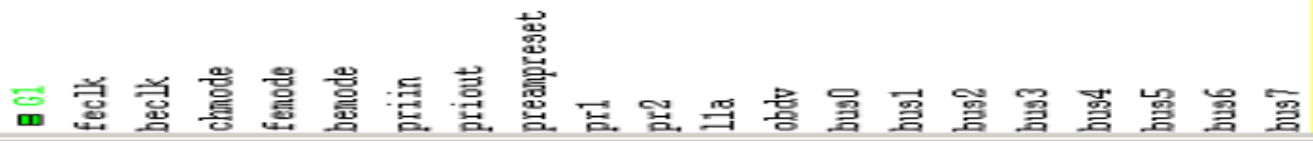

Figure 22 Digitization and readout for CDF mode. 


\section{Appendix B -The SVX4 Specifications (original list)}

This set of specifications was given to the chip designers as the design guideline. To the extent that there are changes between this early set of specs and the text of the rest of the manual - the text of the manual has the correct information.

\section{A. General:}

1. Input bonding pad pitch:

2. Overall Width:

3. Overall length:

4. Supply voltages:

5. Versions:

6. Bond pad layout:

7. Bond pads:

8. Maximum Supply Voltage:

B. Preamp:

1. Input pulse polarity:

2. Gain (feedback capacitor):

3. Gain uniformity (ch-to-ch) :

4. External load capacitance:

5. Risetime 0-90\%:

6. Risetime adjustment:

7. Noise (ENC) :

8. DC open loop gain:

9. Linearity:

10. Dynamic range:

11. Reset + settling time:

12. Reset offset voltage:

13. Input protection diodes:

14. Calibration injection:

15.Calibration charge control:

17. Input disable switch:
$48 \mu \mathrm{m}$

$6.250 \mathrm{~mm}$ active area. Dicing streets as close as allowed by design rules.

$<11.925 \mathrm{~mm}$

2.25-2.75V analog, 2.25-2.75V digital.

A version is the basic "conservative"

version.

B version adds on-chip bypassing and front to back combined power routing,

Both version have same bond pad layout with

some pads used only by CDF and others used only by D0.

Except Front End inputs, no wirebond pad is to be smaller than 150x150um (cover layer opening). Probe pads not meant for wirebonding are exempt.

$3.5 \mathrm{~V}$

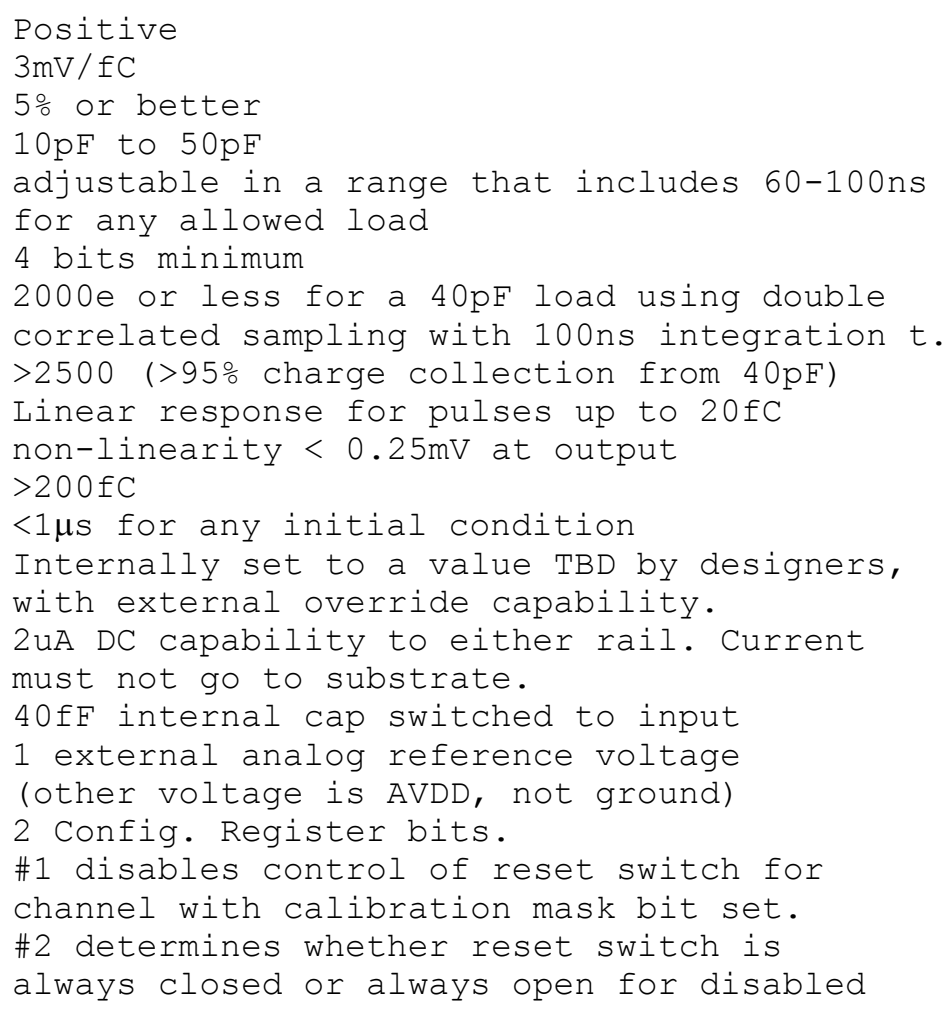


18. Input Device Current:

19. Bypass capacitors:

C. Pipeline:

1. Input Pulse polarity:

2. Voltage gain:

3. Gain uniformity:

4. Risetime, 0-90\%:

5. Noise (ENC at preamp input)

6. Linearity:

7. Dynamic Range:

8. Reset Time:

9. Pedestal uniformity:

10. Bias:

D. ADC:

1. Type:

2. Voltage Ramp:

3. Ramp rate "trim" bits:

4. Ramp Linearity:

5. Ramp dynamic range:

6. Ramp pedestal:

7. Counter:

8. Differential nonlinearity:

9. Bias:

E. Data output drivers:

1. Type:

2. Current source range:

3. Rise and fall times:

4. Common mode:

5: Load capability:

7. Tri-state:

8. Single ended use:

9. Bi-directional:

10. Output data skew: channels.

Adjustable with configuration bits as in SVX3 but with wider range (factor of 2). Performance in SVX-II mode should be maintained with no external bypass capacitors closer than $10 \mathrm{~mm}$.

Negative

3 to 5

5\% channel to channel

$10 \mathrm{~ns}$ to $40 \mathrm{~ns}$ (in that range, fixed)

$<500$ e

linear response up to $20 \mathrm{fC}$ at preamp input

To Be Confirmed: $>40 f C$ at preamp input

$<20$ ns for any allowed initial condition

$<500$ e at preamp input channel to channel

$<1000$ at preamp input cell to cell

Internally set with override bonding pad.

Wilkinson with real time pedestal

subtraction.

Rate adjustable with external resistor.

3 Bits, adding binary weighted capacitors to op-amp feedback. Largest capacitor is $4 x$ the fixed feedback capacitor. These capacitors provide a range adjustment- no fine adjustment needed.

$0.25 \%$ for rates between 0.1 and $1 \mathrm{~V} / \mathrm{us}$. $1 \mathrm{~V}$

Same as in SVX3.

8-bit Gray code, $106 \mathrm{MHz}$ rate.

$<0.5 \mathrm{LSB}$.

Internally set with override bonding pad

Complementary with "resistor current sources" $2.5 \mathrm{~mA}$ to $17.5 \mathrm{~mA}$ in $2.5 \mathrm{~mA}$ steps (3 bit

adjust).

$>2 \mathrm{~ns}$ and <4ns with nominal load.

VDD/2 nominal with $\mathrm{T}$ termination.

$70 \mathrm{ohm}$ and $20 \mathrm{pF}$.

Outputs tristated in initialize (except if SR copy pad is bonded- see H7) and digitize modes.

No additional requirements

All Bus pads will be bi-directional. Only some will be used of input as well as output by CDF, but all of them will be I/O for DO. $<3$ ns between OBDV and any bus line and between any two bus lines. 
F. TN/BN Pins:

1. Functions

2. Type, BN/TN:

3. Type, Priority in:

4. Type, Priority out:

5. BN/TN Internal pull-up:

6. BN/TN Pull-down current:

7. BN/TN Modes:

8. Priority in/out Modes:

9. Bonding pads:

G. Configuration Register:

1. Type:

2. Cell type:

3. Shadow register:

4. Clock:

5. Length:

6. Preset:

7. layout rule:

8. Bit order:

9. Bit Assignment:
The multiplexed functions of the SVX3 TN/BN pads will be separated in SVX4 to TN/BN and Priority in/out dedicated sets of pads. "Open collector" I/O with internal pull-up. Differential receiver (2 bond pads) same as clock receivers, with added high $Z$ common mode reference voltage (center tap of large resistance between power and ground). Differential driver (2 bond pads) same as data bus outputs.

$>500$ ohm

$>10 \mathrm{~mA}$

only active in digitize mode

Configuration register input/output during initialize mode. Priority passing during readout mode. Priority out high during digitize mode.

This increases the number of bonding pads per chip by 4 (2 next to $\mathrm{TN}$ and 2 next to $\mathrm{BN}$ ).

Bit serial shift register.

SEU tolerant shadow register.

Keep for SEU tolerance.

Register advanced with FE clock in initialize mode.

no limit.

no preset.

Do not place configuration register cells within $75 \mathrm{um}$ of a wirebond pad (they tend to be destroyed by missed wirebonds).

LSB loads first on all fields.

Numbers are for illustration. Designers may add bias adjust or other system bits as needed.

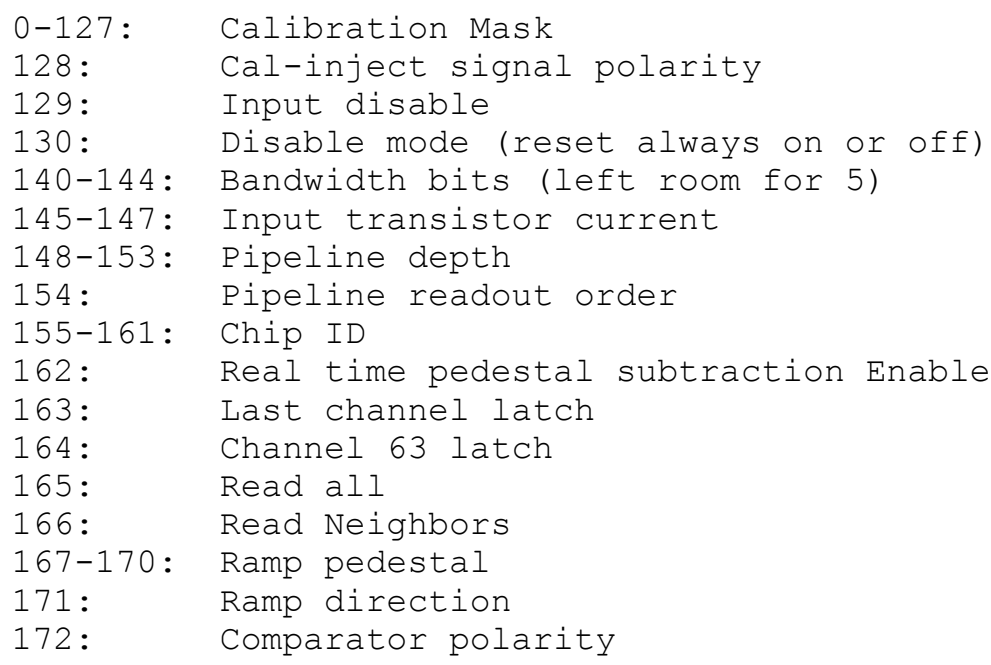


173-175: Ramp range selection

176-183: Sparsification threshold

184-191: Counter Modulo

192: $\quad$ First chip flag (see H.9)

193: Last chip flag (see H.9)

192-194: Output driver resistor select

H. Control Functions:

$(*)$ Denotes desirable feature but not strictly required

1. Signal Functions:

2. Ramp and Counter Reset:

3. Preamp Reset \& Fe Clock:

4. $\operatorname{PRD} 2(*)$ :

5. Last channel SR bit:

6. Chan. 63 latch SR bit (*):

7. Bus 3 SR copy bond pad:

8. extra L1A:

9. OBDV (data valid) control (*):

10. Readout Mode Pad:
All control signals same function as SVX3 except as noted here.

Remove Counter Reset as an independent signal. In normal mode Counter Reset is to be tied to Ramp Reset. In Dynamic Pedestal Subtraction mode Counter Reset is internally generated as in SVX3. Preamp Reset should always function independently of FE Clock state. In SVX3 Preamp Reset can only go high while FE Clock is high.

It is desirable that PRD2 control only the acquisition of the reference capacitor pedestal, and that the action of returning a cell to the pipeline be automatically triggered by the end of digitization (The falling edge of DIGITIZE MODE is used to drive the MOVE DATA pipeline input) on=always latch chan. 127 (same "last chip flag" in SVX3).

on=always latch chan. 63 (doubles read out speed) copy Priority out in initialize mode to Bus 3 output if pad is bonded to ground (and enable output bus in init. mode)

Additional L1A pulses (beyond 4) should be ignored by the pipeline logic.

OBDV must be driven by 1 chip per daisy chain at all times to prevent data transmission errors. This can be accomplished in SVX4 with 2 configuration register bits: First Chip (FC) and Last Chip (Different from item 5). OBDV control is given by the following logic table

$\begin{array}{cclll}\text { Pri. In } & \text { Pri. Out } & \text { FC } & \text { LC } & \text { OBDV } \\ \text { H } & \text { H } & \text { L } & \text { L } & \text { disabled } \\ \text { H } & \text { L } & \text { L } & \text { L } & \text { disabled } \\ \text { L } & \text { H } & \text { L } & \text { L } & \text { ENABLED } \\ \text { L } & \text { L } & \text { L } & \text { L } & \text { disabled* } \\ \text { X } & \text { H } & \text { H } & \text { L } & \text { ENABLED } \\ \text { X } & \text { L } & \text { H } & \text { L } & \text { disabled* } \\ \text { H } & \text { X } & \text { L } & \text { H } & \text { disabled } \\ \text { L } & \text { X } & \text { L } & \text { H } & \text { ENABLED }\end{array}$

*OBDV is to be disabled one BE CLOCK cycle after Pri. Out is lowered (same as in SVX3). [In the present CDF silicon system it was necessary to add logic to the port cards to implement this function, because the SVX3 does not have the FC and LC bits.]

Add an output pad to make the "Readout Mode" internal signal available 
SVX4 User's Manual

11. D0 Mode pad:

12. Test outputs:
$\mathrm{D} \varnothing$ Note 4252

A special bond pad, if left un-bonded will set the chip in D0 mode. This will multiplex I/O signals onto all Bus lines and gate the Pipeline clock off during digitize and readout operations. Buffered preamp and pipeline outputs for one channel, Comparator output for 1 channel, Ramp probe point, RTPS comparator buffered input And output- all as in SVX3. Additional probe points as needed to fully test performance. 


\section{Appendix C - Decimal/Gray tables}

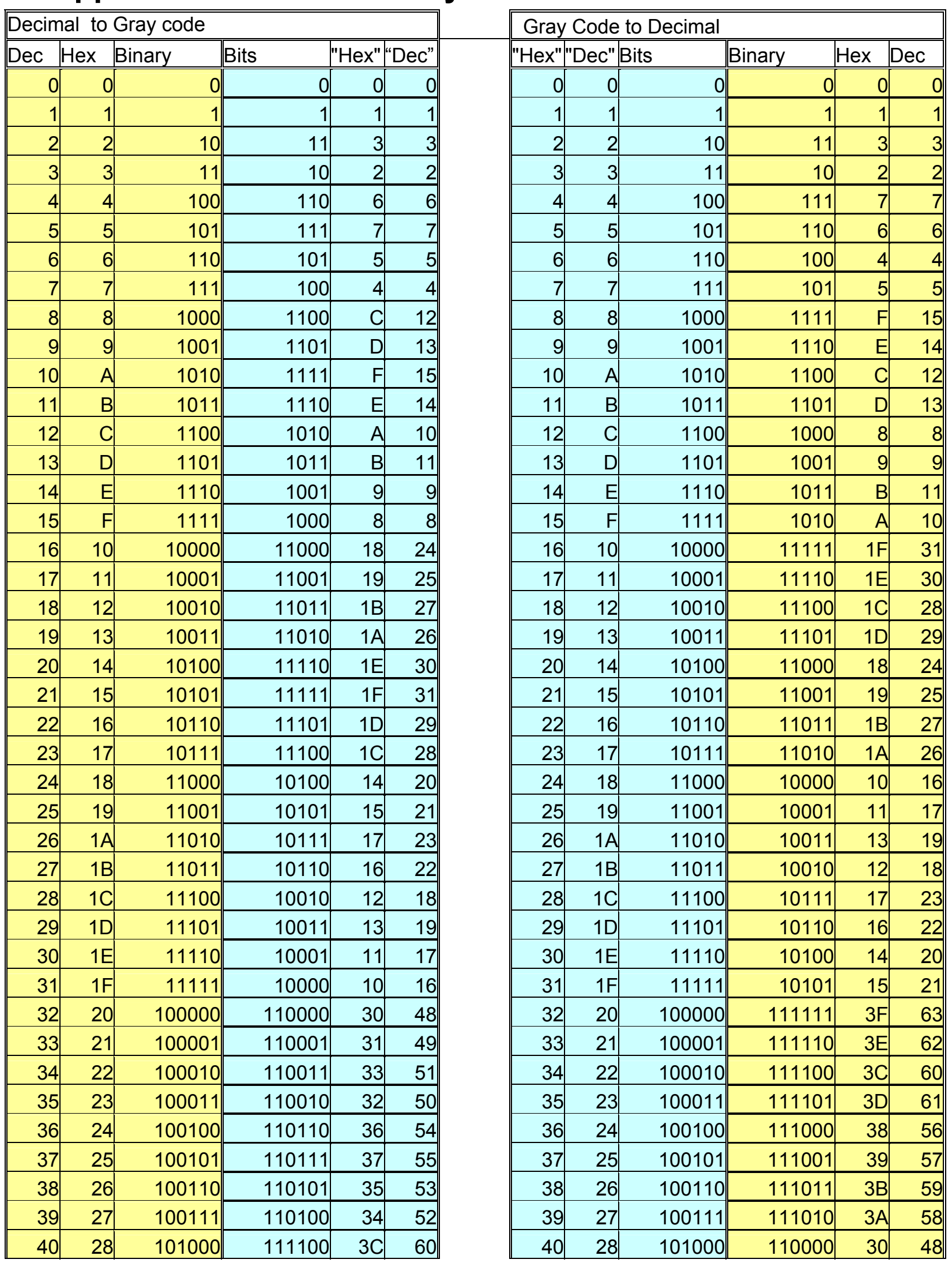


SVX4 User's Manual

D $\varnothing$ Note 4252

Decimal to Gray code

\begin{tabular}{|l|l|l||l|}
\hline Dec & Hex & Binary & Bits \\
\hline
\end{tabular} "Hex"|“Dec"

\begin{tabular}{|c|c|c|c|c|c|}
\hline 41 & 29 & 101001 & 111101 & & 61 \\
\hline 42 & $2 \mathrm{~A}$ & 101010 & 111111 & & 63 \\
\hline 43 & $2 \mathrm{~B}$ & 101011 & 111110 & $3 \mathrm{E}$ & 62 \\
\hline 44 & $2 \mathrm{C}$ & 01100 & 111010 & $3 A$ & 58 \\
\hline 45 & $2 \mathrm{D}$ & 101101 & 111011 & $3 B$ & 59 \\
\hline 46 & $2 \mathrm{E}$ & 101110 & 111001 & 39 & 57 \\
\hline 47 & $2 \mathrm{~F}$ & 101111 & 111000 & 38 & 56 \\
\hline 48 & 30 & 110000 & 101000 & 28 & 40 \\
\hline 49 & 31 & 110001 & 101001 & 29 & 41 \\
\hline 50 & 32 & 110010 & 101011 & $2 B$ & 43 \\
\hline 51 & 33 & 110011 & 1010 & $2 \mathrm{~A}$ & 42 \\
\hline 52 & 34 & 110100 & 101110 & $2 \mathrm{E}$ & 46 \\
\hline 53 & 35 & 110101 & 101111 & $2 \mathrm{~F}$ & 47 \\
\hline 54 & 36 & 110110 & 101 & $2 \mathrm{D}$ & 45 \\
\hline 55 & 37 & 110111 & 101100 & $2 \mathrm{C}$ & 44 \\
\hline 56 & 38 & & 100100 & 24 & 36 \\
\hline 57 & 39 & 11001 & 101 & 25 & 37 \\
\hline 58 & $3 A$ & 111010 & 100111 & 27 & 39 \\
\hline 59 & $3 \mathrm{~B}$ & 1011 & 110 & 26 & 38 \\
\hline 60 & $3 C$ & 111100 & 010 & 22 & 34 \\
\hline 61 & $3 \mathrm{D}$ & 111101 & 100011 & 23 & 35 \\
\hline 62 & $3 \mathrm{E}$ & 111110 & 001 & 21 & 33 \\
\hline 63 & $3 F$ & 111111 & 100000 & 20 & 32 \\
\hline 64 & 40 & 1000000 & 1100000 & 60 & 96 \\
\hline 65 & 41 & 001 & 110 & 61 & 97 \\
\hline 66 & 42 & 1000010 & 110 & 63 & 90 \\
\hline 67 & 43 & 1000011 & 1100010 & 62 & 98 \\
\hline 68 & 44 & & & 66 & 102 \\
\hline 69 & 45 & 1000101 & 1100111 & 67 & 103 \\
\hline 70 & 46 & 1000110 & 1100101 & 65 & 101 \\
\hline 71 & 47 & 1000111 & 100 & 64 & 100 \\
\hline 72 & 48 & 1001000 & 1101100 & $6 \mathrm{C}$ & 108 \\
\hline 73 & 49 & 1001001 & 101 & $6 \mathrm{D}$ & 109 \\
\hline 74 & $4 \mathrm{~A}$ & 1001010 & 111 & $6 \mathrm{~F}$ & 111 \\
\hline 75 & $4 \mathrm{~B}$ & 1001011 & 1101110 & $6 \mathrm{E}$ & 110 \\
\hline 76 & $4 C$ & 1001100 & 1101010 & $6 \mathrm{~A}$ & 106 \\
\hline 77 & $4 \mathrm{D}$ & 1001101 & 1101011 & $6 \mathrm{~B}$ & 107 \\
\hline 78 & $4 \mathrm{E}$ & 1001110 & 1101001 & 69 & 105 \\
\hline 79 & $4 \mathrm{~F}$ & 1001111 & 1101000 & 68 & 104 \\
\hline 80 & 50 & 1010000 & 1111000 & 78 & 120 \\
\hline 81 & 51 & 1010001 & 1111001 & 79 & 121 \\
\hline 82 & 52 & 1010010 & 1111011 & $7 \mathrm{~B}$ & 123 \\
\hline 83 & 53 & 1010011 & 1111010 & $7 \mathrm{~A}$ & 122 \\
\hline 84 & 54 & 1010100 & 1111110 & $7 \mathrm{E}$ & 12 \\
\hline
\end{tabular}

\begin{tabular}{|c|c|c|c|c|c|}
\hline \multicolumn{6}{|c|}{ Gray Code to Decimal } \\
\hline 'Hex"|' & "Dec" & & Binary & Hex & Dec \\
\hline 41 & 29 & 101001 & 110001 & 31 & 49 \\
\hline 42 & & 101010 & 110011 & 33 & 51 \\
\hline 43 & & 101011 & 110010 & 32 & 50 \\
\hline 44 & & 101100 & 110111 & 37 & 55 \\
\hline 45 & & 101101 & 110110 & 36 & 54 \\
\hline 46 & & 101110 & 110100 & 34 & 52 \\
\hline 47 & & 101111 & 110101 & 35 & 53 \\
\hline 48 & & 110000 & 100000 & 20 & 32 \\
\hline 49 & & 110001 & 100001 & 21 & 33 \\
\hline 50 & & 110010 & 100011 & 23 & 35 \\
\hline 51 & & 110011 & 100010 & 22 & 34 \\
\hline 52 & & 110100 & 100111 & 27 & 39 \\
\hline 53 & & 110101 & & 26 & 38 \\
\hline 54 & 36 & 110110 & 100100 & 24 & 36 \\
\hline 55 & 37 & 110111 & 100101 & 25 & 37 \\
\hline 56 & 38 & 111000 & & $2 \mathrm{~F}$ & 47 \\
\hline 57 & 39 & 111001 & 101110 & $2 \mathrm{E}$ & 46 \\
\hline 58 & $3 A$ & 111010 & 101100 & $2 \mathrm{C}$ & 44 \\
\hline 59 & $3 \mathrm{~B}$ & 111011 & & $2 \mathrm{D}$ & 45 \\
\hline 60 & $3 C$ & 111100 & 101000 & 28 & 40 \\
\hline 61 & $3 \mathrm{D}$ & 101 & 001 & 29 & 41 \\
\hline 62 & $3 \mathrm{E}$ & 111110 & 11 & $2 \mathrm{~B}$ & 43 \\
\hline 63 & $3 F$ & 111111 & 101010 & $2 \mathrm{~A}$ & 42 \\
\hline 64 & 40 & 1000000 & 111 & $7 F$ & 127 \\
\hline 65 & 41 & 1000001 & 1111110 & $7 \mathrm{E}$ & 126 \\
\hline 66 & 42 & 1000010 & 1111100 & $7 \mathrm{C}$ & 124 \\
\hline 67 & 43 & 1000011 & 11 & $7 \mathrm{D}$ & 125 \\
\hline 68 & 44 & 1000 & 00 & 78 & 120 \\
\hline 69 & 45 & 1000101 & 1111001 & 79 & 12 \\
\hline 70 & 46 & 1000110 & & $7 \mathrm{~B}$ & 123 \\
\hline 71 & 47 & 1000111 & 1111010 & $7 \mathrm{~A}$ & 122 \\
\hline 72 & 48 & 1001000 & 1110000 & 70 & 112 \\
\hline 73 & 49 & & 1110001 & 71 & 113 \\
\hline 74 & $4 \mathrm{~A}$ & 1001010 & 1110011 & 73 & 115 \\
\hline 75 & $4 \mathrm{~B}$ & 1001011 & 1110010 & 72 & 114 \\
\hline 76 & $4 C$ & 1001100 & 1110111 & 77 & 110 \\
\hline 77 & $4 \mathrm{D}$ & 1001101 & 1110110 & 76 & 118 \\
\hline 78 & $4 \mathrm{E}$ & 1001110 & 1110100 & 74 & 116 \\
\hline 79 & $4 \mathrm{~F}$ & 1001111 & 1110101 & 75 & 117 \\
\hline 80 & 50 & 1010000 & 1100000 & 60 & 96 \\
\hline 81 & 51 & 1010001 & 1100001 & 61 & 97 \\
\hline 82 & 52 & 1010010 & 1100011 & 63 & 99 \\
\hline 83 & 53 & 1010011 & 1100010 & 62 & 98 \\
\hline 84 & 54 & 1010100 & 1100111 & 67 & 103 \\
\hline
\end{tabular}


SVX4 User's Manual

D $\varnothing$ Note 4252

Decimal to Gray code

\begin{tabular}{|l|l|l||l|}
\hline Dec & Hex & Binary & Bits \\
\hline
\end{tabular}

"Hex"|“Dec"

\begin{tabular}{|r|r|r|r|r|r|}
\hline \hline 85 & 55 & 1010101 & 1111111 & $7 \mathrm{~F}$ & 127 \\
\hline 86 & 56 & 1010110 & 1111101 & $7 \mathrm{D}$ & 125 \\
\hline 87 & 57 & 1010111 & 1111100 & $7 \mathrm{C}$ & 124 \\
\hline 88 & 58 & 1011000 & 1110100 & 74 & 116 \\
\hline 89 & 59 & 1011001 & 1110101 & 75 & 117 \\
\hline 90 & $5 \mathrm{~A}$ & 1011010 & 1110111 & 77 & 119 \\
\hline 91 & $5 \mathrm{~B}$ & 1011011 & 1110110 & 76 & 118 \\
\hline 92 & $5 \mathrm{C}$ & 1011100 & 1110010 & 72 & 114 \\
\hline 93 & $5 \mathrm{D}$ & 1011101 & 1110011 & 73 & 115 \\
\hline 94 & $5 \mathrm{E}$ & 1011110 & 1110001 & 71 & 113 \\
\hline 95 & $5 \mathrm{~F}$ & 1011111 & 1110000 & 70 & 112 \\
\hline 96 & 60 & 1100000 & 1010000 & 50 & 80 \\
\hline 97 & 61 & 1100001 & 1010001 & 51 & 81 \\
\hline 98 & 62 & 1100010 & 1010011 & 53 & 83 \\
\hline 99 & 63 & 1100011 & 1010010 & 52 & 82 \\
\hline 100 & 64 & 1100100 & 1010110 & 56 & 86 \\
\hline 101 & 65 & 1100101 & 1010111 & 57 & 87 \\
\hline 102 & 66 & 1100110 & 1010101 & 55 & 85 \\
\hline 103 & 67 & 1100111 & 1010100 & 54 & 84 \\
\hline 104 & 68 & 1101000 & 1011100 & $5 \mathrm{C}$ & 92 \\
\hline 105 & 69 & 1101001 & 1011101 & $5 \mathrm{D}$ & 93 \\
\hline 106 & $6 \mathrm{~A}$ & 1101010 & 1011111 & $5 \mathrm{~F}$ & 95 \\
\hline 107 & $6 \mathrm{~B}$ & 1101011 & 1011110 & $5 \mathrm{E}$ & 94 \\
\hline 108 & $6 \mathrm{C}$ & 1101100 & 1011010 & $5 \mathrm{~A}$ & 90 \\
\hline 109 & $6 \mathrm{D}$ & 1101101 & 1011011 & $5 \mathrm{~B}$ & 91 \\
\hline 110 & $6 \mathrm{E}$ & 1101110 & 1011001 & 59 & 89 \\
\hline 111 & $6 \mathrm{~F}$ & 1101111 & 1011000 & 58 & 88 \\
\hline 112 & 70 & 1110000 & 1001000 & 48 & 72 \\
\hline 113 & 71 & 1110001 & 1001001 & 49 & 73 \\
\hline 114 & 72 & 1110010 & 1001011 & $4 \mathrm{~B}$ & 75 \\
\hline 115 & 73 & 1110011 & 1001010 & $4 \mathrm{~A}$ & 74 \\
\hline 116 & 74 & 1110100 & 1001110 & $4 \mathrm{E}$ & 78 \\
\hline 117 & 75 & 1110101 & 1001111 & $4 \mathrm{~F}$ & 79 \\
\hline 118 & 76 & 1110110 & 1001101 & $4 \mathrm{D}$ & 77 \\
\hline 119 & 77 & 1110111 & 1001100 & $4 \mathrm{C}$ & 76 \\
\hline 120 & 78 & 1111000 & 1000100 & 44 & 68 \\
\hline 121 & 79 & 1111001 & 1000101 & 45 & 69 \\
\hline 122 & $7 \mathrm{~A}$ & 1111010 & 1000111 & 47 & 71 \\
\hline 123 & $7 \mathrm{~B}$ & 1111011 & 1000110 & 46 & 70 \\
\hline 124 & $7 \mathrm{C}$ & 1111100 & 1000010 & 42 & 66 \\
\hline 125 & $7 \mathrm{D}$ & 1111101 & 1000011 & 43 & 67 \\
\hline 126 & $7 \mathrm{E}$ & 1111110 & 1000001 & 41 & 65 \\
\hline 127 & $7 \mathrm{~F}$ & 1111111 & 1000000 & 40 & 64 \\
\hline 128 & 80 & 10000000 & 11000000 & $\mathrm{C0}$ & 192 \\
\hline & & & & & \\
\hline
\end{tabular}

\begin{tabular}{|r|r|r|r|r|r||}
\hline \hline Gray Code to Decimal & \multicolumn{3}{|l|}{} \\
\hline \hline "Hex"|"Dec" & Bits & Binary & Hex & Dec \\
\hline 85 & 55 & 1010101 & 1100110 & 66 & 102 \\
\hline 86 & 56 & 1010110 & 1100100 & 64 & 100 \\
\hline 87 & 57 & 1010111 & 1100101 & 65 & 101 \\
\hline 88 & 58 & 1011000 & 1101111 & $6 \mathrm{~F}$ & 111 \\
\hline 89 & 59 & 1011001 & 1101110 & $6 \mathrm{E}$ & 110 \\
\hline 90 & $5 \mathrm{~A}$ & 1011010 & 1101100 & $6 \mathrm{C}$ & 108 \\
\hline 91 & $5 \mathrm{~B}$ & 1011011 & 1101101 & $6 \mathrm{D}$ & 109 \\
\hline 92 & $5 \mathrm{C}$ & 1011100 & 1101000 & 68 & 104 \\
\hline 93 & $5 \mathrm{D}$ & 1011101 & 1101001 & 69 & 105 \\
\hline 94 & $5 \mathrm{E}$ & 1011110 & 1101011 & $6 \mathrm{~B}$ & 107 \\
\hline 95 & $5 \mathrm{~F}$ & 1011111 & 1101010 & $6 \mathrm{~A}$ & 106 \\
\hline 96 & 60 & 1100000 & 1000000 & 40 & 64 \\
\hline 97 & 61 & 1100001 & 1000001 & 41 & 65 \\
\hline 98 & 62 & 1100010 & 1000011 & 43 & 67 \\
\hline 99 & 63 & 1100011 & 1000010 & 42 & 66 \\
\hline 100 & 64 & 1100100 & 1000111 & 47 & 71 \\
\hline 101 & 65 & 1100101 & 1000110 & 46 & 70 \\
\hline 102 & 66 & 1100110 & 1000100 & 44 & 68 \\
\hline 103 & 67 & 1100111 & 1000101 & 45 & 69 \\
\hline 104 & 68 & 1101000 & 1001111 & $4 \mathrm{~F}$ & 79 \\
\hline 105 & 69 & 1101001 & 1001110 & $4 \mathrm{E}$ & 78 \\
\hline 106 & $6 \mathrm{~A}$ & 1101010 & 1001100 & $4 \mathrm{C}$ & 76 \\
\hline 107 & $6 \mathrm{~B}$ & 1101011 & 1001101 & $4 \mathrm{D}$ & 77 \\
\hline 108 & $6 \mathrm{C}$ & 1101100 & 1001000 & 48 & 72 \\
\hline 109 & $6 \mathrm{D}$ & 1101101 & 1001001 & 49 & 73 \\
\hline 110 & $6 \mathrm{E}$ & 1101110 & 1001011 & $4 \mathrm{~B}$ & 75 \\
\hline 111 & $6 \mathrm{~F}$ & 1101111 & 1001010 & $4 \mathrm{~A}$ & 74 \\
\hline 112 & 70 & 1110000 & 1011111 & $5 \mathrm{~F}$ & 95 \\
\hline 113 & 71 & 1110001 & 1011110 & $5 \mathrm{E}$ & 94 \\
\hline 114 & 72 & 1110010 & 1011100 & $5 \mathrm{C}$ & 92 \\
\hline 115 & 73 & 1110011 & 1011101 & $5 \mathrm{D}$ & 93 \\
\hline 116 & 74 & 1110100 & 1011000 & 58 & 88 \\
\hline 117 & 75 & 1110101 & 1011001 & 59 & 89 \\
\hline 118 & 76 & 1110110 & 1011011 & $5 \mathrm{~B}$ & 91 \\
\hline 119 & 77 & 1110111 & 1011010 & $5 \mathrm{~A}$ & 90 \\
\hline 120 & 78 & 1111000 & 1010000 & 50 & 80 \\
\hline 121 & 79 & 1111001 & 1010001 & 51 & 81 \\
\hline 122 & $7 \mathrm{~A}$ & 1111010 & 1010011 & 53 & 83 \\
\hline 123 & $7 \mathrm{~B}$ & 1111011 & 1010010 & 52 & 82 \\
\hline 124 & $7 \mathrm{C}$ & 1111100 & 1010111 & 57 & 87 \\
\hline 125 & $7 \mathrm{D}$ & 1111101 & 1010110 & 56 & 86 \\
\hline 126 & $7 \mathrm{E}$ & 1111110 & 1010100 & 54 & 84 \\
\hline 127 & $7 \mathrm{~F}$ & 1111111 & 1010101 & 55 & 85 \\
\hline 128 & 80 & 10000000 & 11111111 & $\mathrm{FF}$ & 255 \\
\hline & & & & & \\
\hline
\end{tabular}


SVX4 User's Manual

D $\varnothing$ Note 4252

Decimal to Gray code

\begin{tabular}{|l|l|l||l|l||}
\hline Dec & Hex & Binary & Bits & "Hex"|“Dec" \\
\hline
\end{tabular}

\begin{tabular}{|c|c|c|c|c|c|}
\hline 129 & 81 & 10000001 & 11000001 & C1 & \\
\hline 130 & 82 & 10000010 & 11000011 & $\mathrm{C} 3$ & \\
\hline 131 & 83 & 0000011 & 11000010 & $\mathrm{C} 2$ & \\
\hline 132 & 84 & 10000100 & 1000110 & $\mathrm{C} 6$ & \\
\hline 133 & 85 & 10000101 & 11000111 & $\mathrm{C} 7$ & \\
\hline 134 & 86 & 10000110 & 101 & $\mathrm{C} 5$ & \\
\hline 135 & 87 & 10000111 & & C4 & \\
\hline 136 & 88 & 000 & & $\mathrm{CC}$ & \\
\hline 137 & 89 & 1000 & 101 & $\mathrm{CD}$ & \\
\hline 138 & $8 \mathrm{~A}$ & 1000 & 111 & $\mathrm{CF}$ & \\
\hline 139 & $8 B$ & & & $\mathrm{CE}$ & \\
\hline 140 & $8 \mathrm{C}$ & 1000 & 010 & $\mathrm{CA}$ & \\
\hline 141 & $8 D$ & 1000 & 011 & $\mathrm{CB}$ & \\
\hline 142 & $8 \mathrm{E}$ & 100 & & $\mathrm{C9}$ & \\
\hline 143 & $8 \mathrm{~F}$ & 1000 & 000 & $\mathrm{C} 8$ & \\
\hline 144 & 90 & & & $\mathrm{D} 8$ & \\
\hline 145 & 91 & 100 & & D9 & \\
\hline 146 & 92 & 100 & 111 & $\mathrm{DB}$ & \\
\hline 147 & 93 & & & $\mathrm{DA}$ & \\
\hline 148 & 94 & 100 & & $\mathrm{DE}$ & \\
\hline 149 & 95 & 100 & 111 & $\mathrm{DF}$ & \\
\hline 150 & 96 & & & $\mathrm{DD}$ & \\
\hline 151 & 97 & 100 & 100 & $\mathrm{DC}$ & \\
\hline 152 & 98 & 100 & 100 & D4 & \\
\hline 153 & 99 & & & D5 & \\
\hline 154 & $9 \mathrm{~A}$ & 100 & & D7 & \\
\hline 155 & $9 \mathrm{~B}$ & 100 & & D6 & \\
\hline 156 & $9 \mathrm{C}$ & & & D2 & \\
\hline 157 & $9 \mathrm{D}$ & 1001 & 011 & D3 & \\
\hline 158 & $9 \mathrm{E}$ & 100 & 001 & D1 & \\
\hline 159 & $9 \mathrm{~F}$ & 100 & & D0 & \\
\hline 160 & $\mathrm{AO}$ & 1010 & 000 & Fo & \\
\hline 161 & A1 & 1010 & & $\mathrm{~F} 1$ & \\
\hline 162 & $\mathrm{~A} 2$ & 10100010 & & $\mathrm{~F} 3$ & \\
\hline 163 & A3 & 1010 & 010 & $\mathrm{~F} 2$ & \\
\hline 164 & A4 & 1010 & & F6 & \\
\hline 165 & A5 & 10100101 & 11110111 & $\mathrm{~F} 7$ & \\
\hline 166 & A6 & 10100110 & 0101 & F5 & \\
\hline 167 & A7 & 10100111 & 100 & F4 & \\
\hline 168 & A8 & 10101000 & 11111100 & $\mathrm{FC}$ & \\
\hline 169 & A9 & 10101001 & 11111101 & FD & \\
\hline 170 & AA & 10101010 & 11111111 & $\mathrm{FF}$ & \\
\hline 171 & $A B$ & 10101011 & 11111110 & FE & \\
\hline 172 & $A C$ & 10101100 & 11111010 & FA & \\
\hline
\end{tabular}

\begin{tabular}{|c|c|c|c|c|c|}
\hline \multicolumn{6}{|c|}{ Gray Code to Decimal } \\
\hline Hex"|" & "Dec" & & Binary & Hex & Dec \\
\hline 129 & 81 & 10000001 & 11111110 & $\mathrm{FE}$ & \\
\hline 130 & 82 & & 11111100 & $\mathrm{FC}$ & \\
\hline 31 & 83 & 10000011 & & $\mathrm{FD}$ & 25 \\
\hline 132 & 84 & 10000100 & & $\mathrm{~F} 8$ & \\
\hline & 85 & & & $\mathrm{F9}$ & 24 \\
\hline 134 & 86 & 110 & & $\mathrm{FB}$ & 25 \\
\hline 135 & 87 & 100 & & $\mathrm{FA}$ & 25 \\
\hline 136 & 88 & & & $\mathrm{Fo}$ & 24 \\
\hline 137 & 89 & & & $\mathrm{~F} 1$ & 24 \\
\hline 138 & $8 \mathrm{~A}$ & & & $\mathrm{~F} 3$ & 24 \\
\hline & $8 B$ & & & $\mathrm{~F} 2$ & 24 \\
\hline 140 & $8 \mathrm{C}$ & 100 & & $\mathrm{~F} 7$ & \\
\hline & $8 \mathrm{D}$ & & & F6 & 24 \\
\hline 2 & $8 \mathrm{E}$ & & & $\mathrm{F} 4$ & \\
\hline 143 & $8 \mathrm{~F}$ & 10 & & F5 & 24 \\
\hline & 90 & & & E0 & 22 \\
\hline 5 & 91 & & & $\mathrm{E} 1$ & \\
\hline 146 & 92 & & & E3 & 22 \\
\hline & 93 & & & E2 & 22 \\
\hline 148 & 94 & & & E7 & 23 \\
\hline 149 & 95 & & & E6 & 23 \\
\hline & 96 & & & E4 & \\
\hline 151 & 97 & & & E5 & 22 \\
\hline & 98 & & & $\mathrm{EF}$ & 23 \\
\hline & 99 & & & EE & \\
\hline 154 & $9 \mathrm{~A}$ & & & $\mathrm{EC}$ & 2 \\
\hline 155 & $9 \mathrm{~B}$ & & & $\mathrm{ED}$ & 23 \\
\hline & $9 \mathrm{C}$ & & & E8 & \\
\hline 57 & $9 \mathrm{D}$ & 100 & & E9 & 23 \\
\hline 158 & $9 \mathrm{E}$ & & & $\mathrm{EB}$ & $\angle 0$ \\
\hline & $9 F$ & & & EA & \\
\hline 160 & $\mathrm{AO}$ & & & $\mathrm{CO}$ & 10 \\
\hline & A1 & & & $\mathrm{C} 1$ & 19 \\
\hline & $\mathrm{A} 2$ & & & $\mathrm{C} 3$ & 10 \\
\hline 163 & $\mathrm{~A} 3$ & & & $\mathrm{C} 2$ & 19 \\
\hline 164 & A4 & & & $\mathrm{C} 7$ & 19 \\
\hline & A5 & 1010 & & $\mathrm{C6}$ & 7 \\
\hline 166 & A6 & & & C4 & 19 \\
\hline & A7 & & & $\mathrm{C5}$ & 19 \\
\hline 168 & A8 & 10101000 & & $\mathrm{CF}$ & 20 \\
\hline & A9 & & & $\mathrm{CE}$ & 20 \\
\hline & $\mathrm{AA}$ & 10101010 & 11001100 & $\mathrm{CC}$ & \\
\hline & $\mathrm{AB}$ & & & $\mathrm{CD}$ & \\
\hline 172 & $\mathrm{AC}$ & 10101100 & 11001000 & C8 & 20 \\
\hline
\end{tabular}


SVX4 User's Manual

D $\varnothing$ Note 4252

Decimal to Gray code

\begin{tabular}{|l|l|l||l|l||}
\hline Dec & Hex & Binary & Bits & "Hex"|“Dec" \\
\hline
\end{tabular}

\begin{tabular}{|c|c|c|c|c|c|}
\hline 173 & $A D$ & 10101101 & & $\mathrm{FB}$ & 25 \\
\hline 174 & $\mathrm{AE}$ & 10101110 & 11111001 & F9 & \\
\hline 175 & $\mathrm{AF}$ & 10101111 & 000 & $=8$ & \\
\hline 179 & B3 & 10110011 & & $E A$ & \\
\hline 180 & B4 & 100 & & $\mathrm{EE}$ & \\
\hline 181 & B5 & 1011 & 111 & $\mathrm{EF}$ & \\
\hline 182 & B6 & 10110110 & & ED & \\
\hline 183 & B7 & & & $\mathrm{EC}$ & \\
\hline 184 & B8 & 1011 & 100 & E4 & \\
\hline 185 & B9 & & & E5 & \\
\hline 186 & BA & 010 & & E7 & \\
\hline 187 & $\mathrm{BB}$ & 101 & 110 & E6 & \\
\hline 188 & $\mathrm{BC}$ & & & E2 & \\
\hline 189 & $\mathrm{BD}$ & 101 & 011 & E3 & \\
\hline 190 & $\mathrm{BE}$ & 110 & 001 & $\mathrm{E} 1$ & \\
\hline 191 & $\mathrm{BF}$ & & & EO & \\
\hline 192 & $\mathrm{CO}$ & 000 & 000 & $\mathrm{AO}$ & \\
\hline 193 & $\mathrm{C} 1$ & & & $\mathrm{~A} 1$ & \\
\hline 194 & $\mathrm{C} 2$ & & & $\mathrm{~A} 3$ & \\
\hline 195 & $\mathrm{C} 3$ & 011 & 010 & $\mathrm{~A} 2$ & \\
\hline 196 & $\mathrm{C} 4$ & & & A6 & \\
\hline 197 & C5 & & & A7 & \\
\hline 198 & C6 & 110 & & A5 & \\
\hline 199 & C7 & & & A4 & \\
\hline 200 & $\mathrm{C} 8$ & & & $\mathrm{AC}$ & \\
\hline 201 & C9 & 001 & 101 & $A D$ & \\
\hline 202 & $\mathrm{CA}$ & & & $\mathrm{AF}$ & \\
\hline 203 & $\mathrm{CB}$ & & & $\mathrm{AE}$ & \\
\hline 204 & $\mathrm{CC}$ & 100 & & AA & \\
\hline 205 & $\mathrm{CD}$ & & & $A B$ & \\
\hline 206 & $\mathrm{CE}$ & 110 & & A9 & \\
\hline 207 & $\mathrm{CF}$ & 110 & 000 & A8 & \\
\hline 208 & Do & & & B8 & \\
\hline 209 & D1 & 11010001 & & B9 & \\
\hline 210 & D2 & 110 & & $\mathrm{BB}$ & \\
\hline 211 & D3 & & & $\mathrm{BA}$ & \\
\hline 212 & D4 & 11010100 & 1110 & $\mathrm{BE}$ & \\
\hline 213 & D5 & 110 & 1111 & $\mathrm{BF}$ & \\
\hline 214 & D6 & & & $\mathrm{BD}$ & \\
\hline 215 & D7 & 11010111 & 1100 & $\mathrm{BC}$ & \\
\hline 216 & D8 & 11011000 & 10110100 & B4 & \\
\hline 217 & D9 & & & B5 & \\
\hline 218 & DA & 11011010 & & B7 & \\
\hline 219 & $\mathrm{DB}$ & 11011011 & 10110110 & $\mathrm{Bb}$ & \\
\hline
\end{tabular}

\begin{tabular}{|c|c|c|c|c|c|}
\hline \multicolumn{6}{|c|}{ Gray Code to Decimal } \\
\hline "Hex"|" & "Dec" & Bits & Binary & Hex & Dec \\
\hline 173 & $A D$ & 10101101 & 11001001 & $\mathrm{C9}$ & 20 \\
\hline 174 & $\mathrm{AE}$ & 10101110 & 1001011 & CB & \\
\hline & $\mathrm{AF}$ & & & $\mathrm{CA}$ & 20 \\
\hline 179 & B3 & 10110011 & 101 & $\mathrm{DD}$ & 22 \\
\hline 180 & B4 & 10110100 & & $\mathrm{D} 8$ & \\
\hline 181 & B5 & & & $\mathrm{D9}$ & 217 \\
\hline 182 & $\mathrm{~B} 6$ & 10110110 & & $\mathrm{DB}$ & \\
\hline 183 & B7 & & & $\mathrm{DA}$ & \\
\hline 184 & B8 & & & $\mathrm{DO}$ & 20 \\
\hline 185 & B9 & 101 & & $\overline{D 1}$ & 20 \\
\hline 186 & $\mathrm{BA}$ & & & $\mathrm{D3}$ & \\
\hline 187 & BB & & & $\mathrm{D} 2$ & 21 \\
\hline 188 & $\mathrm{BC}$ & 101 & & D7 & \\
\hline & $\mathrm{BD}$ & & & D6 & 21 \\
\hline 190 & $\mathrm{BE}$ & & & $\mathrm{D} 4$ & 21 \\
\hline 191 & $\mathrm{BF}$ & & & D5 & 21 \\
\hline & $\mathrm{CO}$ & & & 80 & 128 \\
\hline 193 & $\mathrm{C} 1$ & & & 81 & 12 \\
\hline & $\mathrm{C} 2$ & & & 83 & 131 \\
\hline 195 & $\mathrm{C} 3$ & & & & \\
\hline 196 & C4 & & & 87 & 13 \\
\hline & $\mathrm{C} 5$ & & & 36 & 13 \\
\hline 198 & $\mathrm{C} 6$ & & & & \\
\hline 199 & C7 & & & 85 & 13 \\
\hline & $\mathrm{C} 8$ & & & & 14 \\
\hline 201 & C9 & & & & \\
\hline 202 & $\mathrm{CA}$ & & & $8 \mathrm{C}$ & 14 \\
\hline 203 & $\mathrm{CB}$ & & & OL & 14 \\
\hline 204 & $\mathrm{CC}$ & & & 88 & \\
\hline 205 & $\mathrm{CD}$ & & & 89 & 13 \\
\hline 206 & $\mathrm{CE}$ & & & & 13 \\
\hline 207 & $\mathrm{CF}$ & 110 & 100 & $8 \mathrm{~A}$ & 13 \\
\hline 208 & Do & & & $9 \mathrm{~F}$ & 15 \\
\hline & D1 & & & $9 \mathrm{E}$ & 15 \\
\hline 210 & D2 & 11010010 & & $9 \mathrm{C}$ & 15 \\
\hline 211 & D3 & & & $9 \mathrm{D}$ & 15 \\
\hline 212 & D4 & & & 98 & 15 \\
\hline 213 & D5 & 11010101 & & 99 & 15 \\
\hline 214 & D6 & & & $9 B$ & 15 \\
\hline 215 & D7 & & & $9 \mathrm{~A}$ & 15 \\
\hline 216 & D8 & 11011000 & & 90 & 14 \\
\hline 217 & D9 & & & 91 & 145 \\
\hline 218 & DA & 11011010 & 10010011 & 93 & \\
\hline 219 & $\mathrm{DB}$ & 11011011 & 10010010 & 92 & 14 \\
\hline
\end{tabular}


SVX4 User's Manual

DØ Note 4252

Decimal to Gray code

\begin{tabular}{|l|l|l||l|l||}
\hline Dec & Hex & Binary & Bits & "Hex"|“Dec" \\
\hline
\end{tabular}

\begin{tabular}{|r|r|r||r|r|r||}
\hline 220 & DC & 11011100 & 10110010 & B2 & 178 \\
\hline 221 & DD & 11011101 & 10110011 & B3 & 179 \\
\hline 222 & DE & 11011110 & 10110001 & B1 & 177 \\
\hline 223 & DF & 11011111 & 10110000 & B0 & 176 \\
\hline 224 & E0 & 11100000 & 10010000 & 90 & 144 \\
\hline 225 & E1 & 11100001 & 10010001 & 91 & 145 \\
\hline 226 & E2 & 11100010 & 10010011 & 93 & 147 \\
\hline 227 & E3 & 11100011 & 10010010 & 92 & 146 \\
\hline 228 & E4 & 11100100 & 10010110 & 96 & 150 \\
\hline 229 & E5 & 11100101 & 10010111 & 97 & 151 \\
\hline 230 & E6 & 11100110 & 10010101 & 95 & 149 \\
\hline 231 & E7 & 11100111 & 10010100 & 94 & 148 \\
\hline 232 & E8 & 11101000 & 10011100 & $9 \mathrm{C}$ & 156 \\
\hline 233 & E9 & 11101001 & 10011101 & $9 \mathrm{D}$ & 157 \\
\hline 234 & EA & 11101010 & 10011111 & $9 \mathrm{~F}$ & 159 \\
\hline 235 & EB & 11101011 & 10011110 & $9 \mathrm{E}$ & 158 \\
\hline 236 & EC & 11101100 & 10011010 & $9 \mathrm{~A}$ & 154 \\
\hline 237 & ED & 11101101 & 10011011 & $9 \mathrm{~B}$ & 155 \\
\hline 238 & EE & 11101110 & 10011001 & 99 & 153 \\
\hline 239 & EF & 11101111 & 10011000 & 98 & 152 \\
\hline 240 & F0 & 11110000 & 10001000 & 88 & 136 \\
\hline 241 & F1 & 11110001 & 10001001 & 89 & 137 \\
\hline 242 & F2 & 11110010 & 10001011 & $8 \mathrm{~B}$ & 139 \\
\hline 243 & F3 & 11110011 & 10001010 & $8 \mathrm{~A}$ & 138 \\
\hline 244 & F4 & 11110100 & 10001110 & $8 \mathrm{E}$ & 142 \\
\hline 245 & F5 & 11110101 & 10001111 & $8 \mathrm{~F}$ & 143 \\
\hline 246 & F6 & 11110110 & 10001101 & $8 \mathrm{D}$ & 141 \\
\hline 247 & F7 & 11110111 & 10001100 & $8 \mathrm{C}$ & 140 \\
\hline 248 & F8 & 11111000 & 10000100 & 84 & 132 \\
\hline 249 & F9 & 11111001 & 10000101 & 85 & 133 \\
\hline 250 & FA & 11111010 & 10000111 & 87 & 135 \\
\hline 251 & FB & 11111011 & 10000110 & 86 & 134 \\
\hline 252 & FC & 11111100 & 10000010 & 82 & 130 \\
\hline 253 & FD & 11111101 & 10000011 & 83 & 131 \\
\hline 254 & FE & 11111110 & 10000001 & 81 & 129 \\
\hline 255 & FF & 11111111 & 10000000 & 80 & 128 \\
\hline & & & & & \\
\hline
\end{tabular}

\begin{tabular}{|c|c|c|c|c|c|}
\hline \multicolumn{6}{|c|}{ Gray Code to Decimal } \\
\hline Hex" & "Dec" & & Binary & Hex & Dec \\
\hline 220 & $\mathrm{DC}$ & 11011100 & 10010111 & 97 & 15 \\
\hline 221 & DD & 11011101 & 10010110 & 96 & \\
\hline 222 & $\mathrm{DE}$ & & & 94 & 14 \\
\hline & DF & & & 95 & 14 \\
\hline 224 & E0 & & & $\mathrm{BF}$ & 19 \\
\hline 225 & E1 & & & $\mathrm{BE}$ & 19 \\
\hline 226 & E2 & 10 & & $\mathrm{BC}$ & 18 \\
\hline 227 & E3 & & & $\mathrm{BD}$ & 18 \\
\hline 228 & E4 & & & B8 & 18 \\
\hline 229 & E5 & 101 & & B9 & 185 \\
\hline & E6 & & & $\mathrm{BB}$ & 187 \\
\hline 231 & E7 & & & $\mathrm{BA}$ & 18 \\
\hline 232 & & & & Bo & 176 \\
\hline & E9 & & & 31 & \\
\hline 234 & EA & & & B3 & 17 \\
\hline 235 & EB & & & B2 & $17 \varepsilon$ \\
\hline 236 & EC & & & B7 & 18 \\
\hline 237 & ED & & & B6 & 18 \\
\hline & EE & & & 34 & 18 \\
\hline 239 & $\mathrm{EF}$ & & & B5 & \\
\hline 240 & Fo & & & $\mathrm{AO}$ & 16 \\
\hline 241 & $\mathrm{~F} 1$ & & & $\mathrm{~A} 1$ & 16 \\
\hline 242 & $\mathrm{~F} 2$ & & & A3 & 16 \\
\hline 243 & $\mathrm{~F} 3$ & 011 & & $\mathrm{~A} 2$ & 16 \\
\hline 244 & $\mathrm{~F} 4$ & & & A7 & 167 \\
\hline 245 & F5 & & & A6 & 16 \\
\hline 246 & F6 & 11110110 & & A4 & 16 \\
\hline 247 & $\mathrm{~F} 7$ & & & $\mathrm{~A} 5$ & 16 \\
\hline 248 & F8 & 11111000 & & $\mathrm{AF}$ & 17 \\
\hline 249 & F9 & 11111001 & & $\mathrm{AE}$ & 17 \\
\hline 250 & $\mathrm{FA}$ & & & $\mathrm{AC}$ & 17 \\
\hline 251 & $\mathrm{FB}$ & 11111011 & 10101101 & $A D$ & 173 \\
\hline 252 & $\mathrm{FC}$ & 11111100 & & $\mathrm{~A} 8$ & 16 \\
\hline 253 & FD & & & A9 & $16 \varsigma$ \\
\hline 254 & $\mathrm{FE}$ & 11111110 & & $\mathrm{AB}$ & 17 \\
\hline 255 & $\mathrm{FF}$ & 11111111 & 10101010 & $\mathrm{AA}$ & 17 \\
\hline
\end{tabular}




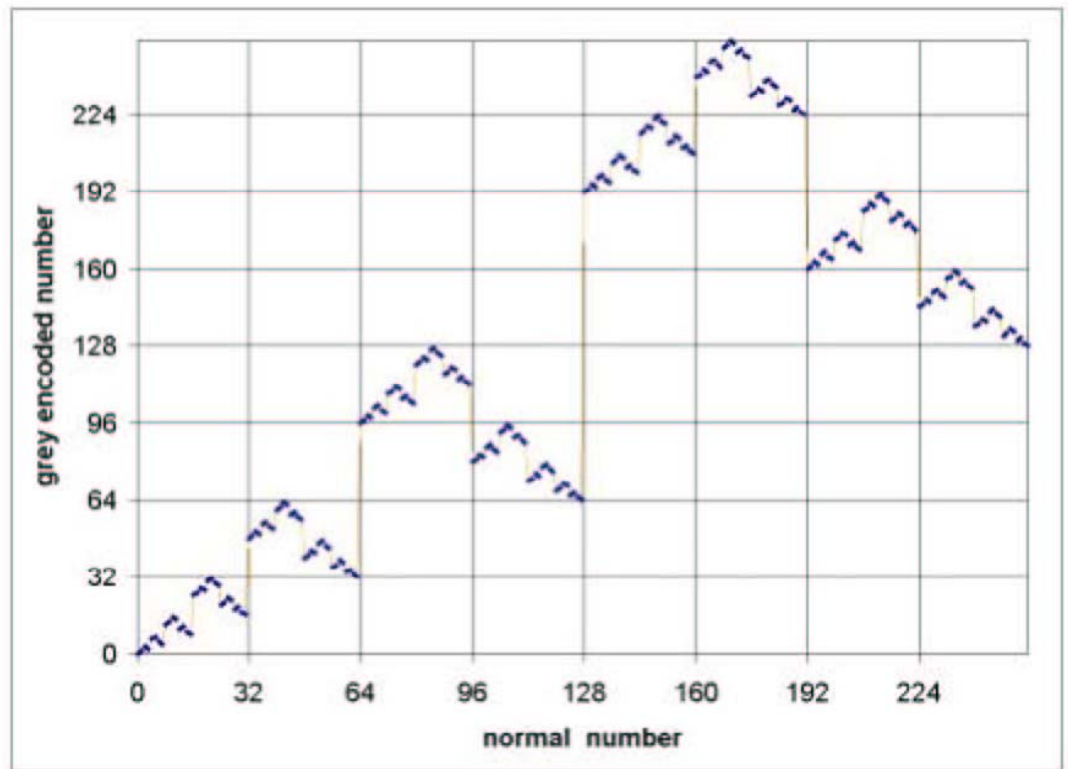

Figure 23 Gray vs. normal number. A pattern familiar to persons debugging the SVX chips, it appears for channel ID's of the SVX4 in read-all mode if one byte is missing during readout.

\footnotetext{
${ }^{1}$ More information can be found http://www-cdf.lbl.gov/users/mweber/svx4/; another repository of information can be found in the FERMI NT domain located at d0server6.fnal.gov/projects/SVX4all_docs/

${ }^{2}$ S. Kleinfelder, et. al., “A Flexible 128 Channel Silicon Strip Detector Instrumentation Integrated Circuit with Sparse Data Readout.” IEEE Transactions on Nuclear Science, Vol. 35, No. 1, February 1988, pp. 171-175.

${ }^{3}$ S. Kleinfelder, “The LBL Silicon Vertex Detector Readout Integrated Circuit: An Introduction.” Lawrence Berkeley Laboratory, November 16, 1986.
}

${ }^{4}$ T. Zimmerman, et. al., "Design of an Advanced Readout Chip for Silicon Strip Detectors.” IEEE Transactions on Nuclear Science, Vol. 40, No. 4, August 1993, pp. 736-739.

${ }^{5}$ T. Zimmerman, et. al., "SVXII Analog Pipeline Signals and Operation." Fermi National Accelerator Laboratory, April 4, 1993.

${ }^{6}$ T. Zimmerman, "SVX3: A Deadtimeless Readout Chip for Silicon Strip Detectors: The Use of Low Resistivity Substrates for Optimal Noise Reduction, Ground Referencing, and Current Conduction in Mixed Signal ASICs.” Fermilab TM, TM-2035.

${ }^{7}$ T. Zimmerman, et. al., "SVX3: A Deadtimeless Readout Chip for Silicon Strip Detectors," Nuclear Instruments and Methods in Physics Research, A 409 (1998) 369-374.

${ }^{8}$ L. Christofek et al., D $\varnothing$ Note 4251.

${ }^{9}$ L. Christofek et al., D $\varnothing$ Note 4250. 